\title{
Light Scattering in Glass Ceramic $X$-ray Imaging Plates
}

Nicola Maree Winch

\author{
A thesis \\ submitted to the Victoria University of Wellington \\ in fulfilment of the requirements for the degree of \\ Master of Science \\ in Physics
}

Victoria University of Wellington 2008 



\begin{abstract}
Glass ceramic materials have been suggested as a possible high resolution replacement for current commercial storage phosphor imaging plates. The low spatial frequency of the current plates is caused by strong scattering of the laser light incident on the plate during the read-out process. Glass ceramic materials show very small scattering due to their transparent nature, which should lead to a higher resolution. However, a competing argument is the small amount of scattering that does occur travels a much greater distance in the plate, limiting the resolution. The aim of this thesis was to simulate the scattering of light in imaging plates and use this to optimise the trade-off between resolution, sensitivity and transparency which is implicit in plate design. Additionally, experiments were performed to determine the resolution of glass ceramic and commercial imaging plates. Simulations show that high resolution can be achieved in both the strong and weak scattering limits, corresponding to opaque and transparent materials. Increasing the absorption of the laser light increases the resolution, as does decreasing the laser beam diameter and power. An increase in the resolution almost always comes at a cost of a decrease in the sensitivity. The resolutions of an Agfa MD30 and glass ceramic imaging plate were found to be 4.5 line pairs $/ \mathrm{mm}$ and $6.5-8.0$ line pairs/mm respectively for an MTF equal to 0.2 .
\end{abstract}





\section{Acknowledgements}

I would firstly like to thank my supervisor Associate Professor Andy Edgar for all of the support and guidance he has given me.

I am grateful for assistance from the technical staff in the School of Chemical and Physical Sciences at Victoria University, in particular Alan Rennie and Manu Pouajen-Blackiston for the manufacture of mechanical equipment as well as Johnny McClymont and Jason Edwards for their support with electronic equipment.

I am grateful to Chris Varoy for his help with sample preparation, and to Professor Heinz von Seggern for some interesting discussions.

Thank you to Dr P. J. Marsh from Monash University, Melbourne for the fluorochlorozirconate glass sample used in this work.

I would like to thank the students at Victoria University for all their help, particularly Dmitri Schebarchov, Matthias Meyer and Ben Drayton for assistance with simulation and LabVIEW code.

I acknowledge financial support from the Foundation for Research, Science and Technology account VicX0402, Victoria University, as well as the Ernest Marsden Scholarship in Physics which I received in 2007.

Finally I would like to thank all my friends and family for their continual support and encouragement. 



\section{Contents}

1 Introduction: X-ray Imaging 1

2 Theoretical Background and Review of Literature $\quad 7$

2.1 Glass ceramics and the photostimulated luminescence effect . . 7

2.2 Light scattering $\ldots \ldots \ldots \ldots \ldots$

2.2.1 Rayleigh scattering . . . . . . . . . . . . 10

2.2 .2 Mie theory . . . . . . . . . . . . . 12

2.2.3 Henyey-Greenstein approximation . . . . . . . . . . . 15

2.3 Reflection . . . . . . . . . . . . . . . . 16

2.4 Modulation transfer function . . . . . . . . . . . . . . 18

2.5 Literature review . . . . . . . . . . . . . . . . . . 20

3 Scattering Simulations $\quad 25$

3.1 Monte Carlo methods . . . . . . . . . . . . . . . . . . . . . 25

3.2 Random number generation . . . . . . . . . . . . . . 26

3.3 Overview of the model . . . . . . . . . . . . . . . 27

3.4 Photon packet description of the laser beam . . . . . . . . . 28

3.5 Simulation of the scattering process $\ldots \ldots 30$

3.6 Absorption of photons ................. 34

3.7 Reflection from surfaces . . . . . . . . . . . . . 34

3.8 Intensity distribution profiles $\ldots \ldots \ldots . \ldots \ldots$

3.9 Probability of escape . . . . . . . . . . . . . 35

3.10 Line spread and modulation transfer functions . . . . . . . . 37 
4 Scattering Simulation Results $\quad 41$

4.1 Introduction . . . . . . . . . . . . . . . 41

4.2 Dependence on scattering length . . . . . . . . . . . 42

4.3 Variations in absorption coefficient . . . . . . . . . . . 48

4.4 Adding selective absorption of the stimulating light . . . . . . . 51

4.5 Effect of particle size . . . . . . . . . . . . . . 54

4.6 Effect of surface reflection . . . . . . . . . . . . 57

4.7 Variations in laser power . . . . . . . . . . . . . . 61

4.8 Effect of laser beam variance . . . . . . . . . . . . . . . 63

4.9 Dependence on x-ray attenuation coefficient $\ldots . . \ldots 66$

5 Experimental Techniques $\quad 71$

5.1 Glass ceramic sample preparation . . . . . . . . . . . . . . 71

5.2 Absorption and scattering ................. 72

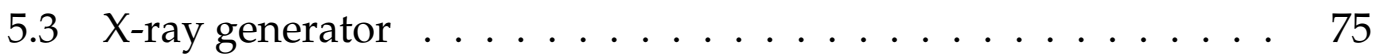

5.4 MTF measurements . . . . . . . . . . . . . . 76

$5.4 .1 \mathrm{XYZ}$ translation stage $\ldots \ldots \ldots \ldots 78$

5.4 .2 Computer interface . . . . . . . . . . . . 80

5.4 .3 LabVIEW program ................. 81

6 Experimental Results $\quad 85$

6.1 Read-out laser . . . . . . . . . . . . . . . 85

6.2 Agfa MD30 imaging plate . . . . . . . . . . . . . 87

$6.3 \mathrm{~N} 05-02-50$ glass ceramic $\ldots \ldots . \ldots . \ldots . \ldots 92$

$\begin{array}{lll}7 & \text { Discussion and Conclusions } & 97\end{array}$

$\begin{array}{ll}\text { Appendix A } & 103\end{array}$

$\begin{array}{ll}\text { References } & 105\end{array}$ 


\section{List of Figures}

1.1 X-ray attenuation coefficient versus energy for various materials 2

1.2 Process of photostimulated luminescence . . . . . . . . . . 3

1.3 The imaging process using storage phosphor imaging plates . . 4

2.1 PSL efficiencies, transmittance and visual appearance of annealed glass ceramics ....................... 8

2.2 Rayleigh phase function . . . . . . . . . . . . . . . . 12

2.3 Mie phase function . . . . . . . . . . . . . . . . 14

2.4 Henyey-Greenstein phase function . . . . . . . . . . . . 16

2.5 Reflected and refracted waves at a boundary . . . . . . . . 17

2.6 Determination of the MTF by imaging a lead grid. . . . . . . . 19

2.7 Determination of the MTF by imaging a sharp edge. . . . . . . 20

2.8 MTF for a glass ceramic and Agfa MD30 imaging plate . . . . . 22

3.1 Quadrant of circle in box for Monte Carlo determination of $\pi$. . 25

3.2 Probability density and distribution functions . . . . . . . 29

3.3 Simulated laser beam incident on plate for a beam variance of

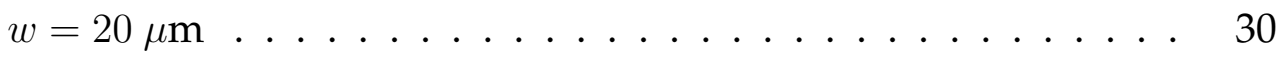

3.4 Scattering of photon packets within the plate . . . . . . . . 31

3.5 Euler angle geometry . . . . . . . . . . . . . . . 33

3.6 Scattering profiles of the incident read-out laser after following a set number of packets through the plate . . . . . . . 35

3.7 Conceptual picture showing how the LSF is determined . . . . . 37 
4.1 Intensity distributions profiles for large particles with varying scattering lengths . . . . . . . . . . . . . . 43

4.2 Probability of escape from front surface for various scattering

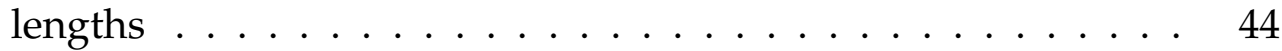

4.3 LSF's and MTF's for photons escaping from the front surface with various scattering lengths $\ldots \ldots \ldots \ldots$

4.4 Sensitivity and resolution for photons escaping from the front surface with various scattering lengths $\ldots \ldots \ldots$

4.5 Probability of escape from rear surface for various scattering

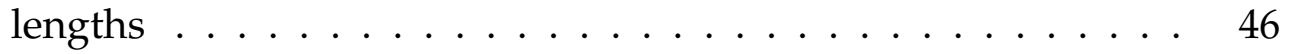

4.6 LSF's and MTF's for photons escaping from the rear surface with various scattering lengths $\ldots \ldots \ldots \ldots$

4.7 Sensitivity and resolution for photons escaping from the rear surface with various scattering lengths $\ldots \ldots \ldots \ldots$

4.8 Intensity distribution profiles for varying absorption coefficients

4.9 Probability of escape from the front surface for various absorption coefficients ...................... 50

4.10 LSF's and MTF's for photons escaping from the front surface with various absorption coefficients . . . . . . . . . . 50

4.11 Sensitivity and resolution for photons escaping from the front surface with various absorption coefficients . . . . . . . . 51

4.12 LSF's and MTF's for photons escaping from the front surface with various laser photon absorption coefficients . . . . . . . .

4.13 Reproduced LSF's and MTF's for photons escaping from the front surface with various absorption coefficients . . . . . . . . 53

4.14 Sensitivity and resolution for photons escaping from the front surface with various laser photon absorption coefficients . . . 53

4.15 Intensity distribution profiles for various particle sizes . . . . 55 
4.16 Probability of escape from the front surface for various particle

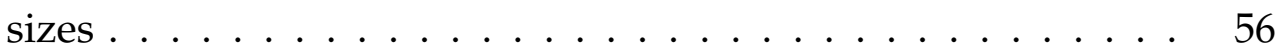

4.17 LSF's and MTF's for photons escaping from the front surface with various particle sizes . . . . . . . . . . 56

4.18 Sensitivity and resolution for photons escaping from the front surface with various particle sizes . . . . . . . . . . 57

4.19 Intensity distribution profiles for the cases of reflection and no reflection from surfaces . . . . . . . . . . . . . . . 58

4.20 Probability of escape from front surface including and not including reflection from surfaces $\ldots \ldots \ldots \ldots$

4.21 LSF's and MTF's for photons escaping from the front surface for the cases of reflection and no reflection $\ldots \ldots \ldots$

4.22 LSF's and MTF's for photons escaping from the front surface with various laser powers . . . . . . . . . . . . 62

4.23 Variations in storage centre density with different laser powers 62

4.24 Sensitivity and resolution for photons escaping from the front surface with various laser powers . . . . . . . . . . . . 63

4.25 Intensity distribution profiles for various laser variances . . . . 64

4.26 LSF's and MTF's for photons escaping from the front surface with various laser variances . . . . . . . . . . . . . 65

4.27 Sensitivity and resolution for photons escaping from the front surface with various laser variances $\ldots \ldots$. . . . . . . 65

4.28 Intensity distribution profiles for scattering lengths of $20 \mu \mathrm{m}$ and $2000 \mu \mathrm{m} \ldots \ldots \ldots \ldots \ldots \ldots \ldots$

4.29 LSF's and MTF's for photons escaping from the front surface with $\mu=20 \mu \mathrm{m}$ and various $x$-ray energies $\ldots \ldots . \ldots 68$

4.30 Sensitivity and resolution for photons escaping from the from the front surface with various $x$-ray energies $\ldots \ldots \ldots$ 
4.31 LSF's and MTF's for photons escaping from the front surface with $\mu=2000 \mu \mathrm{m}$ and various $x$-ray energies $\ldots \ldots \ldots 70$

5.1 DSC measurements of a typical glass ceramic sample . . . . . 72

5.2 Optical schematic of Shimadzu UV-Vis 2100 spectrophotometer 73

5.3 Czerny-Turner monochromator design . . . . . . . . . . . 73

5.4 Diagram of Faxitron x-ray tube . . . . . . . . . . . 75

5.5 Schematic of experimental image read-out system . . . . . . . 77

5.6 Experimental image read-out system . . . . . . . . . . . 78

5.7 Converting rotational motion into translational motion . . . . 79

5.8 Circuit diagram of cables for SuperLogics ADC-1 box . . . . . . 81

5.9 LabVIEW block diagram for controlling stepping of the motors and the home switch . . . . . . . . . . . . 83

5.10 LabVIEW block diagram for controlling the scanning program . 84

6.1 Image of gold grid obtained by scanning laser across the grid and recording the reflected red light $\ldots \ldots \ldots$

6.2 Image of sharpest edge of gold grid and profile of the laser spot 86

6.3 Two consecutive scans of an edge stored on an Agfa MD30 imaging plate ......................... 87

6.4 Structure of the Agfa MD4.0 general plate . . . . . . . . . . . 88

6.5 Scattering length versus wavelength for Agfa MD30 . . . . . . . 89

6.6 SEM images of cross-section of Agfa MD30 imaging plate . . . . 90

6.7 Image of lead grid read-out from Agfa MD30 . . . . . . . . . . . 90

6.8 ESF and MTF for Agfa MD30 imaging plate . . . . . . . . . . . . 91

6.9 Scattering length versus wavelength for the various crystallisation regions of N05-02-50 glass ceramic sample . . . . . . . . 93

6.10 XRD pattern of the N05-02-50 glass ceramic sample $\ldots . . . .94$

6.11 Image of gold grid produced by N05-02-50 glass ceramic . . . . 94

6.12 ESF and MTF for N05-02-50 glass ceramic sample . . . . . . . 95 
6.13 MTF's for both the glass ceramic sample and Agfa MD30 imag-

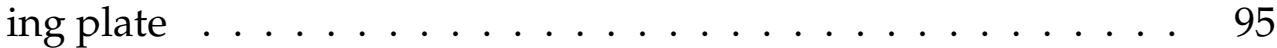





\section{List of Tables}

4.1 Simulation parameters held constant in all simulations . . . . . 42

4.2 Parameters for simulations with varying scattering lengths . . . 42

4.3 Parameters for simulations with varying absorption coefficients 48

4.4 Parameters for simulations with added absorption . . . . . . . 52

4.5 Parameters for simulations with various particle sizes . . . . . . 54

4.6 Anisotropy coefficients and size parameters for various particle radii ................................. 54

4.7 Parameters for simulations for the cases of reflection or no reflection from surfaces. . . . . . . . . . . . . . . 58

4.8 Parameters for simulations with varying laser powers . . . . . 61

4.9 Parameters for simulations with varying laser variances . . . . . 63

4.10 Parameters for simulations with varying $x$-ray attenuation coef-

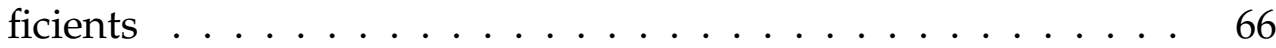

4.11 Relationship between x-ray energy, attenuation coefficient and half length ......................... 66

5.1 Half step phase pattern . . . . . . . . . . . . . . 79

5.2 Commands for SuperLogics ADC-1 . . . . . . . . . . 80 



\section{Chapter 1}

\section{Introduction: X-ray Imaging}

Ever since $\mathrm{x}$-rays were discovered in 1895 they have been valuable in applications such as medical diagnosis, non destructive testing and airport security. X-rays are electromagnetic waves with shorter wavelength and higher energy than visible light and are produced by accelerating electrons towards a metal target. These electrons decelerate on collision with the target and if they contain enough energy, are able to knock an electron from the inner shell of the atom. Consequently, electrons from higher energy states fill up the vacancy, emitting $\mathrm{x}$-ray photons in the process.

A shadow image of an object can be created with $\mathrm{x}$-rays through the processes of the photoelectric effect and Compton scattering. The attenuation of $x$-rays by a material depends on the composition, thickness, density and atomic number $(Z)$. Materials with a high atomic number, such as lead, attenuate $\mathrm{x}$-rays more strongly than materials with a lower atomic number, such as human tissue. This can be seen in figure 1.1 where the attenuation coefficient for different materials is plotted against $x$-ray energy. At low $x$-ray energies the attenuation is dominated by the photoelectric effect which has a $Z^{4}$ dependence on the atomic number. At high $\mathrm{x}$-ray energies the attenuation coefficient follows the linear $Z$ dependence of Compton scattering. Steps in the attenuation coefficient versus energy graph are due to core level transitions in the atoms which make up the material. 


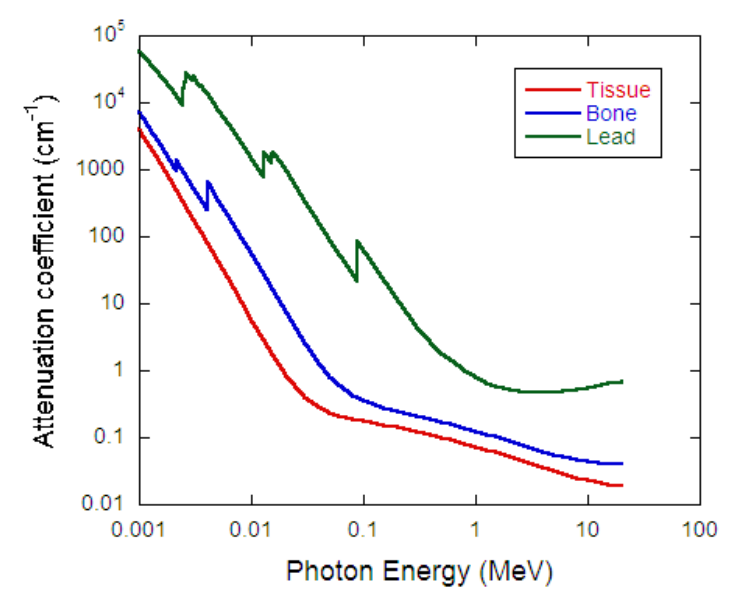

Figure 1.1: X-ray attenuation coefficient versus energy for various materials.

The first technique of $x$-ray imaging was based on photographic film. X-ray film is made by coating a plastic base with a thin layer of light sensitive emulsion which is composed of a silver halide mixed with a gelatine. Photons which are incident on the halide grains produce photogenerated free electrons which interact with the silver ions to produce silver metal atoms. The higher the number of photons landing on the grains the larger the concentration of silver atoms which cluster to form silver particles. The image is represented by the varying concentration of silver particles. After exposure the film is then chemically processed in order to enhance the image further ("development") and to preserve it ("fixing"). The grain size of the silver halide atoms determines the resolution and sensitivity of the film, and the maximum dose is limited by the number of silver halide grains.

However, the film is not particularly sensitive to x-rays; instead it is much more sensitive to visible light. To exploit this, intensifying screens made from materials such as calcium tungstate or rare-earth phosphors, are sandwiched with the x-ray film. The intensifying screen absorbs the incident x-rays and converts them into visible light through a process known as x-ray induced luminescence. Adding an intensifying screen increases the sensitivity of the system but the high spatial resolution is reduced due to light scattering within the screen. 


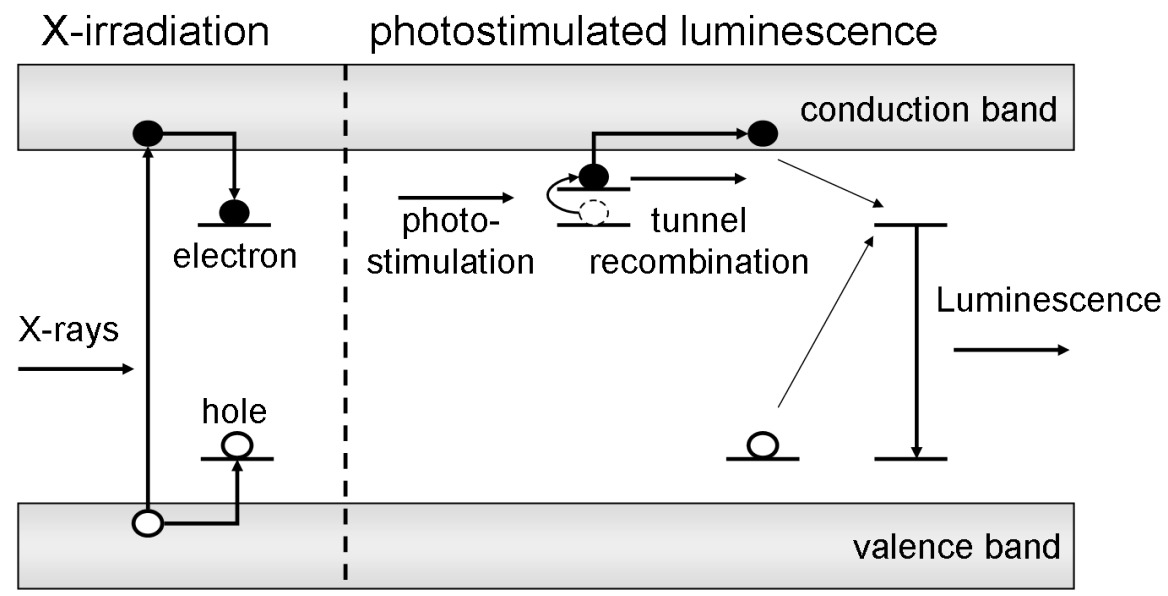

Figure 1.2: Process of photostimulated luminescence.

X-ray storage phosphor (XRSP) imaging plates were introduced in 1983 by Sonda et al [1] as an alternative to x-ray film systems. Current commercial imaging plates mainly consist of a support film covered with an organic binder in which BaBrF: $\mathrm{Eu}^{2+}$ crystals are embedded. The mechanism by which storage phosphors can be used as imaging plates is known as photostimulated luminescence (PSL) and is illustrated in figure 1.2.

X-rays which are incident on the imaging plate during irradiation cause electrons to be promoted into the conduction band in the BaFBr:Eu crystals. Instead of promptly recombining with the corresponding holes, some of these electrons are trapped just below the conduction band in electron traps. These traps are commonly anion vacancies, which when occupied by a trapped electron are known as F centres [2]. Similarly the holes are trapped just above the valence band in hole traps, for example $\mathrm{V}_{\mathrm{k}}$ centres [2]. The latent image in the plate is then stored as a spatial variation in the concentration of trapped electrons and holes; the concentration is proportional to the x-ray dose.

During the read-out process, a focussed red $(\sim 630 \mathrm{~nm})$ laser beam is raster scanned across the plate. The energy of the light is high enough that the electrons or holes are able to overcome their trap barriers. Recombination of the electrons and holes then occurs with the recombination energy being transferred to the $\mathrm{Eu}^{2+}$ ions. The $\mathrm{Eu}^{2+}$ ions enter an excited state and then decay 


\section{X-ray exposure}
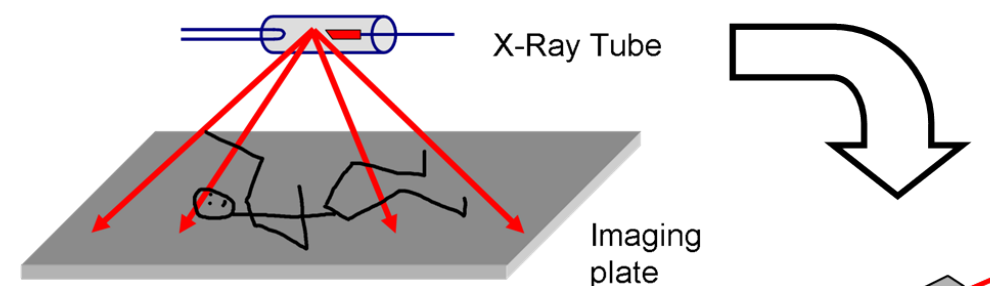

\section{Image readout}
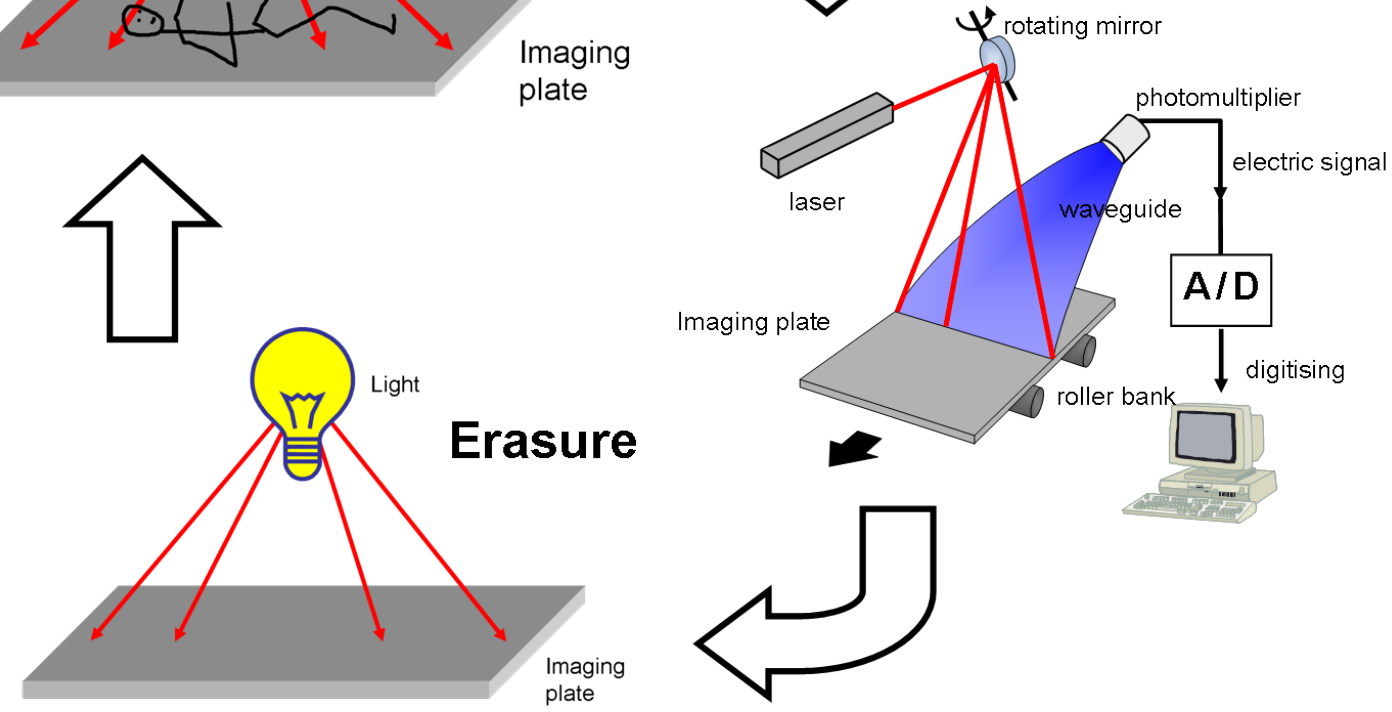

Figure 1.3: The imaging process using storage phosphor imaging plates.

after a short period of time, emitting characteristic luminescence at $\sim 390 \mathrm{~nm}$, which is separated from the red laser light by appropriate filtration.

The imaging process for XRSP plates is illustrated in figure 1.3. The emitted photons are directed by a fibre optic cable to a photomultiplier. The intensity of the emitted light and the photomultiplier signal is proportional to the density of trapped electron-hole pairs. The photomultiplier converts the luminescence into an electrical signal which is passed through an A/D converter into a computer for reconstruction of the image. Once the image has been read-out, the plate is bleached (all remaining traps emptied) so the imaging plate can be reused.

Storage phosphor imaging plates have many advantages over conventional film methods, such as:

- High sensitivity - a lower dose can be applied - greater patient safety.

- Large dynamic range - around 5 orders of magnitude, greater than the $2-3$ orders of magnitude for film. This prevents under and over exposed 
images and the corresponding necessity for re-takes.

- Reusable - plates are bleached after each use - up to 1,000 to 10,000 exposures.

- Direct digital image - advantageous for image processing/enhancements and tele medicine.

- No chemical processing - convenient and safer for radiologist staff.

The main disadvantage in these imaging plates is the poor spatial resolution due to scattering of the read-out laser.

During the read-out process, the laser light which is incident on the storage phosphor is strongly scattered off the crystallites. This scattering causes the stimulated area to be greater than the area of the laser beam, which in turn causes the spatial resolution to be limited to around 2.5 line pairs $/ \mathrm{mm}(\mathrm{lp} / \mathrm{mm})$. X-ray film has a spatial resolution of approximately $5 \mathrm{lp} / \mathrm{mm}$, which means $\mathrm{x}$ ray film is still used for high resolution applications such as mammography (this application requires high resolution for early detection and diagnosis of treatable illnesses such as breast cancer).

To overcome the resolution problem, a recent approach has been to examine transparent glass ceramics as storage phosphors [3]. These glass ceramics are typically fluorozirconate glasses co-doped with bromine or chlorine and europium. The glass ceramics have an advantage in that they are semitransparent and so less scattering should occur. In addition, glasses can be moulded into a variety of shapes meaning new types of imaging systems could be designed, for example based on fibre optics.

Other types of digital imaging systems are being developed which are rivals for the storage phosphor imaging plate. These include methods such as those based on photodiode arrays, and a phosphor screen linked to a CCD array. Most of the new digital systems require a separate reader for each individual detector with replacement costs for each detector being very high. Storage 
phosphors have an advantage over these systems as one reader can support multiple plates and the existing $\mathrm{x}$-ray sets can be retained, simply by replacing existing screen/film cassettes with imaging plate cassettes.

The ability of a near-transparent XRSP to be used for high resolution imaging is currently under debate. On one hand the scattering is weaker which at first suggests better resolution; on the other hand, what scattering there is permeates greater distances from the point of irradiation, implying a lower resolution.

The aim of this thesis is to simulate the scattering of the read-out laser light to discover how the variation in changeable variables, such as scattering length and absorption affects the scattering and resolution. In particular, the trade-off between transparency, sensitivity and resolution which can be achieved in the production of imaging plates is of prime interest. Simulation results can then be applied in refining the materials design of high resolution glass ceramic imaging plates.

The thesis is structured as follows. Chapter 2 contains background information on scattering and related theory, and a brief review of relevant earlier work reported in the literature. Chapter 3 describes how the scattering simulations were formulated. Chapter 4 contains results of the simulations for a spectra of possible imaging plate properties. Chapter 5 describes the experimental methods used, and Chapter 6 contains experimental results for commercial and glass-ceramic XRSP plates and compares them with experimental simulations. 


\section{Chapter 2}

\section{Theoretical Background and}

\section{Review of Literature}

\subsection{Glass ceramics and the photostimulated lumi- nescence effect}

Photostimulated luminescence has been observed in simple glasses, for example in rare earth doped alkali borate and fluoroaluminate glasses by Qui et al in 1997 ([4] [5] [6]), although the PSL intensity was not reported. Subsequent measurements [7] suggested that only a very small effect of negligible commercial significance could be observed.

Glass ceramics are materials comprised of nano or micro-crystallites embedded in a glass matrix. The material is first made as a glass by quenching from the melt and then heated to induce crystallite growth. The glass matrix commonly consists of a mix of alkali and silicon, phosphorous, boron or aluminium oxides; the crystals are of various compositions but share the properties of being insoluble at low temperatures in the glass.

Fluorozirconate glasses are heavy-metal fluoride glasses based on the glassforming properties of zirconium fluoride. One member of this family is ZBLAN glass which is composed of fluorides of zirconium, barium, lanthanum, alu- 


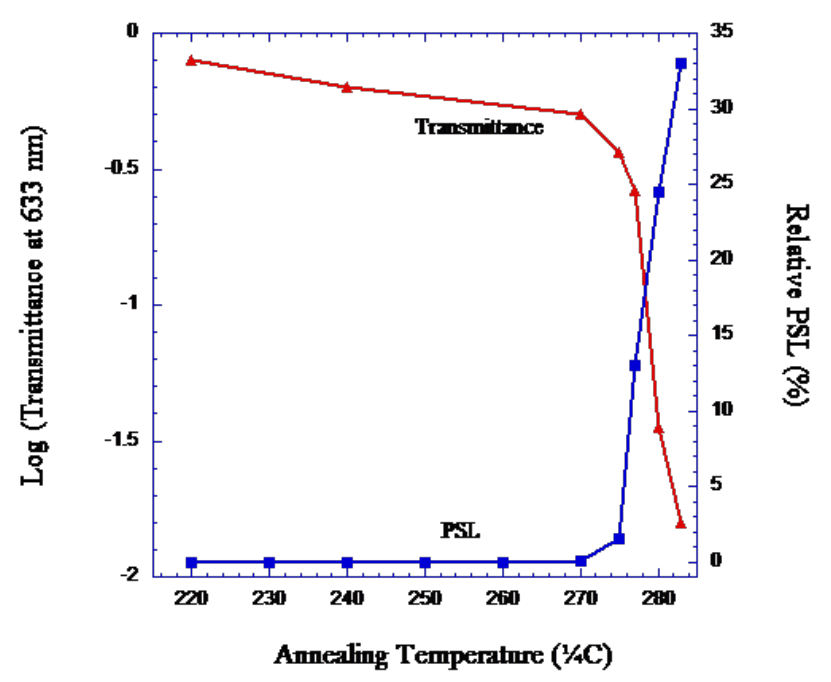

(a)

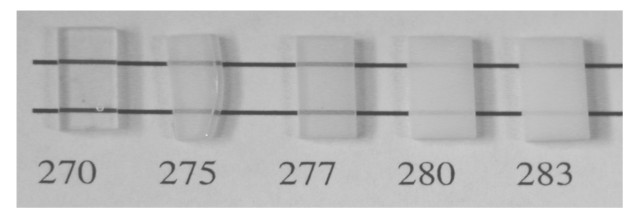

(b)

Figure 2.1: (a) PSL efficiencies relative to $\mathrm{BaFBr}: \mathrm{Eu}^{2+}$ and transmittance for annealed glass. The lines are guides to the eye. (b) Visual appearance of glasses annealed at temperatures $\left({ }^{\circ} \mathrm{C}\right)$ as indicated. [9]

minium and sodium. Compared to more traditional glasses, ZBLAN has a higher refractive index and transmits further into the infrared which makes it useful for optical fibres in communications and medical applications.

By replacing $\mathrm{F}^{-}$ions in a fluorozirconate glass with $\mathrm{Br}^{-}$ions, Edgar et al [3] observed a significant PSL effect. However, the PSL conversion efficiencies (the fraction of x-ray energy converted to PSL light) was around $0.025-0.05 \%$ that of the standard XRSP material BaFBr:Eu${ }^{2+}$. Later, Schweizer et al [8] found by replacing the $\mathrm{Br}^{-}$ions with $\mathrm{Cl}^{-}$ions a larger PSL signal is achieved with a conversion efficiency of up to $80 \%$ that of $\mathrm{BaFBr}_{\mathrm{Eu}}{ }^{2+}$. When first made these fluorozirconate glasses show no PSL effect and are transparent. The glasses are then annealed (heat treated) to induce growth of $\mathrm{BaCl}_{2}$ crystallites. Initially $\mathrm{BaCl}_{2}$ forms in a hexagonal phase and as the annealing temperature is increased the orthorhombic phase of $\mathrm{BaCl}_{2}$ is seen [9]. The orthorhombic phase shows the strongest PSL effect. 
Once the temperature for orthorhombic phase $\mathrm{BaCl}_{2}$ crystallites has been reached any further increase in temperature causes the crystallites to grow in size rather than number. This affects both the PSL and transparency of the glass. As can be seen in figure 2.1(a) higher annealing temperatures produce opaque glass ceramics which show a strong PSL intensity. Glasses annealed at a lower temperature show a weak PSL intensity but are relatively transparent. The visual appearance of the glass ceramics for different annealing temperatures is shown in figure 2.1(b).

One of the key aims of this thesis is to provide guidance on the thermal processing for optimisation of the imaging performance of these glass ceramic materials.

\subsection{Light scattering}

Scattering of an electromagnetic (EM) wave can arise from structural inhomogeneities in a medium and the corresponding spatially varying refractive index. The scattering of light together with selective absorption explains why objects have particular colours under illumination by white light.

Matter is composed of discrete electric charges - electrons and protons. If an obstacle in the material is illuminated by an EM wave it sets the electric charges into oscillatory motion due to this incident wave. These oscillating particles emit EM radiation in all directions - a secondary radiation which is known as scattered radiation. If instead these particles change the incident radiation into some other form (for example heat) the process is known as absorption.

The effects of scattering are different depending on the concentration of scattering centres involved - either single scattering off one centre or multiple scattering involving many centres. Multiple scattering is a coherent phenomenon and can be difficult to deal with especially if the interaction between scattering centres must be taken into account. Scattering can be elastic or inelastic 
depending on whether the energy is transferred during scattering. Rayleigh and Mie scattering are examples of elastic scattering where there is no energy transfer during scattering. In contrast, Compton scattering is a type of x-ray inelastic scattering where the energy of an x-ray photon decreases after it interacts with matter (details can be found for example in [10]). The interaction between electrons in the material with a high energy photon (x-ray) results in the ejection of an electron from an atom or ion which carries away part of the incident photon energy; a photon with the remaining energy is emitted in a different direction to the incident photon, consistent with the conservation of momentum for the incident photon, emitted x-ray photon and the electron. For lower energy $\mathrm{x}$-ray photons, all of the photon energy may be transferred to the ejected electron; in this case the effect is known as the photoelectric effect.

\subsubsection{Rayleigh scattering}

Rayleigh scattering (after Lord Rayleigh 1871) describes the scattering of light by dielectric spherical particles which are much smaller than the wavelength of the radiation.

The size of the scattering particle is defined by the size parameter $x$ (equation 2.1) where $a$ is the radius of the sphere, $\lambda$ is the wavelength of incident radiation and $n_{\mathrm{m}}$ is the refractive index of the medium.

$$
x=\frac{2 \pi a n_{\mathrm{m}}}{\lambda}
$$

The Rayleigh scattering regime corresponds to the size parameter being much less than one.

Since the scatterer is very small compared to the wavelength in this regime, the electric field is assumed to be uniform over this small distance. The scatterer is then modeled by assuming it behaves like a dipole. Incident EM radiation on 
the scatterer induces the dipole, causing it to oscillate and radiate. By considering the total electric field inside the scatterer, results such as the cross-section and phase function can be found. Detailed analysis of Rayleigh scattering can be found for example in Kerker [11].

The differential cross-section is defined as the power radiated in the scattered direction per unit solid angle, per unit incident flux and is given in equation 2.2 where $n$ is the relative refractive index $n_{\mathrm{p}} / n_{\mathrm{m}}, n_{\mathrm{p}}$ is the refractive index of the particle and $\theta$ is the angle of scattering.

$$
\frac{d \sigma}{d \Omega}=\frac{a^{6}}{2} n_{\mathrm{m}}^{4}\left(\frac{2 \pi}{\lambda}\right)^{4}\left(\frac{n^{2}-1}{n^{2}+2}\right)^{2}\left(1+\cos ^{2} \theta\right)
$$

The scattering cross-section, $\sigma_{s c a}$ is the effective area which removes the incident radiation from the forwards direction and is found by integrating the differential cross-section over the sphere surrounding the scatterer, to give,

$$
\sigma_{\mathrm{sca}}=\frac{128 \pi^{5}}{3} \frac{a^{6}}{\lambda^{4}} n_{\mathrm{m}}^{4}\left(\frac{n^{2}-1}{n^{2}+2}\right)^{2}
$$

Rayleigh scattering is commonly seen in gases (where the refractive index of the medium is almost equal to one) and the scattering cross-section is often quoted in the form of equation 2.4 by assuming $n_{\mathrm{m}}=1$.

$$
\sigma_{\mathrm{sca}}=\frac{128 \pi^{5}}{3} \frac{a^{6}}{\lambda^{4}}\left(\frac{n_{\mathrm{p}}^{2}-1}{n_{\mathrm{p}}^{2}+2}\right)^{2}
$$

In the cross-section equations there is a $\lambda^{-4}$ dependence which explains why the sky is blue. Gas atoms in the atmosphere scatter the light which is incident from the sun. Blue light is predominately scattered because of its shorter wavelength leading to the sky being seen as blue, whilst the setting sun appears red as blue light is scattered out of the direct beam.

When light is scattered, the angle at which it scatters is a function of the properties of the particle and material. The phase function describes the scattering 


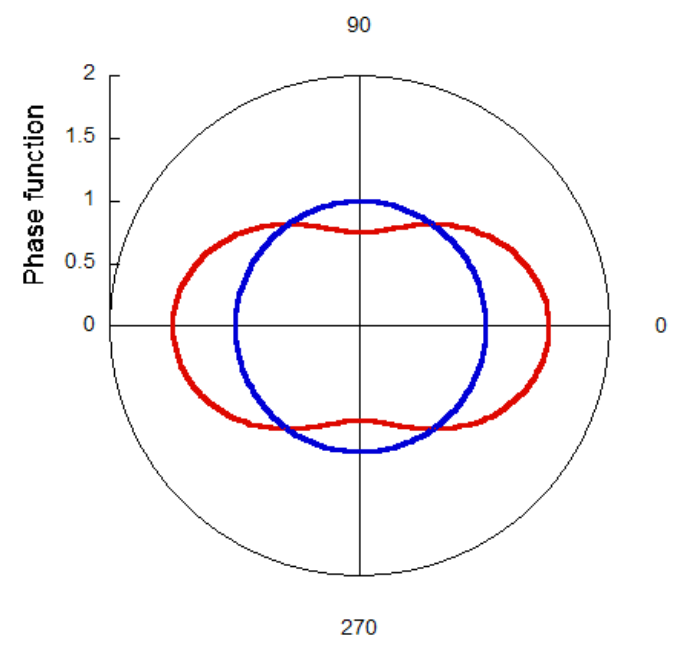

Figure 2.2: The phase function for Rayleigh scattering (red curve) and isotropic scattering (blue curve).

intensity as a function of the angle of scattering and is found by dividing the total differential cross-section (equation 2.2) by the cross-section (equation 2.3) and normalising by $4 \pi$. The Rayleigh phase function is given in equation 2.5 and shown graphically in figure 2.2.

$$
p_{\mathrm{R}}(\theta)=\frac{3}{4}\left(1+\cos ^{2} \theta\right)
$$

\subsubsection{Mie theory}

Mie theory describes the scattering of radiation by spherical particles and is an analytical solution to Maxwell's equations inside and outside the particle, matched at the boundary. It is a complete theory which covers all particle sizes and refractive indices, both real and complex. A complete explanation of Mie theory can be found in Bohren and Huffman [12].

Maxwell's equations are used to derive a wave equation for incident and scattered electromagnetic radiation in three dimensions with appropriate boundary conditions at the surface of the particle. Due to spherical symmetry the wave equation is expressed in spherical polar coordinates $(r, \theta, \phi)$. The wave 
equation is a separable partial differential equation, the solution of which is expressed as an infinite series of functions containing sines and cosines, spherical Bessel functions $\left(\psi_{m}(x), \xi_{m}(x)\right)$ and Legendre polynomials $\left(P_{m}^{1}(\cos \theta)\right)$.

The main equations required to generate a solution for a given system are the scattering coefficients, $a_{m}$ and $b_{m}$ (equation 2.6), and the angle-dependent functions $\pi_{m}$ and $\tau_{m}$ (equation 2.7).

$$
\begin{gathered}
a_{m}=\frac{n \psi_{m}(n x) \psi_{m}^{\prime}(x)-\psi_{m}(x) \psi_{m}^{\prime}(n x)}{n \psi_{m}(n x) \xi_{m}^{\prime}(x)-\xi_{m}(x) \psi_{m}^{\prime}(n x)} \\
b_{m}=\frac{\psi_{m}(n x) \psi_{m}^{\prime}(x)-n \psi_{m}(x) \psi_{m}^{\prime}(n x)}{\psi_{m}(n x) \xi_{m}^{\prime}(x)-n \xi_{m}(x) \psi_{m}^{\prime}(n x)} \\
\pi_{m}=\frac{P_{m}^{1}(\cos \theta)}{\sin \theta} \\
\tau_{m}=\frac{d P_{m}^{1}(\cos \theta)}{d \theta}
\end{gathered}
$$

The scattering and extinction cross-sections (equation 2.8 and 2.9) are equations involving infinite sums of the scattering coefficients and the wavenumber $k$. The extinction cross-section is defined as the effective area of a totally absorbing material that would absorb the same amount of radiation as the particle.

$$
\begin{gathered}
\sigma_{\text {sca }}=\frac{2 \pi}{k^{2}} \sum_{m=1}^{\infty}(2 m+1)\left(\left|a_{m}\right|^{2}+\left|b_{m}\right|^{2}\right) \\
\sigma_{\text {ext }}=\frac{2 \pi}{k^{2}} \sum_{m=1}^{\infty}(2 m+1) \operatorname{Re}\left(a_{m}+b_{m}\right)
\end{gathered}
$$

The Mie phase function is given in equation 2.10 where $S_{1}(\theta)$ (equation 2.11) and $S_{2}(\theta)$ (equation 2.12) are the scattering amplitude functions which involve infinite sums of both the scattering coefficients and angle-dependent functions.

$$
\begin{gathered}
p_{\mathrm{M}}=\frac{1}{\sigma_{\mathrm{sca}}} \frac{\left|S_{1}(\theta)\right|^{2}+\left|S_{2}(\theta)\right|^{2}}{2 k^{2}} \\
S_{1}(\theta)=\sum_{m=1}^{\infty} \frac{2 m+1}{m(m+1)}\left(a_{m} \pi_{m}+b_{m} \tau_{m}\right)
\end{gathered}
$$




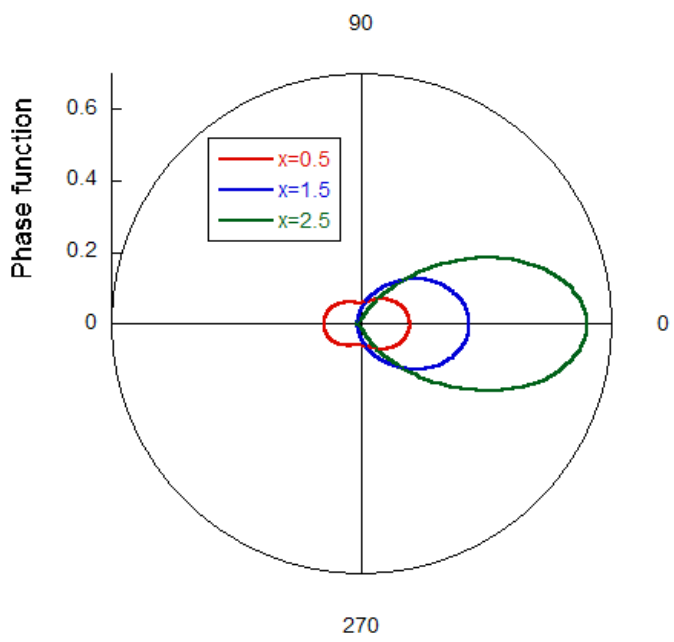

(a)

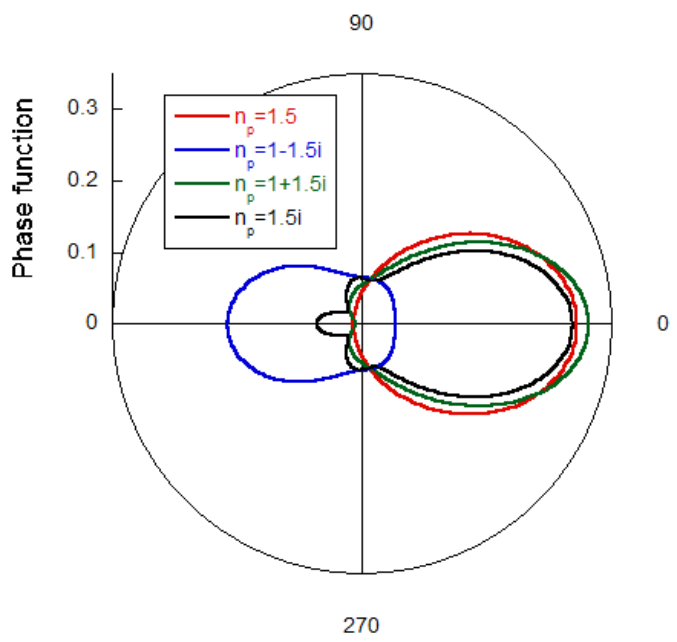

(b)

Figure 2.3: Mie phase function for (a) various size parameters with $n_{p}=1.5$ and (b) various particle refractive indices with $x=1.5$. Both have a fixed medium refractive index of $n_{m}=1$.

$$
S_{2}(\theta)=\sum_{m=1}^{\infty} \frac{2 m+1}{m(m+1)}\left(a_{m} \tau_{m}+b_{m} \pi_{m}\right)
$$

For particles which are small compared to the wavelength of light, Mie theory has an approximate analytical solution. An assumption that $|n| x \ll 1$ is made which requires not only the radius to be much smaller than the wavelength but also for the field to penetrate readily into the particle. Taking terms of order up to $x^{4}$ the scattering and absorption cross-sections are given by equation 2.13 and 2.14 respectively.

$$
\begin{gathered}
\sigma_{\mathrm{sca}}=\frac{128 \pi^{5}}{3} \frac{a^{6}}{\lambda^{4}} n_{\mathrm{m}}^{2}\left|\frac{n^{2}-1}{n^{2}+2}\right|^{2} \\
\sigma_{\mathrm{abs}}=\frac{8 \pi a}{\lambda} n_{\mathrm{m}} \operatorname{Im}\left\{\frac{n^{2}-1}{n^{2}+2}\right\}
\end{gathered}
$$

The scattering cross-section given here is exactly the same as equation 2.3 for real $n$, meaning for small dielectric particles Mie theory reduces to Rayleigh scattering.

For large particles the equations have to be solved numerically with the use of a computer. Since infinite sums cannot be calculated the series has to be truncated at a sufficiently large number of terms in order to achieve an accu- 
rate result. Bohren and Huffman [12] suggest for numeric experiments that the nearest integer to $x+4 x^{1 / 3}+2$, where $x$ is the size parameter can be used as the number of terms required. Computational algorithms for Mie theory were written using MATLAB and were based on previous work by Christian Mätzler [13]. Results of the Mie phase function for various size parameters and refractive indices are shown in figure 2.3. These graphs show for a large size parameter the scattering occurs predominately in the forwards direction. The refractive index also has a significant impact on the shape of the curve; particles with a negative imaginary refractive index scatter in the reverse direction.

\subsubsection{Henyey-Greenstein approximation}

Glass ceramic materials are formed as a glass and then heat treated (annealed) to induce crystallisation. It is these crystallites which cause the storage phosphor effect and from which scattering occurs. Depending on the type of annealing, crystallites of sizes from $10-100 \mathrm{~nm}$ can be produced (see section 2.1), and so in any modelling of a glass ceramic imaging plate, a scattering theory which works with a range of particle sizes needs to be used. The ideal scattering theory would be Mie theory. However, Mie theory is very time consuming for a large number of scattering events so here the Henyey-Greenstein phase function is used as a computationally simpler approximation.

The Henyey-Greenstein phase function [14] was developed to model the scattering of diffuse radiation in the Galaxy by dust. The function $\Phi$ (equation 2.15) defines the direction of scattering by an angle $\theta$ which is the deviation from the forwards direction and a factor $g$ known as the anisotropy coefficient.

$$
\Phi(\theta)=\frac{1-g^{2}}{4 \pi\left(1+g^{2}-2 g \cos \theta\right)^{\frac{3}{2}}}
$$

The anisotropy coefficient describes how strongly the scattering deviates from spherical scattering. For $g=0$ the scattering is isotropic and for $g=1$ all of 


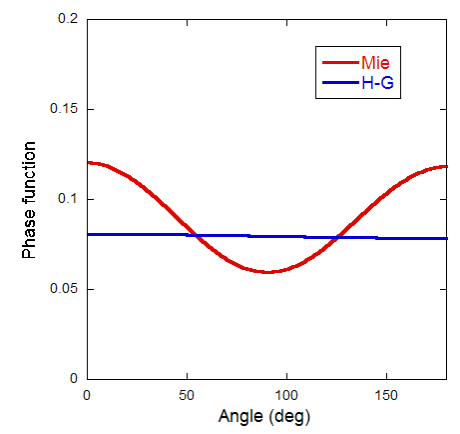

(a)

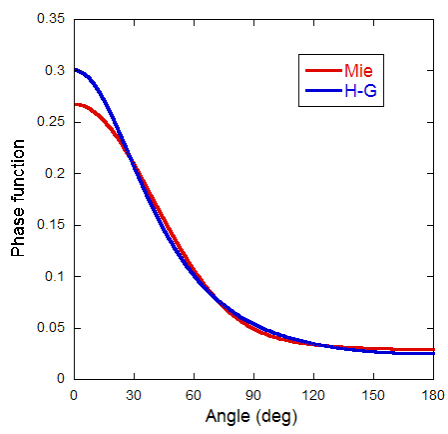

(b)

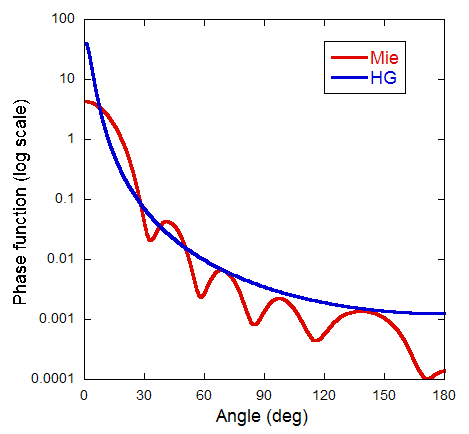

(c)

Figure 2.4: Plots of Henyey-Greenstein phase function versus Mie phase function for particles with radius (a) $a=10 \mathrm{~nm}$, (b) $a=100 \mathrm{~nm}$ and (c) $a=500$ nm.

the light is scattered in the forwards direction. A negative $g$ corresponds to the scattering tending towards the backwards direction. The anisotropy coefficient is defined as the average cosine of the phase function, $p(\theta)$.

$$
g=2 \pi \int_{0}^{\pi} p(\theta) \cos \theta \sin \theta d \theta
$$

Figure 2.4 shows how the Henyey-Greenstein function compares with Mie theory for various particle sizes. In each case the anisotropy coefficient was determined by substituting the Mie phase function for the particular particle size into equation 2.16 and numerically integrating over $\theta$. The Henyey-Greenstein function is a good approximation to Mie theory for particles with radius $\sim 100$ $\mathrm{nm}$ (size parameter of approximately one).

\subsection{Reflection}

When a light wave passes between media of differing refractive indices reflection and refraction of the light may occur as shown in figure 2.5. The angles at which the reflected and refracted waves travel with respect to the normal of the interface are given by the law of reflection (2.17) and Snell's law (2.18).

$$
\theta_{\mathrm{i}}=\theta_{\mathrm{r}}
$$




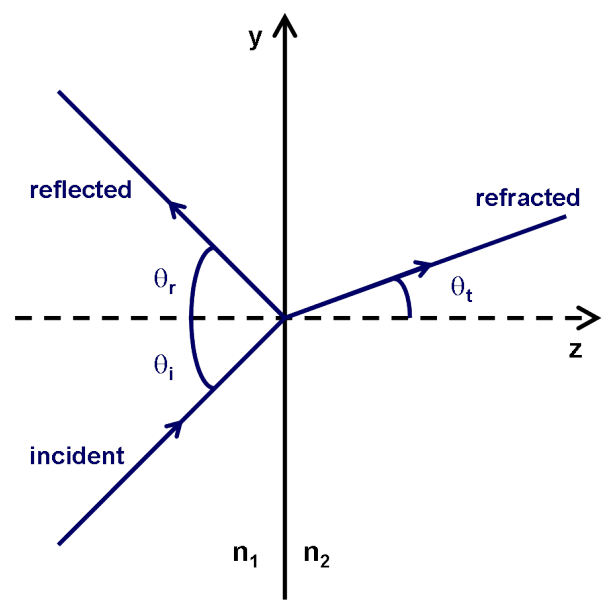

Figure 2.5: Diagram of reflected and refracted waves at a boundary between media of different refractive indices.

$$
n_{1} \sin \theta_{\mathrm{i}}=n_{2} \sin \theta_{\mathrm{t}}
$$

In imaging plates this occurs when the laser or PSL light is incident at the surfaces between plate material and their surroundings. To implement reflection in computations the Fresnel equations are used. Treatment of the Fresnel equations can be found in any elementary electromagnetism text book, for example Griffiths [15].

The Fresnel equations were developed by Augustin-Jean Fresnel to explain the behavior of light at the boundary between media of different refractive indices. These equations are given in terms of a reflection and transmission coefficient which give the fraction of the intensity of incident light which is either reflected or transmitted. The different polarisations for the incident light wave lead to different Fresnel equations. The Fresnel equations for perpendicular and parallel polarisation are given in equations 2.19 to 2.22.

$$
\begin{gathered}
R_{\perp}=\left(\frac{n_{1} \cos \theta_{\mathrm{i}}-n_{2} \cos \theta_{\mathrm{t}}}{n_{1} \cos \theta_{\mathrm{i}}+n_{2} \cos \theta_{\mathrm{t}}}\right)^{2} \\
T_{\perp}=\frac{n_{2} \cos \theta_{\mathrm{t}}}{n_{1} \cos \theta_{\mathrm{i}}}\left(\frac{2 n_{1} \cos \theta_{\mathrm{i}}}{n_{1} \cos \theta_{\mathrm{i}}+n_{2} \cos \theta_{\mathrm{t}}}\right)^{2} \\
R_{\|}=\left(\frac{n_{2} \cos \theta_{\mathrm{i}}-n_{1} \cos \theta_{\mathrm{t}}}{n_{2} \cos \theta_{\mathrm{i}}+n_{1} \cos \theta_{\mathrm{t}}}\right)^{2}
\end{gathered}
$$




$$
T_{\|}=\frac{n_{2} \cos \theta_{\mathrm{t}}}{n_{1} \cos \theta_{\mathrm{i}}}\left(\frac{2 n_{1} \cos \theta_{\mathrm{i}}}{n_{1} \cos \theta_{\mathrm{i}}+n_{2} \cos \theta_{\mathrm{t}}}\right)^{2}
$$

Due to energy conservation the reflection coefficient and transmission coefficient must always add to one.

If the incident light is unpolarised then the reflection and transmission coefficients are given by the average of perpendicular and parallel polarisation, as shown below.

$$
\begin{gathered}
R=\frac{1}{2}\left[\left(\frac{n_{1} \cos \theta_{\mathrm{i}}-n_{2} \cos \theta_{\mathrm{t}}}{n_{1} \cos \theta_{\mathrm{i}}+n_{2} \cos \theta_{\mathrm{t}}}\right)^{2}+\left(\frac{n_{2} \cos \theta_{\mathrm{i}}-n_{1} \cos \theta_{\mathrm{t}}}{n_{2} \cos \theta_{\mathrm{i}}+n_{1} \cos \theta_{\mathrm{t}}}\right)^{2}\right] \\
T=\frac{1}{2} \frac{n_{2} \cos \theta_{\mathrm{t}}}{n_{1} \cos \theta_{\mathrm{i}}}\left[\left(\frac{2 n_{1} \cos \theta_{\mathrm{i}}}{n_{1} \cos \theta_{\mathrm{i}}+n_{2} \cos \theta_{\mathrm{t}}}\right)^{2}+\left(\frac{2 n_{1} \cos \theta_{\mathrm{i}}}{n_{1} \cos \theta_{\mathrm{i}}+n_{2} \cos \theta_{\mathrm{t}}}\right)^{2}\right]
\end{gathered}
$$

When the light wave is incident normal to the boundary the Fresnel equations reduce to equations 2.25 and 2.26 .

$$
\begin{gathered}
R=R_{\perp}=R_{\|}=\left(\frac{n_{1}-n_{2}}{n_{1}+n_{2}}\right)^{2} \\
T=T_{\perp}=T_{\|}=\frac{4 n_{1} n_{2}}{\left(n_{1}+n_{2}\right)^{2}}
\end{gathered}
$$

\subsection{Modulation transfer function}

Spatial resolution is defined as the ability to distinguish the shape or extent of features within an image and depends on the properties of the system which creates the image.

A periodic image can be expressed as a sum of a series of harmonic sinusoids in image brightness with the use of Fourier series theory. For non periodic images the Fourier transform $F(\nu)$ ( $\nu$ is the spatial freqency) of the image brightness $f(x)$ (equation 2.27) contains the equivalent information about the image 
(a) lcad grid

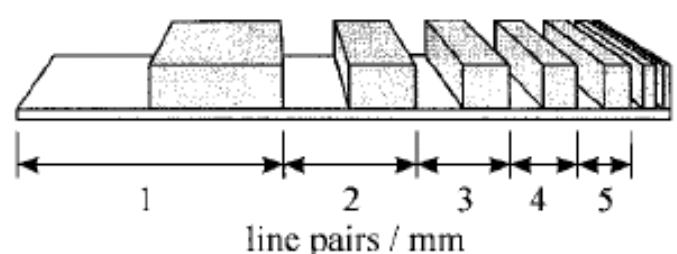

(b) ideal image

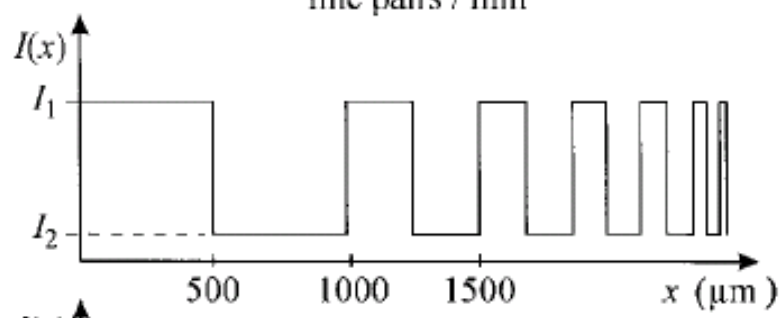

(c) real image

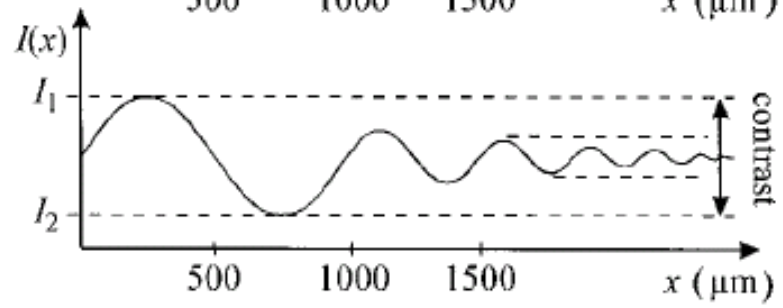

Figure 2.6: Determination of the MTF by imaging a lead grid. [16]

brightness in the frequency domain.

$$
F(\nu)=\frac{1}{\sqrt{2 \pi}} \int_{-\infty}^{\infty} f(x) e^{-i \nu x} d x
$$

An important measure of the spatial resolution of an imaging plate is the modulation transfer function (MTF). The MTF is defined as the ratio of the contrast in the read-out image to the contrast of the original image, and is a function of the spatial frequency. The MTF is mathematically equivalent to the normalised Fourier transform of the line spread function (LSF), which is the read-out image as a function of distance from an ideal line image of infinitesimal width. In practical applications, the MTF can be found in two different ways.

The first (figure 2.6) is to obtain an image of a periodic test object, which consists of a series of vertical lines at small known spacings, on the imaging plate. An example of a test object is a lead grid where the spatial frequency (line pairs per $\mathrm{mm}$ ) changes stepwise between each set. The plate is then scanned in a direction perpendicular to the lines with a laser, the response being a signal which is modulated by the lines. This response of the imaging plate generates 

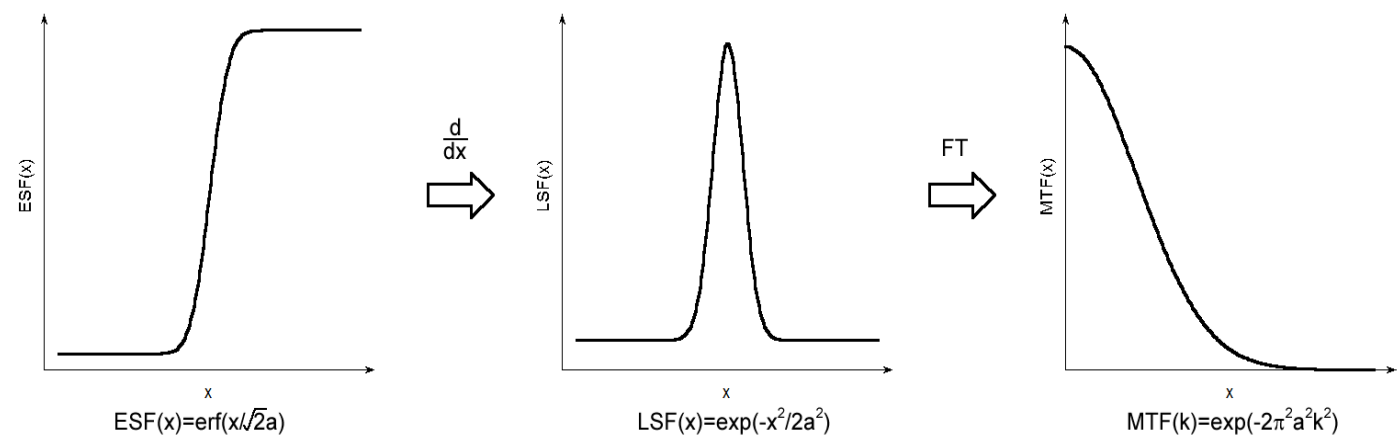

Figure 2.7: Determination of the MTF by imaging a sharp edge.

the contrast transfer function (CTF) which can be transformed into the MTF by approximating the square wave pattern as a series of sinusoidal functions.

The second way is to scan across an image of a sharp edge, the response is known as the edge spread function (ESF). Subsequent differentiation and Fourier transformation of the ESF determines the MTF as shown schematically in figure 2.7 .

\subsection{Literature review}

Several authors have investigated the influence of scattering on the spatial resolution of storage phosphor imaging plates. In particular two papers, Thoms [17] and Fasbender et al [18], have modelled the scattering of light using computational methods.

Thoms [17] treats the scattering of light as a diffusion-like process and develops theoretical equations which can be solved numerically using standard computational methods. The model finds that by increasing the absorption, decreasing the scattering length or decreasing the plate thickness the resolution will be increased at a cost of a decrease in the sensitivity. To achieve a specific resolution it is suggested that the thickness of the plate should be as large as possible, the absorbance of the medium as small as possible and then the resolution is fitted by adjusting the scattering length of the medium. The model has several limitations. The scattering from particles is assumed to be 
isotropic implying small particle sizes which does not include $\mathrm{BaBrF}: \mathrm{Eu}^{2+}$ with particles around $8 \mu \mathrm{m}$ in size or glass ceramics with crystallite sizes of $100 \mathrm{~nm}$ or larger. The scattering length is assumed to be much smaller than the plate width, which excludes transparent materials such as glass ceramics. Bleaching of the image during read-out is not included, nor is reflection from any surface.

Fasbender et al [18] model the scattering of light using a Monte Carlo algorithm where the incident laser light is split up into packets of photons. The packets are followed as they travel through the plate where they experience numerous random events until they leave from the plate surfaces or are absorbed by the medium. Random events are determined with the use of probability distributions and randomly generated numbers. The scattering distribution allows for anisotropic scattering with the use of the Henyey-Greenstein function (see section 2.2.3). Once the laser packets have been followed through the plate the intensity distributions are found by mapping the photon paths onto a two dimensional matrix where the rows and columns represent the spatial positions and each entry is the photon density within that range of values. The PSL photons experience similar random scattering events to the laser photons. Of interest is the escape probability (from top or bottom surface) of PSL photons generated on a light-emitting $x-y$ plane at a given depth in the plate. After generation, the initial direction of the PSL photons is assumed to be isotropic. Based on the probability of escape and intensity distribution of the laser, the LSF and MTF can be determined. In addition, bleaching of the line is considered with the number of storage centres after scanning a certain distance decreasing with laser intensity. A series of storage phosphor plates of varying thicknesses where then created by Fasbender et al to determine their resolution and sensitivity. The MTF was determined experimentally from the square wave response function of a periodic bar pattern and the sensitivity was measured using an Ulbricht sphere to integrate the PSL photons. The 


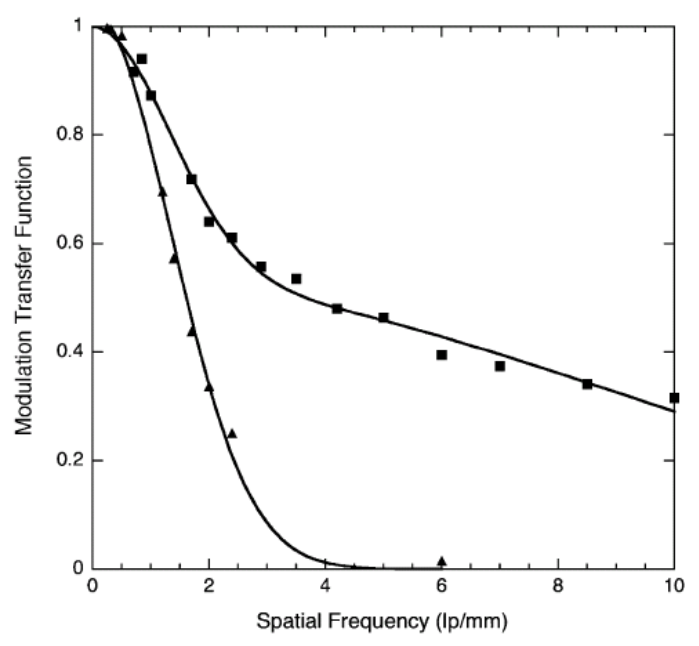

Figure 2.8: MTF's for a glass ceramic (squares) and Agfa MD30 (triangles) imaging plate [19].

simulation and experimental results show that increasing the plate thickness decreases the resolution and increases the sensitivity. Simulated scattering profiles show that increasing the optical absorption coefficient decreases the scattering area and increases the resolution. This model does not include reflection from surfaces and only investigates plate widths which are much greater than the scattering length.

Several authors have investigated the spatial resolution of glass ceramics experimentally and their ability to be used as imaging plates.

Edgar et al [19] determined the modulation transfer function of a flurochlorozirconate glass doped with $1 \mathrm{~mol} \% \mathrm{Eu}^{2+}$ and a commercial Agfa imaging plate by point-by-point scanning across an image of a bar pattern (figure 2.8). The spatial resolution for a translucent glass ceramic was found to be a considerable improvement over that for commercial plates. The MTF for the glass ceramic was found to have a bimodal nature, which was accredited to the finite laser beam width for the high frequency regime and the surrounding scatter halo in the low frequency regime.

Chen et al [20] evaluate the MTF using a bar pattern phantom and an extended laser beam which reads out all of the irradiated image. The glass ceramics used are based on the ZBLAN20 composition with varying Eu ${ }^{2+}$ concentra- 
tions and annealed using either a one or two step method. A two step annealing treatment produces a larger number of smaller crystallites. The resolution of a single annealed $2 \% \mathrm{Eu}^{2+}$ doped sample was found to be approximately $30 \mathrm{lp} / \mathrm{mm}$. A double annealed $1 \% \mathrm{Eu}^{2+}$ doped sample has a resolution of approximately $90 \mathrm{lp} / \mathrm{mm}$ at a MTF of 0.2 .

Schweizer and Johnson [21] investigated the spatial resolution using the same glass ceramics and methods as in Chen et al [20]. Measurements on a double annealed $2 \% \mathrm{Eu}^{2+}$ doped glass show a resolution of $110 \mathrm{lp} / \mathrm{mm}$ at an MTF of 0.2 .

It should be noted that the read-out of Chen et al and Schweizer and Johnson was not by a scanned laser beam but by total illumination of the plate and image recording using a CCD based camera. In this case the resolution is limited not by the scattering of the stimulating laser light but by scattering of the PSL light. 



\section{Chapter 3}

\section{Scattering Simulations}

\subsection{Monte Carlo methods}

A Monte Carlo simulation is a method for iteratively evaluating a model with the use of random numbers and probability statistics. A simple example of a Monte Carlo method is to calculate the area of a unit circle using a "hit and miss" method which leads to an estimation of $\pi$. Mathematically the area is given by $A=4 \int_{0}^{\sqrt{1-x^{2}}} \int_{0}^{1} d x d y=\pi$.

The Monte Carlo method is applied by imagining throwing darts at one quadrant of the square in figure 3.1. The number of darts landing in the shaded part, tested by comparing the radius of impact with one, divided by the total number of darts is equal to the ratio of the two areas.

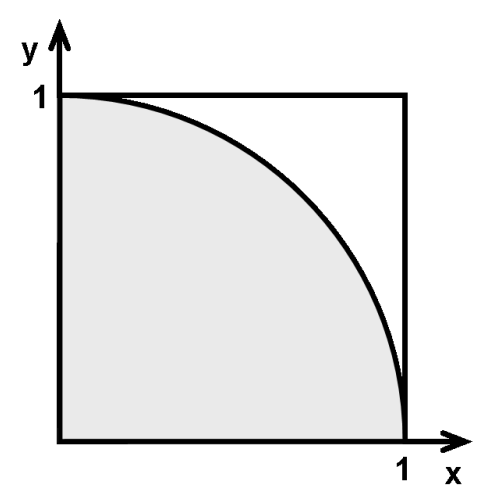

Figure 3.1: Quadrant of circle in box for Monte Carlo determination of $\pi$. 


$$
\frac{\# \text { darts hitting shaded part }}{\# \text { darts hitting inside square }}=\frac{\text { area of shaded part }}{\text { area of square }}
$$

An estimation of $\pi$ is then given by equation 3.2. A very large number of throws are required to get an accurate value of $\pi$.

$$
\pi=4 \frac{\# \text { darts hitting shaded part }}{\# \text { darts hitting inside square }}
$$

Monte Carlo simulations were set up to describe the light scattering in storage phosphor imaging plates and closely follows the model developed by Fasbender et al [18]. To apply the method, the laser light which is incident on the plate during read-out of the image is split up into a large number of packets which are followed as they travel through the plate. Monte Carlo techniques are used to determine simulation events such as the position photon packets land on the plate, the distance packets travel before scattering and the angle at which packets are scattered.

\subsection{Random number generation}

Monte Carlo methods rely on random numbers to compute their results. In this work, uniform random deviates between 0.0 and 1.0 are generated by two random number generators. Two generators are used to produce the most randomly distributed number possible. The first random number generator is a Park-Miller generator with a period of about $3.1 \times 10^{18}$ [22]. This generator requires a negative integer seed to initialise the number generation and for a given seed the list of generated numbers is exactly the same. Therefore, a second random number generator [23] is used to generate a random seed which will change the list of generated numbers. The second generator uses the computer's clock to generate a random sequence of numbers but suffers from a periodic pattern of numbers, so cannot be used on its own. To generate the seed for the first generator an average of two random numbers from the sec- 
ond generator is multiplied by -100 to give a seed value between -100 and 0 .

\subsection{Overview of the model}

As mentioned previously, the incident read-out laser light is split up into a number of packets, each containing a set number of photons. These packets are followed step-wise as they advance through the plate, where they undergo numerous events such as scattering, absorption by the material and/or storage centres and reflection off plate surfaces. Intensity distribution profiles of the scattered laser light are constructed by splitting the plate into a number of volume units (voxels) and determining the density of photons in that volume. Due to spherical symmetry of the laser beam, these intensity distributions are represented in terms of $r$ and $z$ coordinates.

Once stimulated the PSL photons will also undergo similar numerous events to the laser photons. To simulate this, packets of photons are generated at a number of depths in the plate, and these are followed step-wise through the plate. The number of photons leaving through a surface of the plate is recorded and this generates the probability of escape of PSL photons from a plate surface for a given depth.

Both the laser intensity distributions and PSL probability of escapes are then used to determine the LSF. The laser beam is scanned across a thin line of irradiated centres and the intensity of the stimulated PSL is collected as a function of distance from the line. The MTF is then determined by Fourier transforming and normalising the LSF, from which the resolution of the plate can be determined.

All code was written in the Fortran 90 language and was compiled using the free gfortran compiler for windows available on the internet [24]. Typical computation times ranged from an average of 8 hours for intensity distribution 
and LSF calculations to an average of 48 hours for probability of escape calculations. To compensate for large computation times, often several computers (up to a maximum of 20) were used with each computer running a different simulation.

\subsection{Photon packet description of the laser beam}

The number of photons in a packet, $N_{\text {pht }}$ (equation 3.3) is a function of the power of the read-out laser, $P_{\text {las, }}$ energy of the laser photons, $E_{\text {pht }}$, velocity of the read-out scanner, $v_{\text {scan }}$, the sampling interval, $\Delta x$, and the number of packets the total beam is split into, $N_{\mathrm{pkt}}$.

$$
N_{\mathrm{pht}}=\frac{P_{\mathrm{las}} \Delta x}{E_{\mathrm{pht}} v_{\mathrm{scan}} N_{\mathrm{pkt}}}
$$

The intensity of the incident laser beam over its cross-section is assumed to be Gaussian distributed with variance $w$. Since the laser beam is perpendicularly incident on the plate, cylindrical geometry can be applied leading to an intensity distribution as given in equation 3.4.

$$
I(r) \propto \frac{1}{\sqrt{2 \pi} w} \exp \left(\frac{-r^{2}}{2 w^{2}}\right)
$$

To find where the photon packet lands on the plate the probability density function (equation 3.5) is found by the normalisation condition for a beam of unit power, $\int_{0}^{\infty} I(r) 2 \pi r d r=1$ and then multiplying through by $2 \pi r$.

$$
p(r)=\frac{r}{w^{2}} \exp \left(\frac{-r^{2}}{2 w^{2}}\right)
$$

A Monte Carlo technique can be applied to determine the positions the photon packets land on the plate $(r)$. To do this a set of values of $r$ need to be generated which will follow the probability density distribution $p(r)$. Figure 3.2(a) shows 


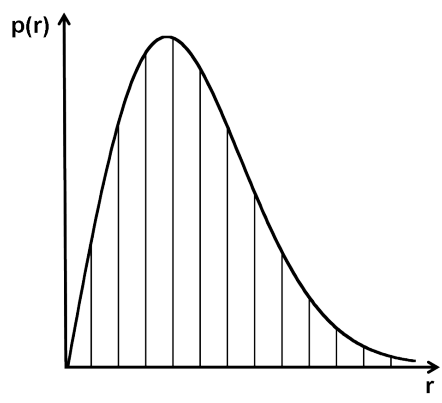

(a)

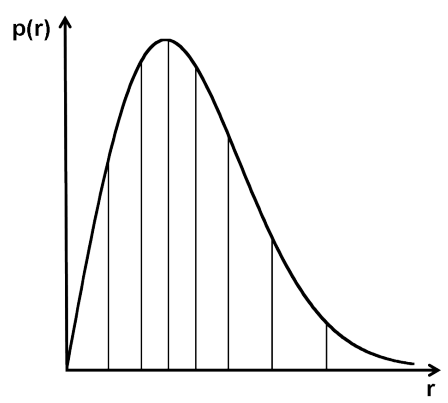

(b)

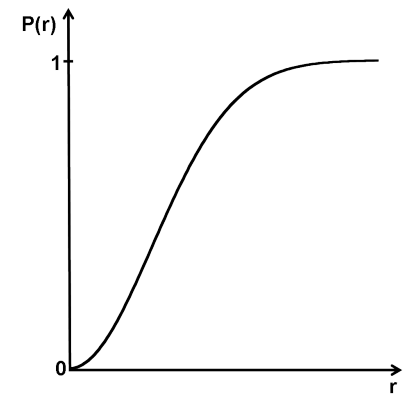

(c)

Figure 3.2: Illustration of probability density function with partitioning of $r$ on basis of (a) equal $\Delta r$ spacing and (b) equal area spacing and (c) illustration of probability distribution function.

the probability density function with the range of $r$ values divided into increments of $\Delta r$. Imagine throwing darts at this figure so that the number of darts per unit area is uniform; the number of darts in each box is then proportional to the area $p(r) \Delta r$ of the box. If instead the boxes are organised on the basis of equal areas (figure 3.2(b)), the number of darts in each box will be the same and those values of $r$ are equally likely to occur. Generating random numbers between 1 and the number of boxes, the linear density of corresponding $r$ values is a good approximation to $p(r)$. This process is equivalent to taking the integral of $p(r), P(x)=\int_{0}^{r} p(z) d z$ (figure 3.2(c)) which extends from 0 to 1 and dividing it up into equal segments. By choosing a value of $P(r)$ between 0 and 1 (just like random number generators) a particular value of $r$ can be found. A large number $r$ values can be generated by this process, all of which will follow the distribution of $p(r)$.

This is an example where a given normalised probability density function $p(z)$ which satisfies $\int_{a}^{b} p(z) d z=1$ has been simulated by taking the probability distribution function $P(x)=\int_{a}^{x} p(z) d z$ to be uniformly distributed over this interval $(a, b)$ and generating sets of $z$ which satisfy $p(z)$ from it using sets of $P$ selected by a random number generator.

Using this technique equation 3.5 is changed into the probability distribution 


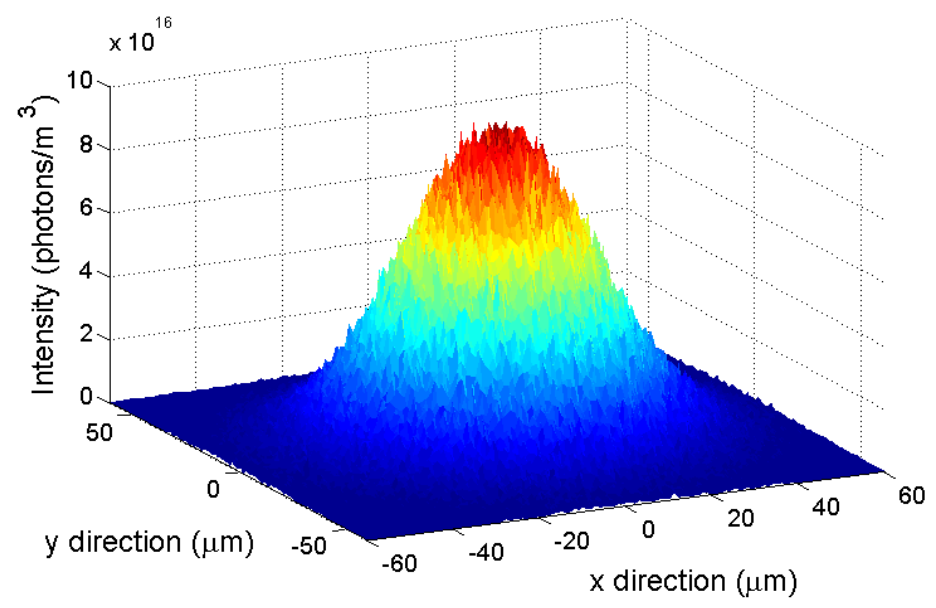

Figure 3.3: Simulated laser beam incident on plate for a beam variance of $w=$ $20 \mu \mathrm{m}$.

function equation (3.6).

$$
P(r)=1-\exp \left(\frac{-r^{2}}{2 s^{2}}\right)
$$

The simulation then generates two random numbers, $R_{r}$ and $R_{\alpha}$ to find the radial (equation 3.7) and angular (equation 3.8) landing positions of the photon packet.

$$
\begin{gathered}
r=w \sqrt{-2 \ln \left(1-R_{\mathrm{r}}\right)} \\
\alpha=2 \pi R_{\alpha}
\end{gathered}
$$

The radial position is found by substituting $R_{\mathrm{r}}$ for $P(r)$ and rearranging for $r$. The angular position is equally distributed in the range of 0 to $2 \pi$.

A simulation of the intensity distribution, based on the spatial distribution of photon packets calculated by these methods is shown in figure 3.3.

\subsection{Simulation of the scattering process}

Scattering of the laser and PSL light is caused by variations in the refractive index of the imaging plate; in practice by the granular nature of a ceramic or the crystalline content of a glass ceramic. To simulate this the photon packet 


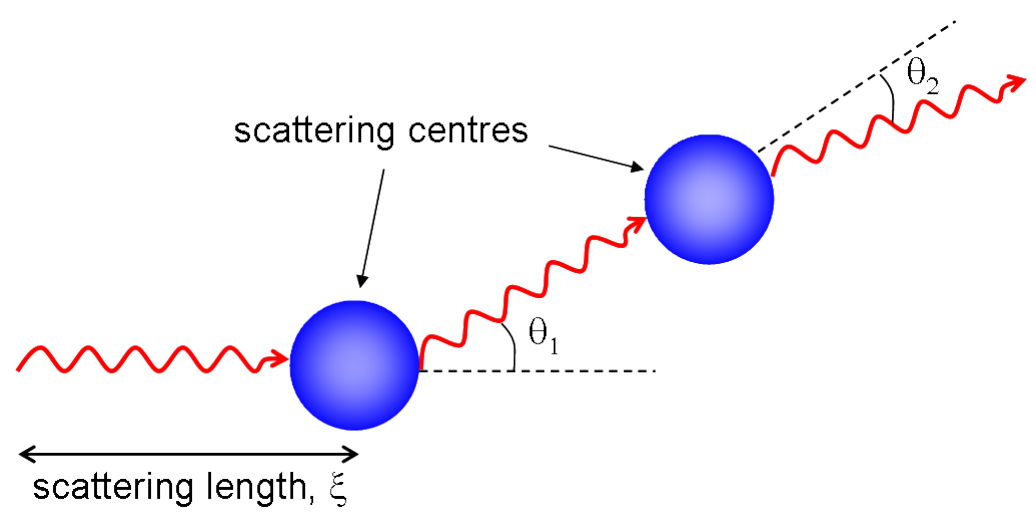

Figure 3.4: Scattering of photon packets within the plate.

travels a randomly determined distance $\xi$, is scattered, and then travels in a different direction as illustrated in figure 3.4. In order to determine this distance, the overall form of the scattering is assumed to follow the Beer-Lambert law, equation 3.9 where $N_{\text {scat }}$ is the number of scattered photons from an initial number $N_{\text {pht }}$ after travelling a distance $\xi$.

$$
N_{\text {scat }}=N_{\text {pht }}\left(1-\exp \left(-\frac{\xi}{\mu}\right)\right)
$$

The probability distribution function for a packet to be scattered in the range 0 to $\xi$ comes directly from equation 3.9.

$$
P(\xi)=1-\exp \left(-\frac{\lambda}{\xi}\right)
$$

The length $\xi$ can then be determined by generating a random number $R_{\xi}$ to replace $P(\xi)$ and then rearranging for $\xi$.

$$
\xi=-\mu \ln \left(1-R_{\xi}\right)
$$

After travelling distance $\xi$, the packet is scattered by a scattering centre and deviates by angles $\theta$ and $\phi$ from the previous direction of travel. The azimuthal angle, $\phi$, is evenly distributed between 0 and $2 \pi$ because of rotational symmetry, and the distribution of $\theta$ between 0 and $\pi$ depends on the anisotropy of the 
scattering particles.

The Henyey-Greenstein phase function (equation 3.12) is a good approximation for anisotropic scattering from particles as explained in section 2.2.3.

$$
\Phi(\theta)=\frac{1-g^{2}}{4 \pi\left(1+g^{2}-2 g \cos \theta\right)^{\frac{3}{2}}}
$$

Values for the anisotropy coefficient $g$ are chosen to correspond to particles with radii ranging from $10 \mathrm{~nm}$ to $1 \mu \mathrm{m}$. The probability distribution for $\theta$ can be found by integrating $\Phi(\theta)$ over a sphere.

$$
P(\theta)=\int_{0}^{2 \pi} \int_{\theta}^{\pi} \Phi(\theta) \sin \theta d \theta d \phi=\frac{1-g^{2}}{2 g}\left[\frac{1}{\sqrt{1+g^{2}-2 g \cos \theta}}-\frac{1}{g+1}\right]
$$

Introducing random numbers $R_{\theta}$ for $P(\theta)$ and $R_{\phi}$ gives equations 3.14 and 3.15 for $\theta$ and $\phi$ respectively.

$$
\begin{gathered}
\theta=\cos ^{-1}\left(\frac{1+g^{2}-\left[\left(1-g^{2}\right) /\left(1-g+2 g R_{\theta}\right)\right]^{2}}{2 g}\right) \\
\phi=2 \pi R_{\phi}
\end{gathered}
$$

The new positions of the packet after being scattered are found in terms of the previous angles $\theta_{1}$ and $\phi_{1}$, and the change in direction given by angles $\theta$ and $\phi$. There are three different sets of coordinate systems that are used - the base $(x, y, z)$ system, the previous direction $\left(x_{1}, y_{1}, z_{1}\right)$ system and the new $\left(x_{2}, y_{2}, z_{2}\right)$ system. The new packet position needs to be specified in terms of the $(x, y, z)$ coordinate system.

Firstly, the change in the $\left(x_{1}, y_{1}, z_{1}\right)$ system due to scattering angles $\theta$ and $\phi$ is determined. A rotation about $y_{1}$ by $\theta$ and then rotating anticlockwise about $z_{1}$ by $\phi$ gives equation 3.16, which is the new position vector in terms of the $\left(x_{1}, y_{1}, z_{1}\right)$ coordinate system.

$$
(-\sin \theta \cos \phi,-\sin \theta \sin \phi, \cos \theta)
$$




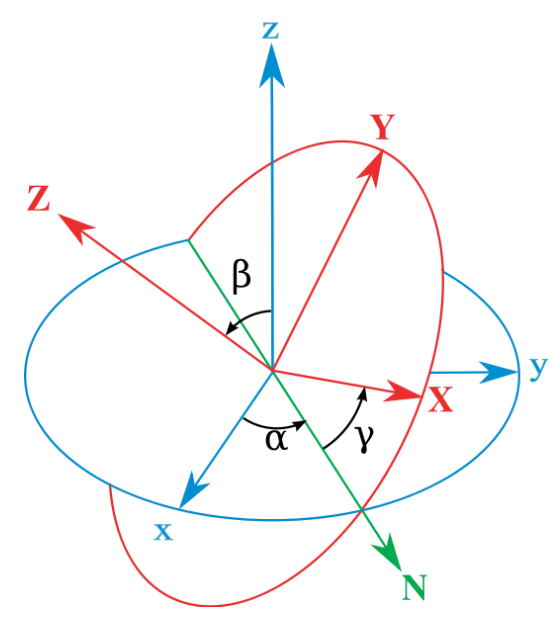

Figure 3.5: Euler angle geometry - the xyz (fixed) system is shown in blue, the $\mathrm{XYZ}$ (rotated) system is shown in red. The line of nodes, labeled $\mathrm{N}$, is shown in green. [25]

To change this into the $(x, y, z)$ system an inverse Euler transformation is used, with transformation matrix given by equation 3.17. The Euler angles are illustrated in figure 3.5 where $\alpha=90^{\circ}, \beta=\theta_{1}$ and $\gamma=\phi_{1}+90$ in relation to this system.

$$
A^{-1}=\left(\begin{array}{ccc}
-\cos \theta_{1} \cos \phi_{1} & \sin \theta_{1} & \sin \theta_{1} \cos \phi_{1} \\
-\cos \theta_{1} \sin \phi_{1} & -\cos \phi_{1} & \sin \theta_{1} \sin \phi_{1} \\
\sin \theta_{1} & 0 & \cos \theta_{1}
\end{array}\right)
$$

Multiplying the Euler matrix by the new position vector gives the positions after being scattered in the $(x, y, z)$ system (equation 3.18).

$$
\begin{gathered}
x_{2}=\sin \theta_{2} \cos \phi_{2}=\left(u \cos \theta_{1}+w \sin \theta_{1}\right) \cos \phi_{1}-v \sin \phi_{1} \\
y_{2}=\sin \theta_{2} \sin \phi_{2}=\left(u \cos \theta_{1}+w \sin \theta_{1}\right) \sin \phi_{1}+v \cos \phi_{1} \\
z_{2}=\cos \theta_{2}=w \cos \theta_{1}-u \sin \theta_{1}
\end{gathered}
$$

where

$$
\begin{gathered}
u=\sin \theta \cos \phi \\
v=\sin \theta \sin \phi \\
w=\cos \theta
\end{gathered}
$$




\subsection{Absorption of photons}

The packet is followed as it advances through the plate in small steps of $\Delta \rho$ $\mu \mathrm{m}$ in the direction of travel. In each step the number of photons in the packet decreases due to absorption by the material for example by storage centres and is given by equation 3.20 where $\gamma$ is the optical absorption coefficient.

$$
N_{i}=N_{i-1} \exp (-\gamma \Delta \rho)
$$

\subsection{Reflection from surfaces}

After travelling through the plate the packet reaches either the front or rear surface, where reflection at that surface occurs. Reflection is included in the simulation with the use of Fresnel's reflection equation for unpolarised light (equation 3.21), where $n_{1}$ is the refractive index of the medium, $n_{2}$ the refractive index of the surroundings, $\theta_{\mathrm{i}}$ the angle of incidence and $\theta_{\mathrm{t}}$ the angle of transmission or refraction. $\theta_{\mathrm{t}}$ is given by Snell's law, $n_{1} \sin \theta_{\mathrm{i}}=n_{2} \sin \theta_{\mathrm{t}}$.

$$
R=\frac{1}{2}\left[\left(\frac{n_{1} \cos \theta_{\mathrm{i}}-n_{2} \cos \theta_{\mathrm{t}}}{n_{1} \cos \theta_{\mathrm{i}}+n_{2} \cos \theta_{\mathrm{t}}}\right)^{2}+\left(\frac{n_{2} \cos \theta_{\mathrm{i}}-n_{1} \cos \theta_{\mathrm{t}}}{n_{2} \cos \theta_{\mathrm{i}}+n_{1} \cos \theta_{\mathrm{t}}}\right)^{2}\right]
$$

When the photon packet is incident on a surface the number of photons which are reflected is calculated, and then the packet continues through the plate with this number of photons. This continues until only a small number of photons remain.

\subsection{Intensity distribution profiles}

Intensity distribution profiles are generated to show the nature of the scattering of laser photons through the plate. After each packet has travelled through the plate, the section of the imaging plate required is split into a number of vol- 


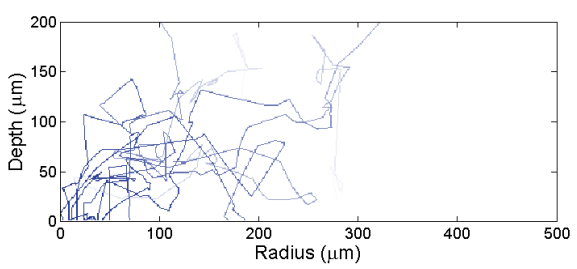

(a) 10 packets

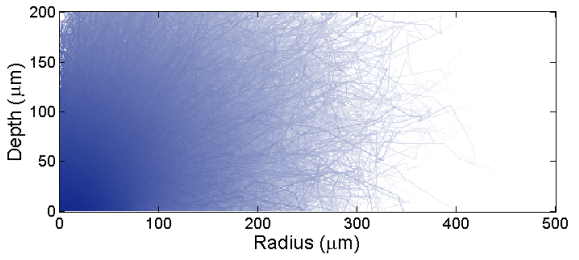

(c) 10000 packets

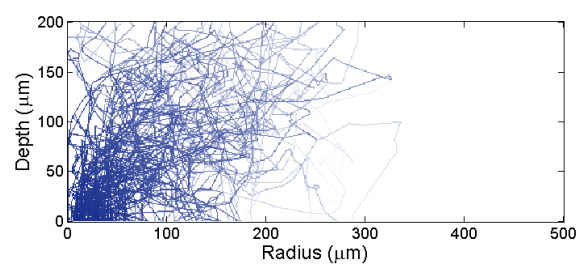

(b) 100 packets

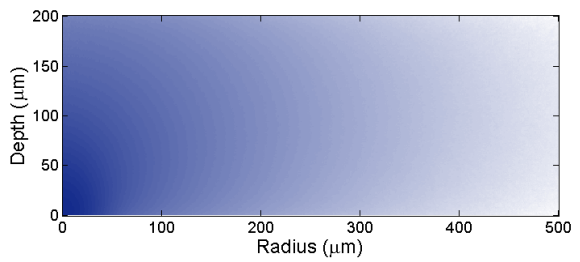

(d) 1000000 packets

Figure 3.6: Scattering profiles of the incident read-out laser with $w=20 \mu \mathrm{m}$ after following a set number of packets (as shown) through the plate.

ume units (voxels) and the trace created by the packet is mapped onto the grid of voxels. The corresponding laser photon density due to the single trace is added to each voxel and this continues until all packets have travelled through the plate. Due to the cylindrical symmetry of the laser beam, the volume units can be converted to area units using $r$ and $z$ coordinates. The intensity profiles of the scattered laser light within the plate are then formed by creating a matrix $I(r, z)$, where each entry is the photon density within the range of corresponding points $\left(r_{i}-r_{i-1}, z_{i}-z_{i-1}\right)$. Figure 3.8 shows the intensity distribution after following a set number of packets through the plate. By increasing the number of packets more accurate results are achieved.

\subsection{Probability of escape}

When the laser light is incident on a storage centre, photostimulated luminescence (PSL) photons can be emitted. These PSL photons are emitted at a rate proportional to the density of laser photons in the plate, as given by equation 3.22 where $n_{\mathrm{em}}$ is the emitted-photon density, $n_{\mathrm{sc}}$ is the density of storage centres, $\sigma$ is the optical cross-section for photostimulation and $I_{\mathrm{exc}}$ is the intensity of the laser photons (equivalent to the density of laser photons $n_{\mathrm{exc}}$ multiplied 
by the speed of light $c$ ).

$$
\frac{d n_{\mathrm{em}}(\mathbf{r})}{d t}=n_{\mathrm{sc}}(\mathbf{r}) \sigma I_{\mathrm{exc}}(\mathbf{r})
$$

Once the PSL photons have been emitted they will undergo the same random scattering events in the imaging plate as the laser photons, and are again followed until they are absorbed or leave from the front or rear surface. In this case the mean scattering length, $\mu$, anisotropy coefficient, $g$, and the optical absorption coefficient, $\gamma$, can have different values because of the different wavelengths for the laser $(\sim 630 \mathrm{~nm})$ and PSL photons $(\sim 390 \mathrm{~nm})$.

For PSL light the probability that a packet will leave the plate is important since this is what is detected. The probability of escape is found by assuming a set number of PSL photon packets are emitted from a certain depth in the plate. The photon packets which are emitted are followed until they leave the plate and the surface they leave from is recorded. The initial angular direction, $\theta$, of the packet is assumed to be isotropic with the use of a normalised phase function $p(\theta)=\frac{1}{4 \pi}$. Following the Monte Carlo technique described previously the probability distribution function $P(\theta)$ is found by integrating $p(\theta)$ over a sphere.

$$
P(\theta)=\frac{1}{2}(1-\cos \theta)
$$

Replacing $P(\theta)$ with a random number $R_{\theta}$ and rearraging for $\theta$ leads to the initial angular direction being randomly generated by equation 3.24.

$$
\theta=\cos ^{-1}\left(1-2 R_{\theta}\right)
$$

Reflection of the PSL photons at surfaces is again taken into account using Fresnel's reflection equation (equation 3.21). Photons which are transmitted at the interface are recorded and added to the total number of photons leaving the plate from each surface. The number of photons transmitted is given by $T=1-R$ 
The probability of escape is then the number of photons leaving a surface divided by the total number of photons emitted. This is calculated for all $z$ so that a probability versus depth in the plate, $P(z)$, can be determined.

\subsection{Line spread and modulation transfer functions}

As stated in section 2.4 the modulation transfer function is an important measure of the spatial resolution. This is determined computationally by simulating the line spread function and then Fourier transforming to find the MTF. Figure 3.7 shows graphically how the LSF is determined.

A simulation of the LSF can be determined by applying equation 3.22, for which the density profiles of the laser photons (as discussed in section 3.8) and storage centres must be known. The concentration of storage centres $n_{\mathrm{sc}}$ in the $z$ direction depends on the x-ray attenuation coefficient $\eta$, as shown in equation 3.25 .

$$
n_{\mathrm{sc}}(x, y, z)=n_{\mathrm{sc}}(x, y, 0) \exp (-\eta z)
$$

As with the intensity distributions (section 3.8), the imaging plate is split up into a number of voxels each of which contains a set laser photon density. Additionally, a matrix of storage centre densities is created with the use of equation 3.25 , the dimension of the matrix reflects the thickness of the plate and

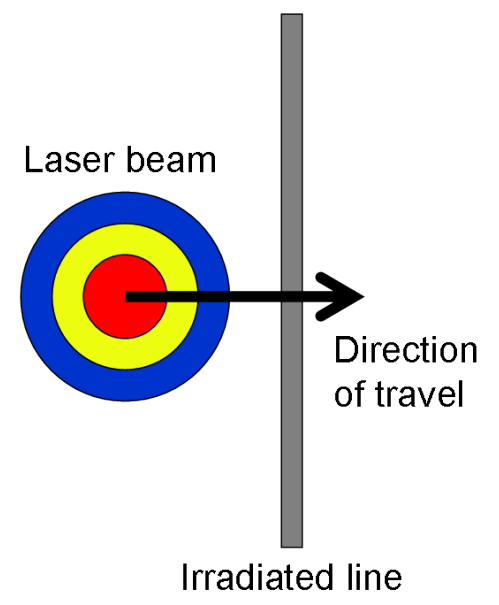

Figure 3.7: Conceptual picture showing how the LSF is determined. 
the length of the line. To simplify the simulation it was assumed that the laser was fixed and the line of irradiated centres was scanned across it. After scanning an amount $\Delta x$, the number of storage centres in the line (and the storage centre density matrix) will have decreased due to recombination and the consequent emission of PSL photons. The new storage centre density $n_{\mathrm{sc}}^{\prime}$ is given by equation 3.26 where $I_{\text {exc }}$ is the intensity of the laser photons, $\sigma$ the optical cross-section for photostimulation, and $t=\frac{\Delta x}{v_{\text {scan }}}$ is the time the laser was applied at each position.

$$
n_{\mathrm{sc}}(x, y, z)^{\prime}=n_{\mathrm{sc}}(x, y, z) \exp \left(-I_{\mathrm{exc}} t \sigma\right)
$$

The LSF is determined by equation 3.27 which calculates the response of the plate for a narrow line (width of $2 \mu \mathrm{m}$ ) containing a certain initial storage centre density, $n_{s c}$ laser photon density, $n_{\mathrm{exc}}$, and probability of escape, $P(z)$.

$$
L S F(x)=\sigma c \int_{z=0}^{d} d z P(z) \exp (-\eta z) \int_{y=-\infty}^{\infty} d y n_{\mathrm{exc}}(x, y, z) n_{\mathrm{sc}}(x, y, z)^{\prime}
$$

The integrals are over the thickness of the plate, $z=0$ to $d$ and length of the irradiated line $y=-\infty$ to $\infty$. Since the simulations are discrete in nature, equation 3.27 is changed from a continuous to a discrete equation by changing the integrals to sums and limiting the range of $y$ to between $-\frac{\text { lgth }}{2}$ and $\frac{\text { lgth }}{2}$, where lgth is the (discrete) length of the line.

$$
L S F(x)=\sigma c \sum_{z=0}^{d} \Delta z P(z) \exp (-\eta z) \sum_{y=-\frac{l g t h}{2}}^{\frac{l g t h}{2}} \Delta y n_{\mathrm{exc}}(x, y, z) n_{\mathrm{sc}}(x, y, z)^{\prime}
$$

To determine the MTF, a fast Fourier transform is applied to the results of equation 3.28 using MATLAB's fft function, and is then normalised at a spatial frequency of $\nu=0$.

The resolution is defined, following the industry norm, as the value at which 
the MTF falls to 0.2 and the sensitivity $(S)$ can be found using equation 3.29.

$$
S=\frac{\int_{-\infty}^{\infty} L S F(x) d x}{n_{\mathrm{sc}}(x, y, 0)}
$$





\section{Chapter 4}

\section{Scattering Simulation Results}

\subsection{Introduction}

This chapter shows simulation results obtained by varying different imaging plate properties. In simulating the read-out of an imaging plate there are many different parameters involved and a very large number of calculations could be done. To examine the trends, just a limited subset have been selected whilst keeping the remainder fixed at values which are representative of real XRSP materials and were determined with guidance from the literature [17] [18]. The simulation parameters which are held constant are shown in table 4.1. In all simulations the $x$ and $y$ plate dimensions (typically $3 \mathrm{~mm}$ to $20 \mathrm{~mm}$ ) are assumed to be much larger than the thickness of the plate, meaning all photons exit from either the front or rear surface of the plate. It was also assumed, unless otherwise stated, that the absorption coefficient and scattering length are the same for both laser and PSL photons although the programming makes allowance for these to be different. This could be important for small particles, where a Rayleigh-like dependence of scattering coefficient on wavelength could introduce a difference of $\left(\lambda_{\text {laser }} / \lambda_{\mathrm{PSL}}\right)^{4}$ between the two. 


\begin{tabular}{ccc}
\hline Parameter & Description & Value \\
\hline$\sigma$ & Optical cross-section for photostimulation & $8.85 \times 10^{-20} \mathrm{~m}^{2}$ \\
$n_{\mathrm{sc}}$ & Initial density of storage centres & $1.0 \times 10^{21} \mathrm{~m}^{-3}$ \\
$v_{\mathrm{scan}}$ & Laser scanning speed & $1.0 \times 10^{7} \mu \mathrm{ms}^{-1}$ \\
$\Delta x$ & Laser step size & $5.0 \mu \mathrm{m}$ \\
$E_{\text {photon }}$ & Energy of laser photons & $3.142 \times 10^{-19} \mathrm{~J}$ \\
$\Delta \rho$ & Packet step size & $1.0 \mu \mathrm{m}$ \\
$N_{\text {pkt }}$ & Number of packets & 1000000 \\
$n_{1}$ & Refractive index of medium & 1.498 \\
$n_{2}$ & Refractive index of surroundings & 1.0 \\
$Z$ & Plate thickness & $200.0 \mu \mathrm{m}$ \\
\hline
\end{tabular}

Table 4.1: Simulation parameters held constant in all simulations.

\subsection{Dependence on scattering length}

The scattering length was varied from a large scattering length $(20000 \mu \mathrm{m})$ corresponding to a relatively transparent material to a small scattering length (2 $\mu \mathrm{m})$ corresoponding to a highly opaque material. Parameters for these simulations can be seen in table 4.2. A $g$ of 0.5003 refers to particles with a radius of approximately $100 \mathrm{~nm}$ which is appropriate for fluorozirconate glass but significantly smaller than found in BaFBr imaging plates.

Intensity distribution profiles of these simulations can be seen in figure 4.1. In decreasing the scattering length (decreasing the transparency) the spread of the laser photons increases until the scattering length is smaller than the plate thickness where the spread decreases. For large scattering lengths, figure $4.1(\mathrm{a}),(\mathrm{b})$, most of the laser photons pass straight through the plate with

\begin{tabular}{ccc}
\hline Parameter & Description & Value \\
\hline$\mu$ & Mean scattering length & $2-20000 \mu \mathrm{m}$ \\
$\gamma$ & Absorption coefficient & $0.001 \mu \mathrm{m}^{-1}$ \\
$g_{1}$ & Anisotropy coefficient for laser light & 0.5003 \\
$g_{2}$ & Anisotropy coefficient for PSL light & 0.7602 \\
$P$ & Laser power & $0.1 \mathrm{~mW}$ \\
$w$ & Laser variance & $20 \mu \mathrm{m}$ \\
$\eta$ & X-ray attenuation coefficient & $0.016 \mu \mathrm{m}^{-1}$ \\
\hline
\end{tabular}

Table 4.2: Parameters for simulations with varying scattering lengths. 


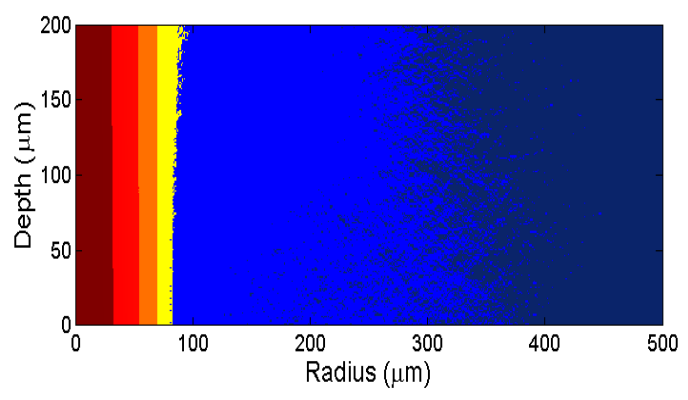

(a) $\mu=20000 \mu \mathrm{m}$

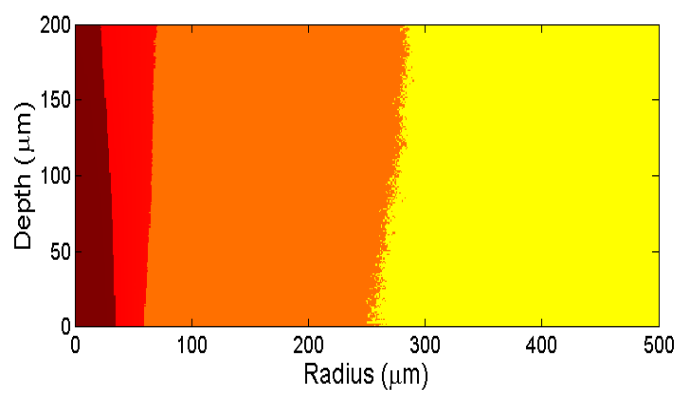

(c) $\mu=200 \mu \mathrm{m}$

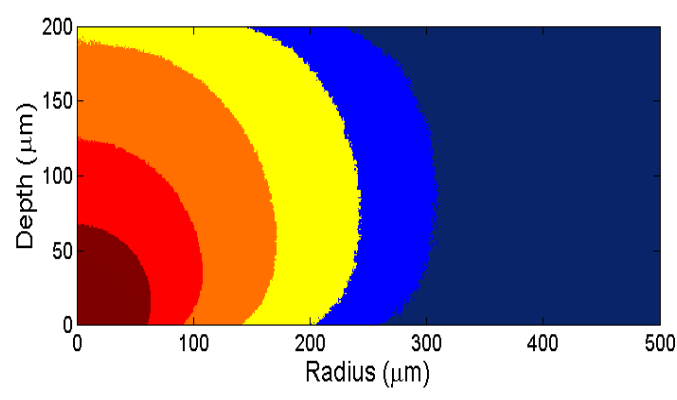

(e) $\mu=2 \mu \mathrm{m}$

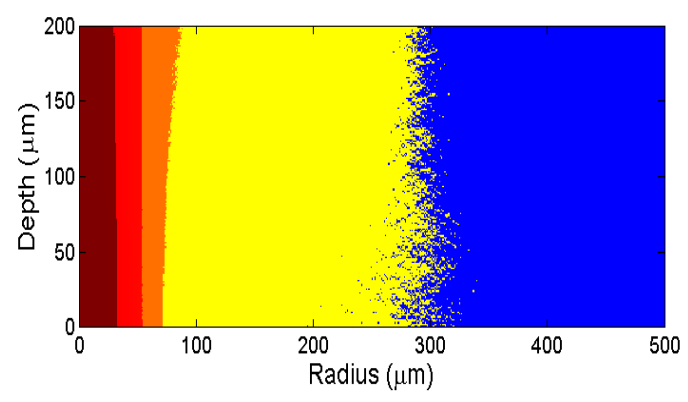

(b) $\mu=2000 \mu \mathrm{m}$

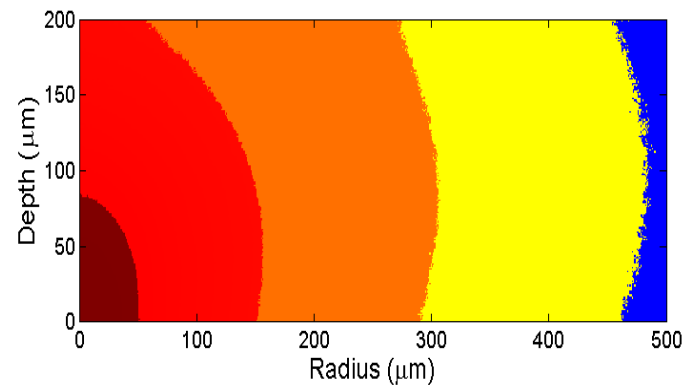

(d) $\mu=20 \mu \mathrm{m}$

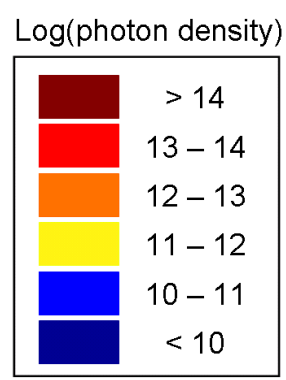

(f) key

Figure 4.1: (a-e) Intensity distributions profiles for large particles with varying scattering lengths as shown. (f) Logarithmic scale of the photon densities.

only a small number of packets being scattered. The initial increase in spread as the scattering length decreases is due to a larger number of packets being scattered. At very small scattering lengths, figure 4.1(e), the spread decreases due to packets being scattered more often and are not able to travel as far in the plate before exiting or being absorbed.

The probability of escape of PSL photons generated at a depth $z$ from the front surface of the plate is shown in figure 4.2. For small scattering lengths and shallow depth, there is a high probability that photon packets which set off in the direction of the rear face will be back-scattered and eventually leave from the front surface of the plate. Increasing the scattering length causes the prob- 


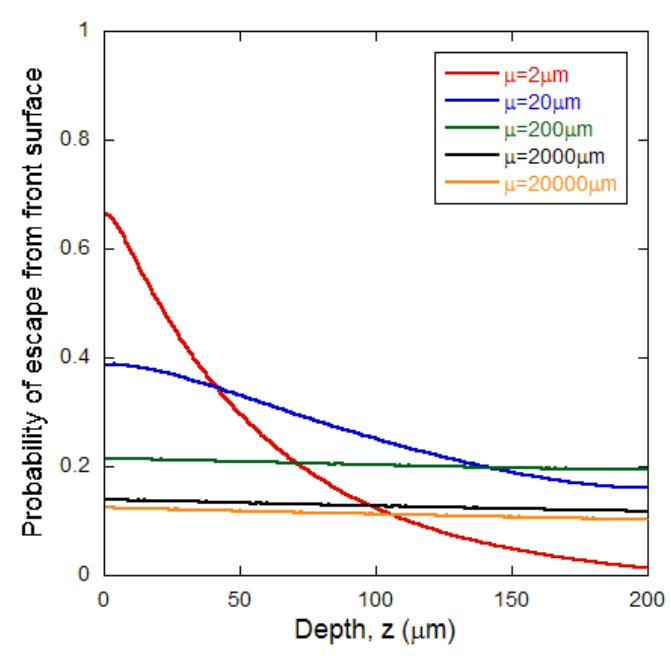

Figure 4.2: Probability of escape from the front surface of PSL photons generated at a depth $z$ in the plate. The various scattering lengths are as shown.

ability of escape to decrease from shallow depths. At large scattering lengths it becomes equally probable for a photon generated at the rear of the plate to escape from the front surface as a photon generated at the front of the plate. The flattening of the probability of escape is caused by the packets travelling greater distances in the plate - packets can bounce back and forth several times through the thickness of the plate before escaping or being absorbed.

Figure 4.3 shows the LSF and MTF for photons escaping from the front surface of the plate. The height of the LSF decreases with increasing scattering length

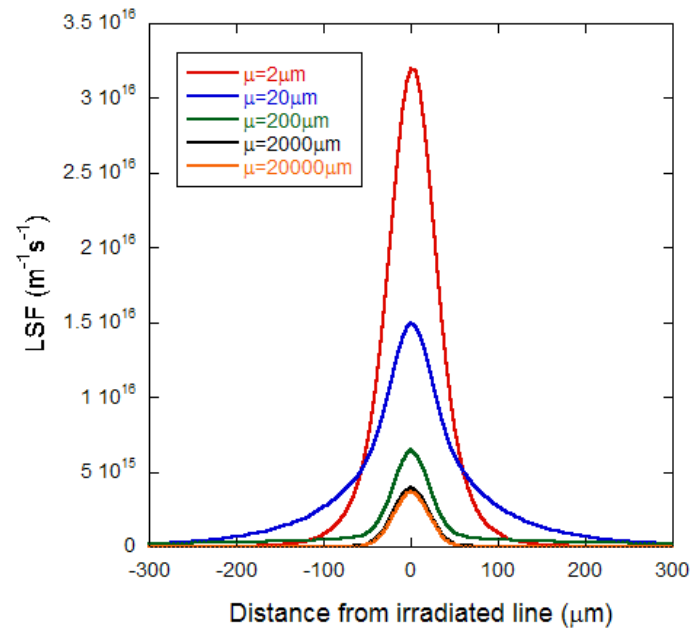

(a)

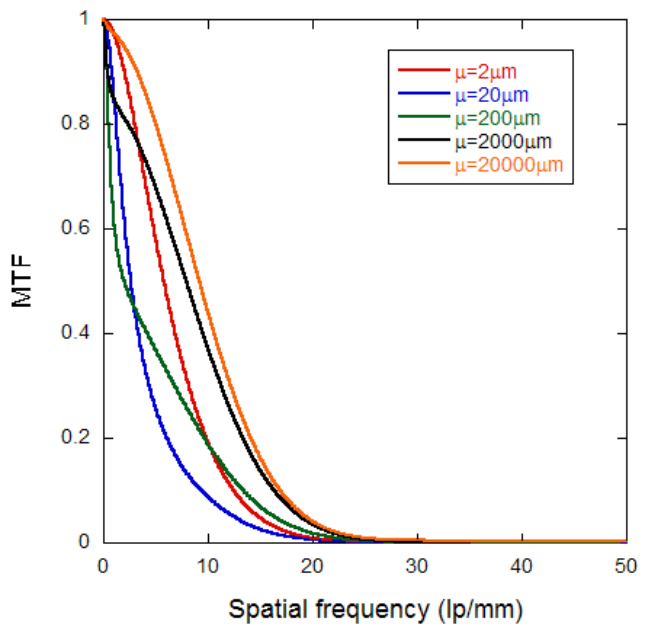

(b)

Figure 4.3: (a) LSF's and (b) MTF's for photons escaping from the front surface with varying scattering lengths as shown. 
which is caused by both the decrease in probability of escape and the laser photons travelling shorter distances in the plate. For large scattering lengths the LSF's show a bimodal nature with the narrow central peak being in essence the laser beam profile whilst the long tails are caused by scattering. These extend out to large lateral distances for scattering lengths of 2000 and 20000 micrometres (approximately $15 \mathrm{~mm}$ for $\mu=2000 \mu \mathrm{m}$ and $20 \mathrm{~mm}$ for $\mu=20000 \mu \mathrm{m}$ ) which cannot be seen on the graph. The bimodal nature is clearly seen in figure $4.3(\mathrm{~b})$ for a scattering length of $200 \mu \mathrm{m}$. For small scattering lengths the LSF curves show no scattering tails, instead they show a scattering-induced broadening of the Gaussian distribution of the laser beam which can also be seen in figure 4.1(e).

Taking the "resolution" to be at a MTF equal to 0.2 following industry practice, the curves show that by decreasing the scattering length the resolution at first decreases from $\sim 15 \mathrm{lp} / \mathrm{mm}$ to a minimum of $\sim 6 \mathrm{lp} / \mathrm{mm}$ and then increases again for very short scattering lengths, but not to the same value as for large scattering lengths. Large scattering lengths also correspond to a bimodal nature for the MTF. The narrow component peak at low frequencies is caused by the extended scattering tails and the broader component curve at higher fre-

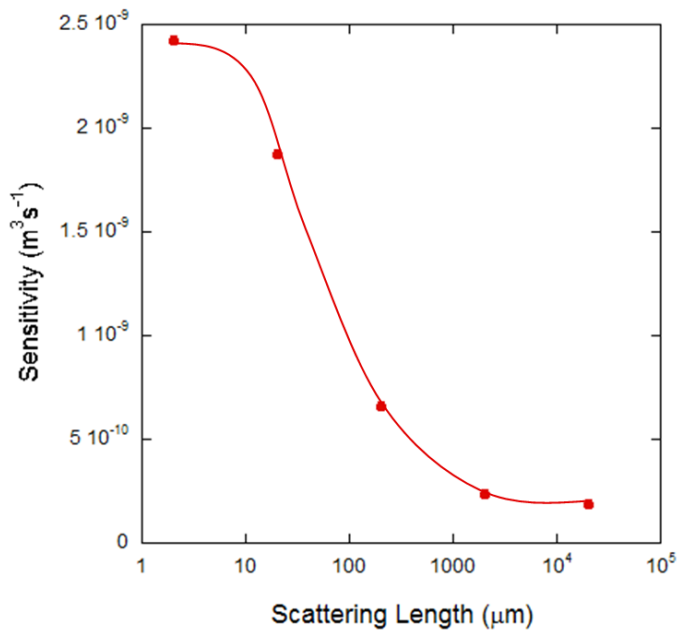

(a)

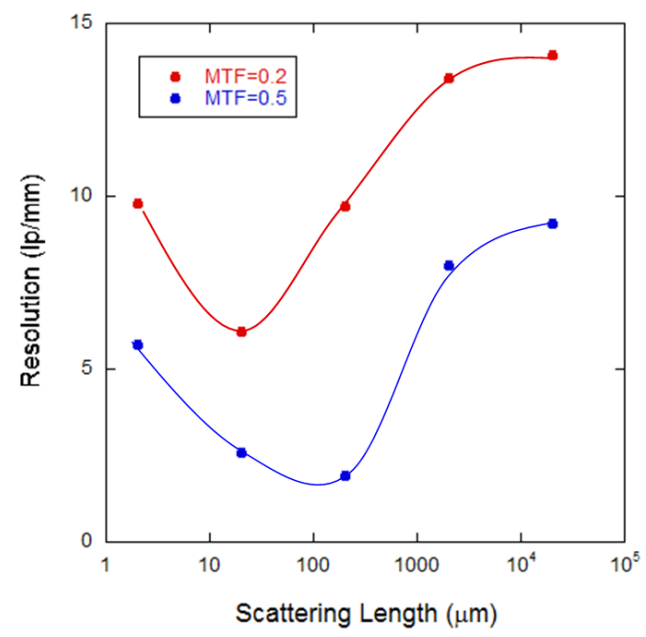

(b)

Figure 4.4: (a) Sensitivity and (b) Resolution plots for photons escaping from the front surface with varying scattering lengths. The lines are guides for the eye. 
quencies is due to the laser beam width.

Figure 4.4 shows the sensitivity and resolution plots that are derived from the LSF's and MTF's as explained in section 3.10. Increasing the scattering length decreases the sensitivity as there more photons passing straight through the plate which are unable to stimulate the irradiated centres. The resolution is determined for an MTF of both 0.2 (red dots on figure 4.4(b)) and 0.5 (blue dots), as the shape of the MTF changes. The highest resolution comes from a transparent material with a large scattering length and the minimum resolution occurs at $20 \mu \mathrm{m}$ for an MTF of 0.2 and $200 \mu \mathrm{m}$ for an MTF of 0.5. An increase in resolution comes at a cost of a decrease in sensitivity.

In most commercial applications only the photons leaving from the front surface of the plate are recorded. However, photons can also leave from the rear surface of the plate and the effect rear face imaging has on the resolution and sensitivity has been investigated.

The probability of escape for photons exiting from the rear surface of the plate (figure 4.5) again shows that the probability decreases with increasing scattering length. The graph shows the same trends as for escape from the front surface but in the opposite direction.

Graphs of the LSF and MTF for photons escaping from the rear of the plate are

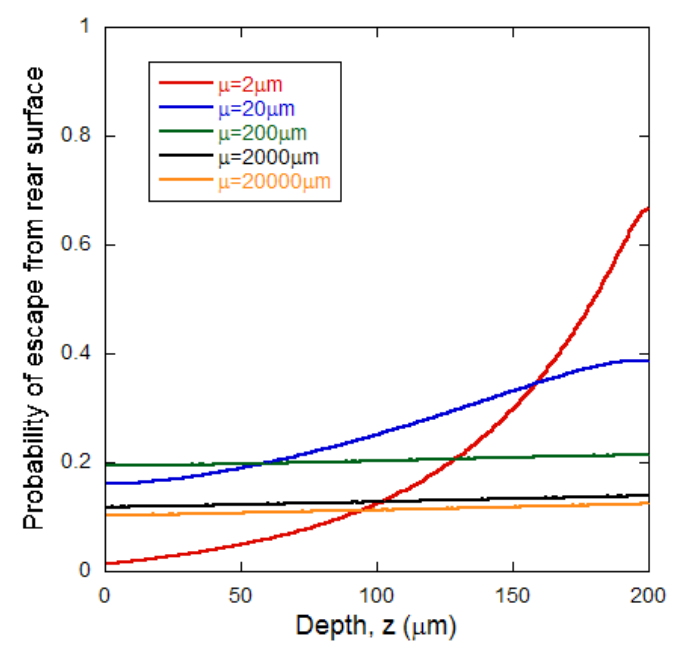

Figure 4.5: Probability of escape from the rear surface of PSL photons generated at a depth $z$ in the plate. The various scattering lengths are as shown. 


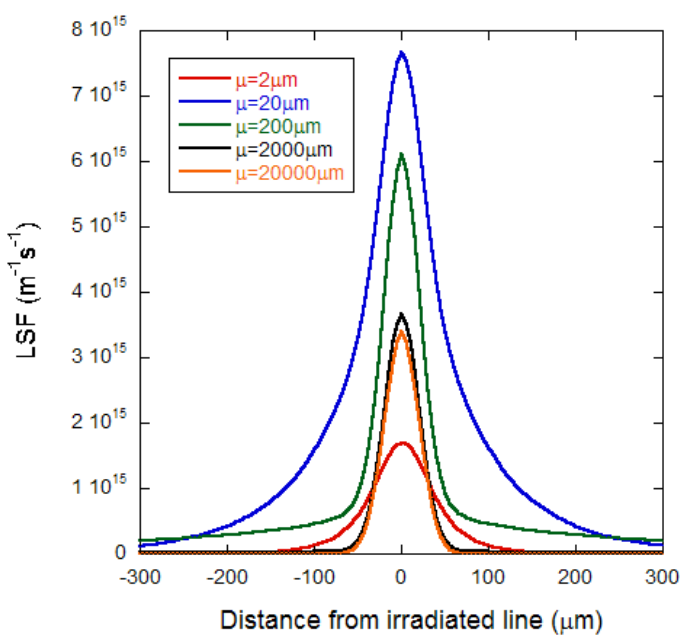

(a)

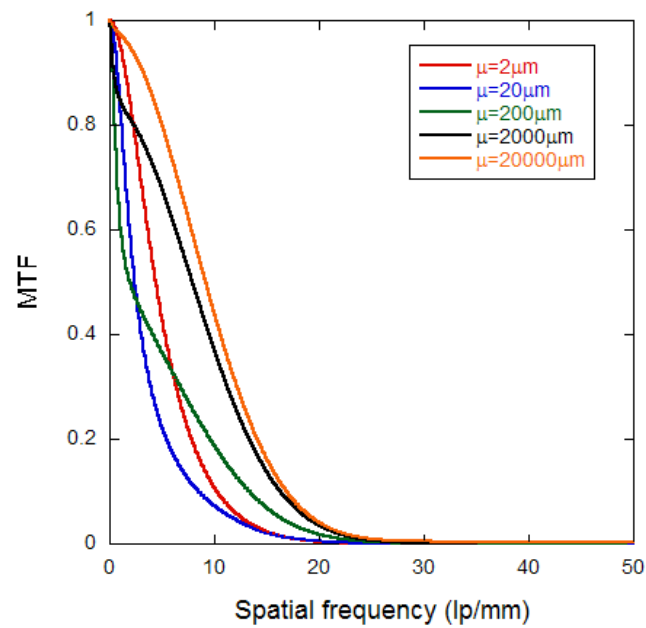

(b)

Figure 4.6: (a) LSF's and (b) MTF's for photons escaping from the rear surface with varying scattering lengths as shown.

shown in figure 4.6. LSF curves for large scattering lengths are similar to those for photons escaping from the front surface which is because the main laser beam passes straight through the plate and the probability for escape from the rear surface is the same as for the front. At very small scattering lengths (red curve on figure 4.6(a)) the height of the LSF is greatly reduced due to the probability of photons leaving from the rear of the plate being much smaller compared to those leaving from the front. This implies that for plates with a

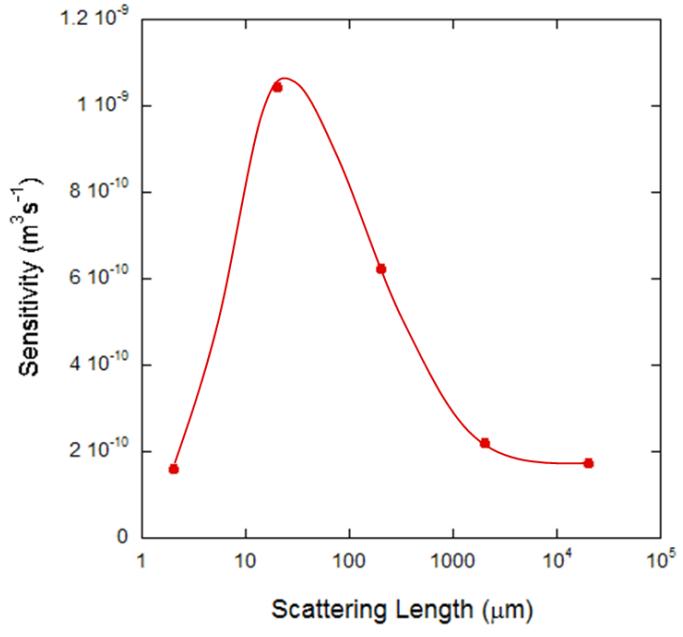

(a)

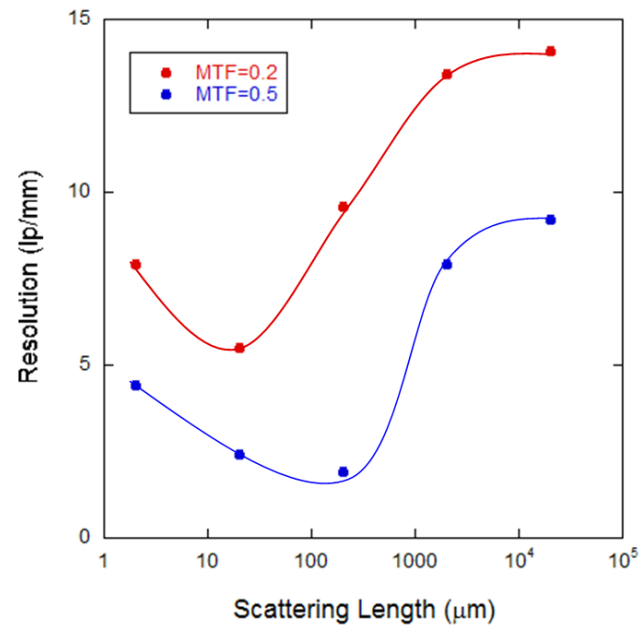

(b)

Figure 4.7: (a) Sensitivity and (b) Resolution plots for photons escaping from the rear surface with varying scattering lengths. The lines are guides for the eye. 
small scattering length rear face imaging is not a viable option.

Resolution and sensitivity plots are shown in figure 4.7. These show the same trends as for photons emitted from the front surface of the plate with the sensitivity being reduced for small scattering lengths. The resolution is minimum at $\mu=20 \mu \mathrm{m}$ for an MTF equal to 0.2 and at $\mu=200 \mu \mathrm{m}$ for an MTF equal to 0.5 and the sensitivity is at a maximum for a scattering length of $20 \mu \mathrm{m}$.

\subsection{Variations in absorption coefficient}

The absorption coefficient for both stimulating and PSL light was varied from no absorption $\left(\gamma=0.0 \mu \mathrm{m}^{-1}\right)$ to strong absorption $\left(\gamma=0.05 \mu \mathrm{m}^{-1}\right.$; a half length of approximately $14 \mu \mathrm{m})$. The half length is the length at which half of the initial intensity has been absorbed. Parameters for these simulations are presented in table 4.3 .

The intensity distributions for these simulations are shown in figure 4.8. Increasing the absorption coefficient decreases the spread of the photons because more photons are absorbed over a given distance and so the photon cloud is unable to spread as far into the plate. For absorption coefficients for $0.005 \mu \mathrm{m}^{-1}$ and above the main beam of the laser does not pass all the way through the plate, instead is attenuated before it reaches the rear surface.

Figure 4.9 shows the probability of escape of PSL photons from the front surface of the plate. The probability of escape decreases with increasing absorp-

\begin{tabular}{ccc}
\hline Parameter & Description & Value \\
\hline$\mu$ & Mean scattering length & $200 \mu \mathrm{m}$ \\
$\gamma$ & Absorption coefficient & $0.0-0.05 \mu \mathrm{m}^{-1}$ \\
$g_{1}$ & Anisotropy coefficient for laser light & 0.5003 \\
$g_{2}$ & Anisotropy coefficient for PSL light & 0.7602 \\
$P$ & Laser power & $0.1 \mathrm{~mW}$ \\
$w$ & Laser variance & $20 \mu \mathrm{m}$ \\
$\eta$ & X-ray attenuation coefficient & $0.016 \mu \mathrm{m}^{-1}$ \\
\hline
\end{tabular}

Table 4.3: Parameters for simulations with varying absorption coefficients. 


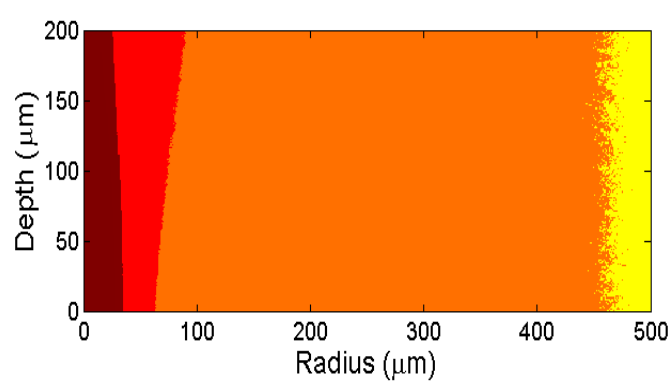

(a) $\gamma=0.0 \mu \mathrm{m}^{-1}$

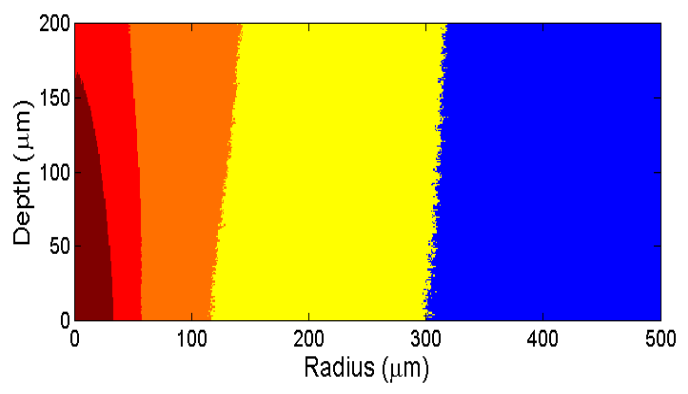

(c) $\gamma=0.005 \mu \mathrm{m}^{-1}$

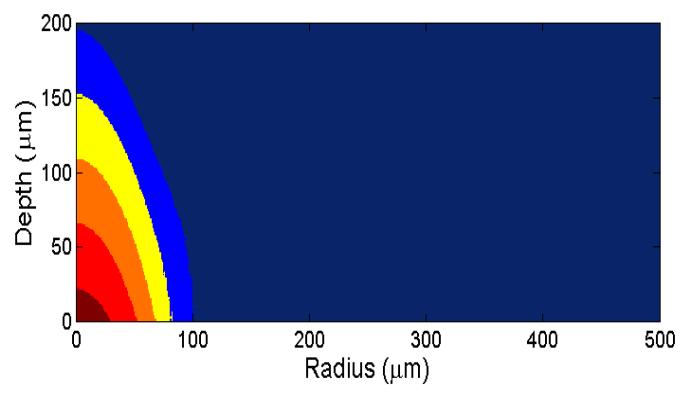

(e) $\gamma=0.05 \mu \mathrm{m}^{-1}$

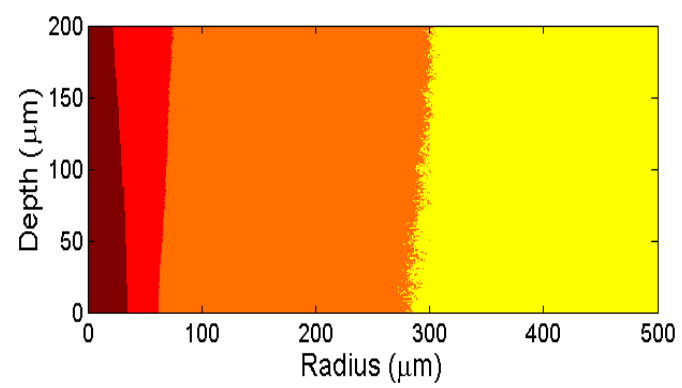

(b) $\gamma=0.001 \mu \mathrm{m}^{-1}$

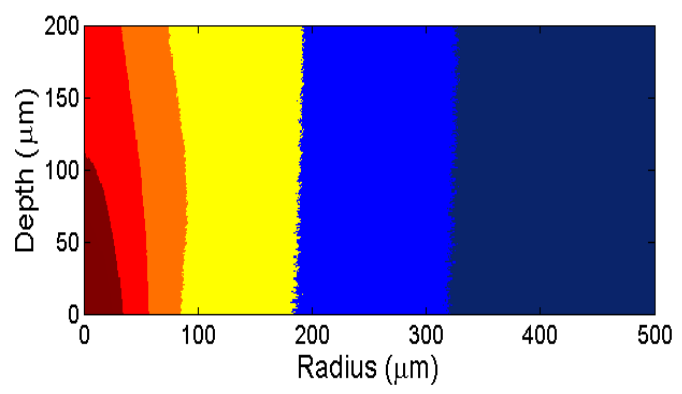

(d) $\gamma=0.01 \mu \mathrm{m}^{-1}$

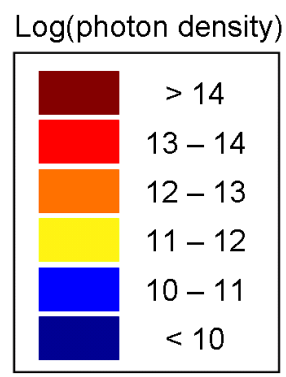

(f) key

Figure 4.8: (a-e) Intensity distribution profiles for varying absorption coefficients as shown. (f) Logarithmic scale of the photon densities.

tion because more photons are being absorbed and less are escaping from the plate. For small absorption coefficients the probability of escape of a photon generated at the rear of the plate is approximately the same as for a photon generated at the front. As the absorption coefficient increases it becomes less likely for a photon generated at the rear of the plate to escape from the front surface than it is for a photon generated at the front.

The LSF and MTF for varying absorption coefficients can be seen in figure 4.10. Increasing the absorption decreases the LSF amplitude as there are fewer nonabsorbed stimulating photons in the plate and fewer PSL photons escape from the plate. The scattering tails clearly seen on the red curve in figure 4.10(a) 


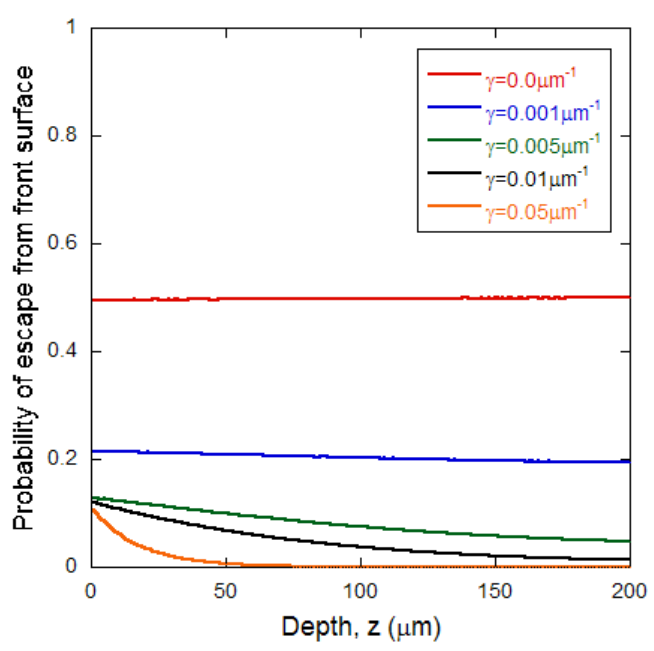

Figure 4.9: Probability of escape from the front surface of PSL photons generated at a depth $z$ in the plate. The various absorption coefficients are as shown.

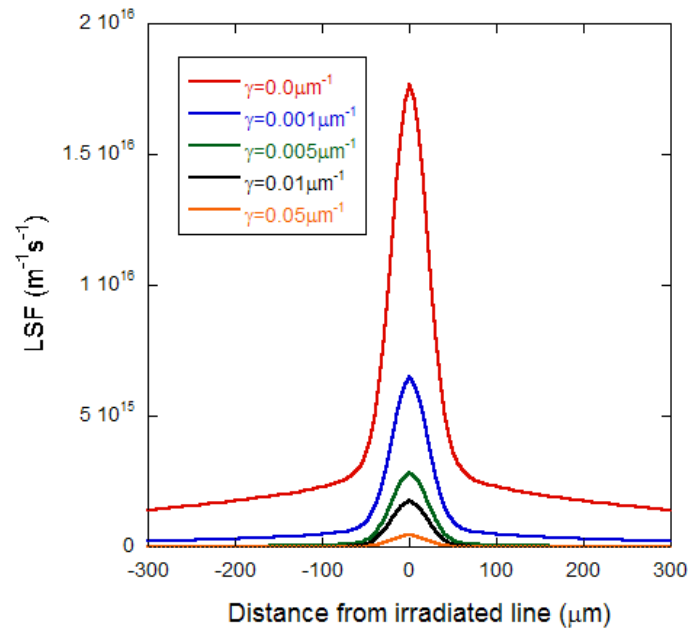

(a)

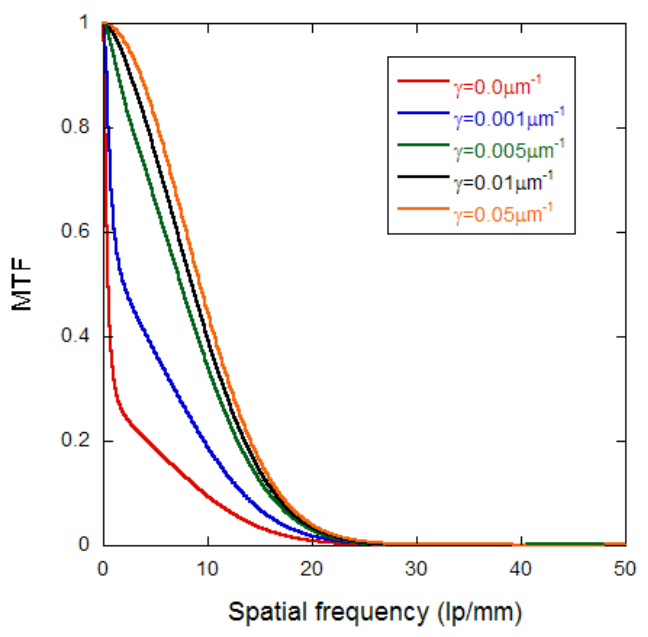

(b)

Figure 4.10: (a) LSF's and (b) MTF's for photons escaping from the front surface with varying absorption coefficients as shown.

decrease with increasing absorption. The width of the MTF increases with increasing absorption as the spread caused by scattering is decreased and the bimodal nature seen at low absorption coefficients disappears due to the reduction in scattering tails.

Figure 4.11 shows the sensitivity and resolution plots for varying the absorption coefficient. The sensitivity decreases sharply with increasing absorption coefficient which is caused by photons being increasingly absorbed in the volume of the plate and fewer escaping. The resolution increases with increasing 


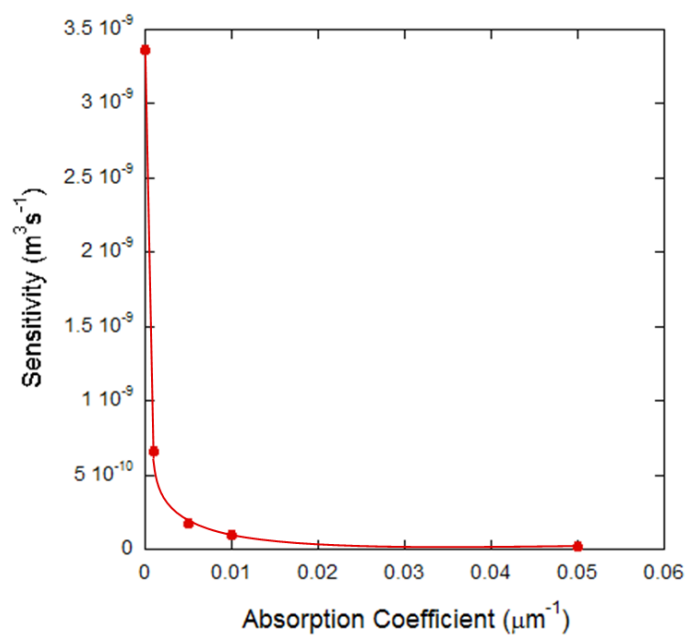

(a)

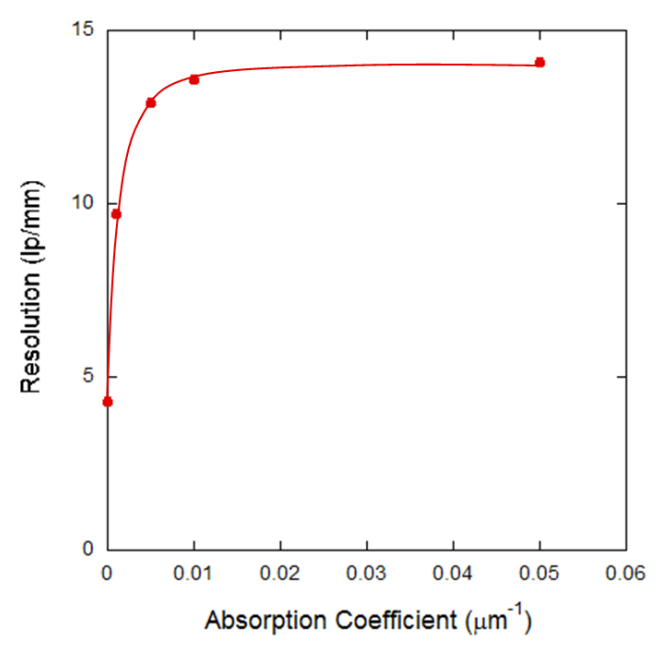

(b)

Figure 4.11: (a) Sensitivity and (b) Resolution plots for photons escaping from the front surface with varying absorption coefficients. The lines are guides for the eye.

absorption coefficient as the spread of the laser photons is decreased.

\subsection{Adding selective absorption of the stimulating light}

By doping the glass ceramic with various additives the absorption properties can be selectively changed. One such material is cobalt fluoride which increases the absorption coefficient for red laser photons but has little effect on the absorption of blue-UV PSL photons [26]. To investigate this effect, simulations were run with absorption coefficients varying between $0.001 \mu \mathrm{m}^{-1}$ and $0.05 \mu \mathrm{m}^{-1}$ for red laser photons and a fixed absorption coefficient of $0.001 \mu \mathrm{m}^{-1}$ for PSL photons. The parameters for these simulations can be found in table 4.4 .

Intensity distributions of the laser photons are the same as those shown in figure 4.8 as the PSL photons have no influence on the scattering of the laser photons.

The probability of escape of PSL photons is not shown as it does not change 


\begin{tabular}{ccc}
\hline Parameter & Description & Value \\
\hline$\mu$ & Mean scattering length & $200 \mu \mathrm{m}$ \\
$\gamma_{1}$ & Absorption coefficient laser photons & $0.001-0.05 \mu \mathrm{m}^{-1}$ \\
$\gamma_{2}$ & Absorption coefficient PSL photons & $0.001 \mu \mathrm{m}^{-1}$ \\
$g_{1}$ & Anisotropy coefficient for laser light & 0.5003 \\
$g_{2}$ & Anisotropy coefficient for PSL light & 0.7602 \\
$P$ & Laser power & $0.1 \mathrm{~mW}$ \\
$w$ & Laser variance & $20 \mu \mathrm{m}$ \\
$\eta$ & X-ray attenuation coefficient & $0.016 \mu \mathrm{m}^{-1}$ \\
\hline
\end{tabular}

Table 4.4: Parameters for simulations with added absorption.

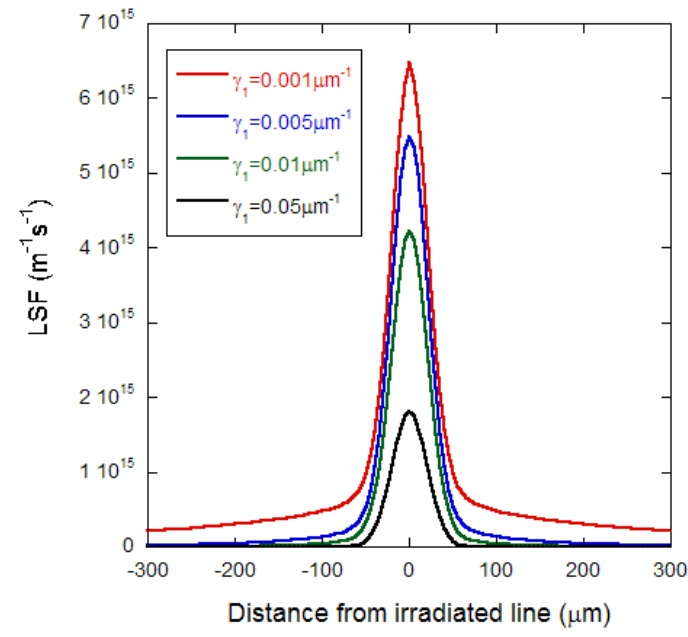

(a)

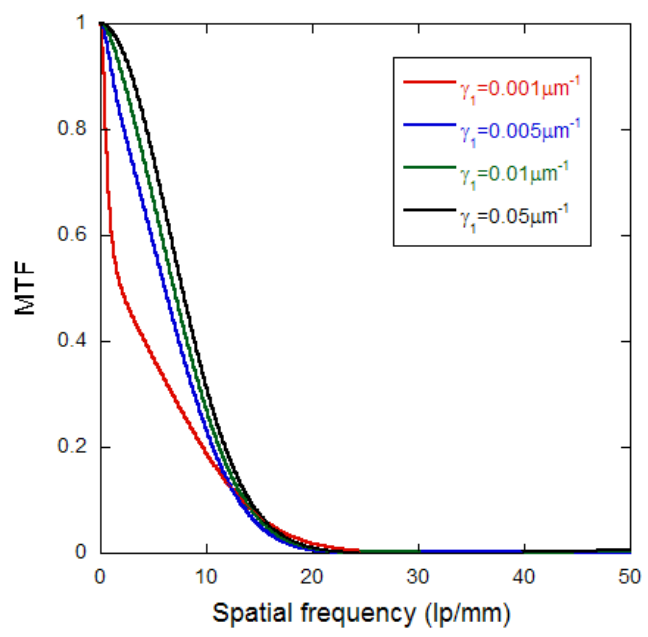

(b)

Figure 4.12: (a) LSF's and (b) MTF's for photons escaping from the front surface with various laser photon absorption coefficients as shown.

with variations in the absorption coefficient of laser photons.

Figure 4.12 shows the LSF and MTF for the various absorption coefficients. Increasing the absorption coefficient again decreases the amplitude of the LSF and increases the MTF width as seen in section 4.3. In varying the absorption coefficients for laser photons, the changes in the LSF's are compared to the results shown previously for increasing both absorption coefficients, and are reproduced here as figure 4.13 excluding the case of $\gamma_{1}=0.0 \mu \mathrm{m}^{-1}$. The amplitude of the LSF curves for each absorption coefficient increases for the case of added absorption of laser photons, except for $\gamma_{1}=0.001 \mu \mathrm{m}^{-1}$ which has the same parameters for both cases. The increase in height of the LSF curves (figure 4.12(a)) is because the probability of escape of PSL photons stays the 


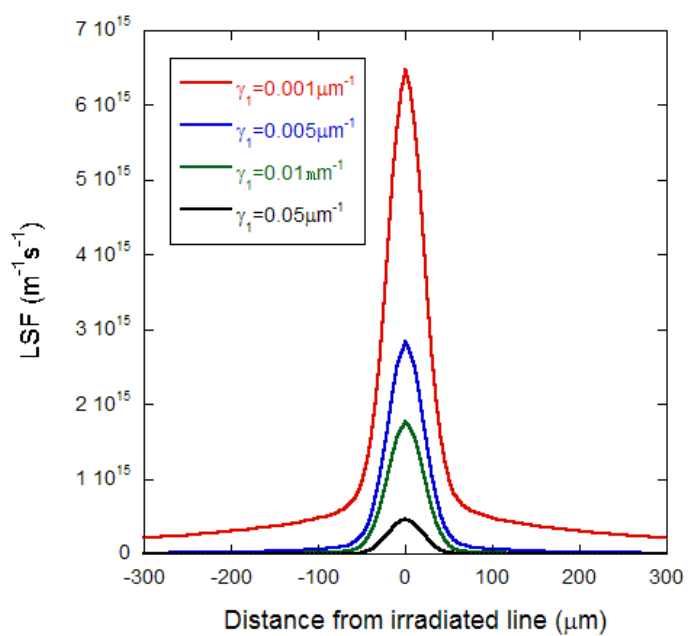

(a)

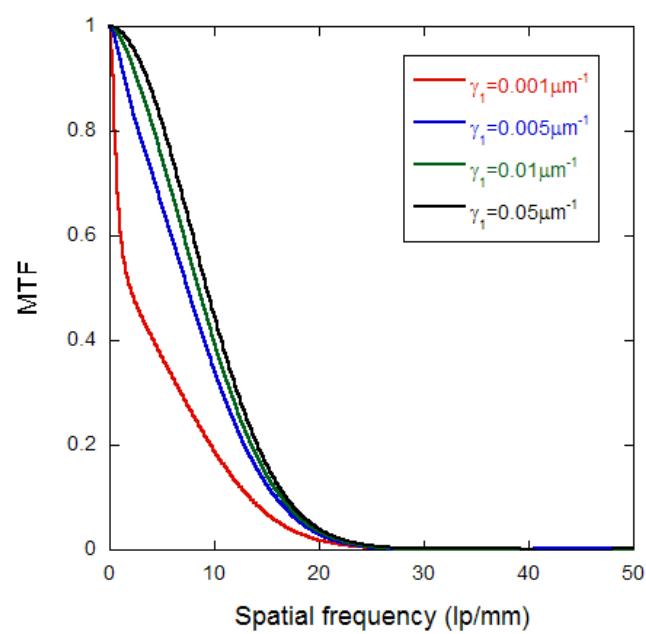

(b)

Figure 4.13: Reproduced (a) LSF's and (b) MTF's for photons escaping from the front surface with varying both absorption coefficients as shown. The LSF and MTF for an absorption coefficient of $\gamma=0.0 \mu \mathrm{m}^{-1}$ has been omitted.

same, so more PSL photons are escaping from the plate and being detected for a given laser photon absorption coefficient as compared to the previous results (figure 4.13(a)). The width of the MTF curves for added laser photon absorption decreases above $\gamma=0.001 \mu \mathrm{m}$ as compared to previous results. This leads to a slightly lower resolution (approximately $2 \mathrm{lp} / \mathrm{mm}$ ) in the case of selective absorption.

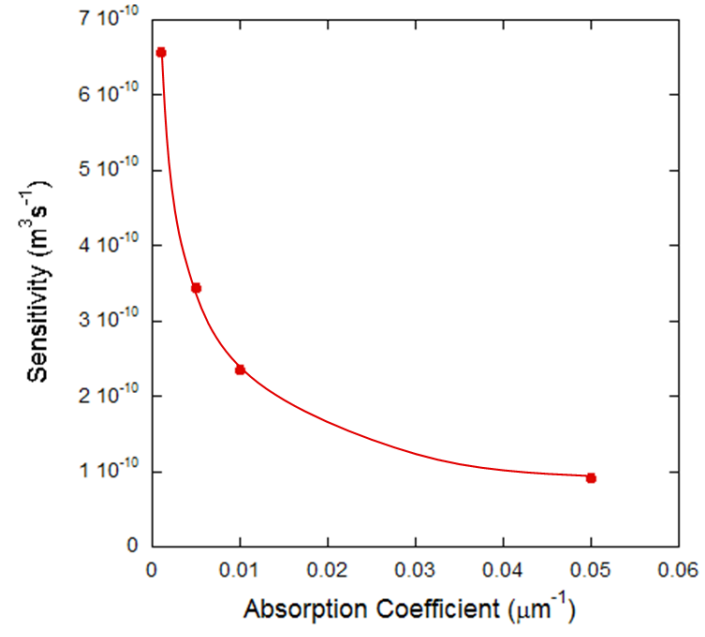

(a)

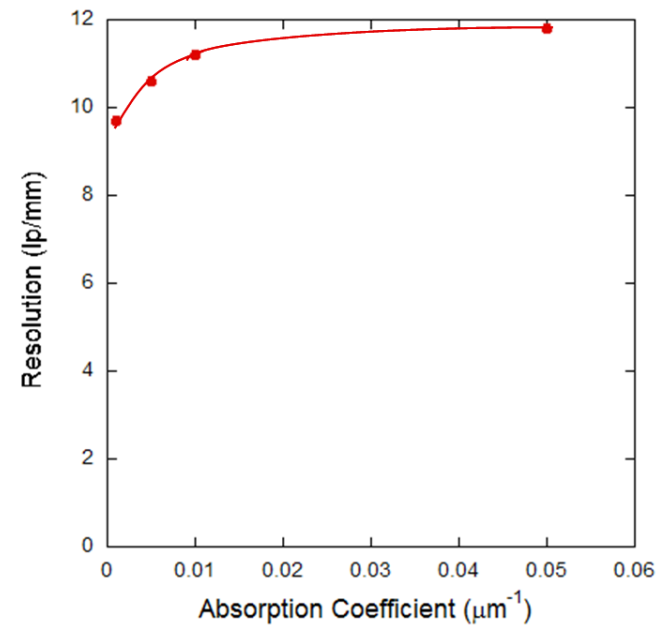

(b)

Figure 4.14: (a) Sensitivity and (b) Resolution plots for photons escaping from the front surface with varying laser photon absorption coefficients. The lines are guides for the eye. 
Figure 4.14 shows the resolution and sensitivity plots for adding absorption. Increasing the added absorption decreases the sensitivity and increases the resolution as was seen previously. When compared to the results in figure 4.11 the sensitivity is increased and resolution decreased for absorption coefficients above $0.001 \mu \mathrm{m}^{-1}$.

\subsection{Effect of particle size}

The effect particle size has on the resolution and sensitivity has been investigated. Generally, a variation in particle size will also affect the bulk scattering length for an actual sample, but for these simulations the scattering length describing the probable length between scattering events was assumed to be fixed. The parameters for these simulations are shown in table 4.5. Strictly what is being tested here is the dependence on the angular variation of the scattering, but the results should be indicative of the effect of particle size.

In the simulations, a change in the particle size affects the anisotropy coeffi-

\begin{tabular}{ccc}
\hline Parameter & Description & Value \\
\hline$\mu$ & Mean scattering length & $200 \mu \mathrm{m}$ \\
$\gamma$ & Absorption coefficient & $0.001 \mu \mathrm{m}^{-1}$ \\
$g_{1}$ & Anisotropy coefficient for laser light & $0.0048-0.9614$ \\
$g_{2}$ & Anisotropy coefficient for PSL light & $0.0126-0.9207$ \\
$P$ & Laser power & $0.1 \mathrm{~mW}$ \\
$w$ & Laser variance & $20 \mu \mathrm{m}$ \\
$\eta$ & X-ray attenuation coefficient & $0.016 \mu \mathrm{m}^{-1}$ \\
\hline
\end{tabular}

Table 4.5: Parameters for simulations with various particle sizes.

\begin{tabular}{ccccc}
\hline Particle size $(\mathrm{nm})$ & $g_{1}$ & $x_{1}$ & $g_{2}$ & $x_{2}$ \\
\hline 10 & 0.0048 & 0.1489 & 0.0126 & 0.2417 \\
100 & 0.5003 & 1.489 & 0.7602 & 2.417 \\
200 & 0.7838 & 2.918 & 0.8975 & 4.833 \\
1000 & 0.9614 & 14.89 & 0.9207 & 24.17
\end{tabular}

Table 4.6: Anisotropy coefficients $(g)$ and size parameters $(x)$ for various particle radii $(a)$. 
cient, $g$, and is different for laser and PSL photons due to their different wavelengths (633 $\mathrm{nm}$ and $390 \mathrm{~nm}$ respectively). The particle radius was varied from $10 \mathrm{~nm}$ to $1 \mu \mathrm{m}$ which corresponds to a variation in $g$ for the laser photons of $0.0048-0.9614$ and $0.0126-0.9207$ for PSL photons. Table 4.6 shows the different particles sizes and their corresponding anisotropy coefficients and size parameters for both laser $\left(g_{1}, x_{1}\right)$ and PSL $\left(g_{2}, x_{2}\right)$ photons. The values of $g$ were determined by calculating the average cosine of the Mie phase function for a given particle size, as described in section 2.2.3.

Intensity distributions for the various particle sizes are shown in figure 4.19.

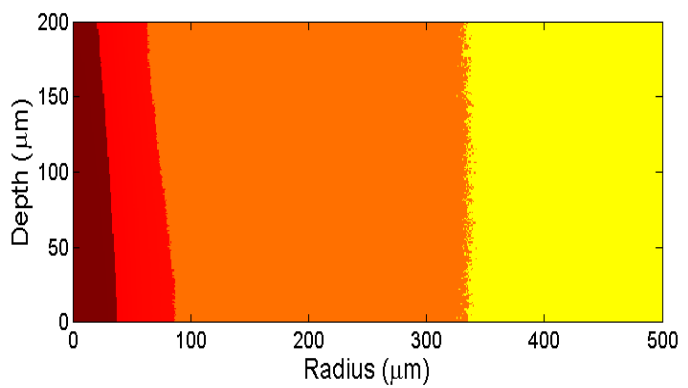

(a) $a=10 \mathrm{~nm}$

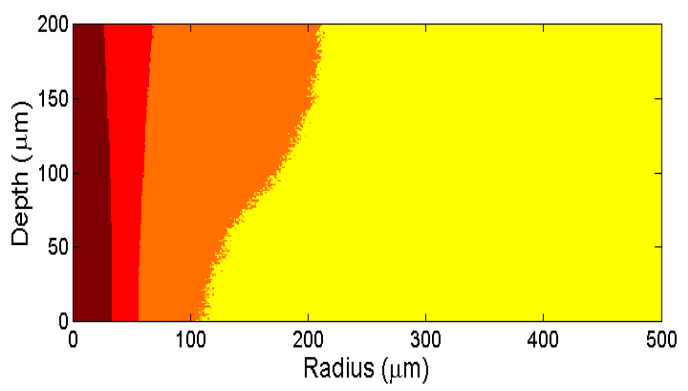

(c) $a=200 \mathrm{~nm}$

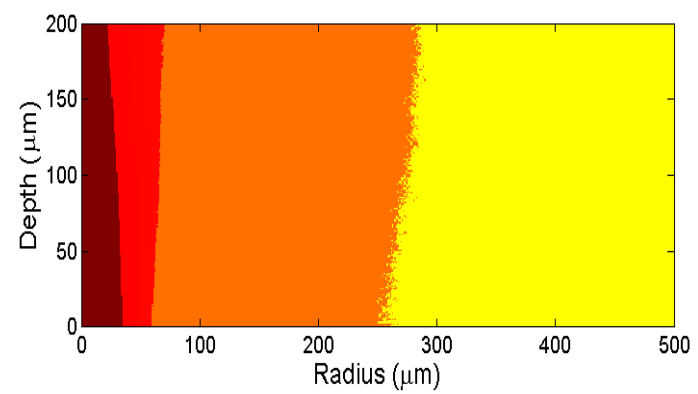

(b) $a=100 \mathrm{~nm}$

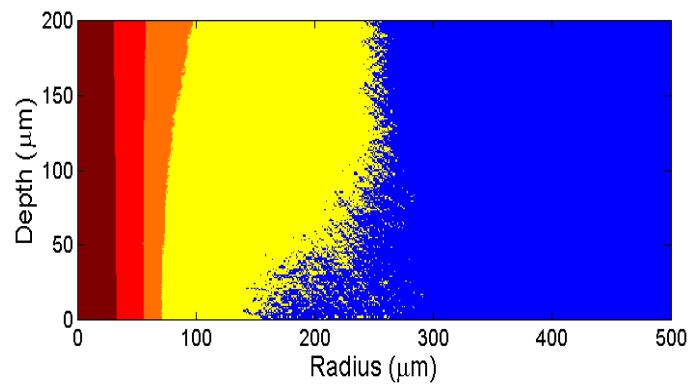

(d) $a=1000 \mathrm{~nm}$

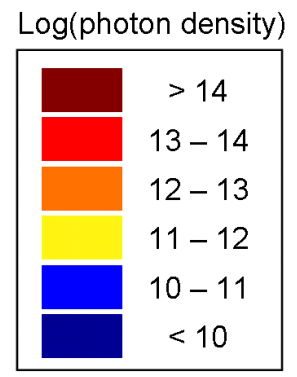

(e) key

Figure 4.15: (a-d) Intensity distribution profiles for various particle sizes as shown. (e) Logarithmic scale of the photon densities. 


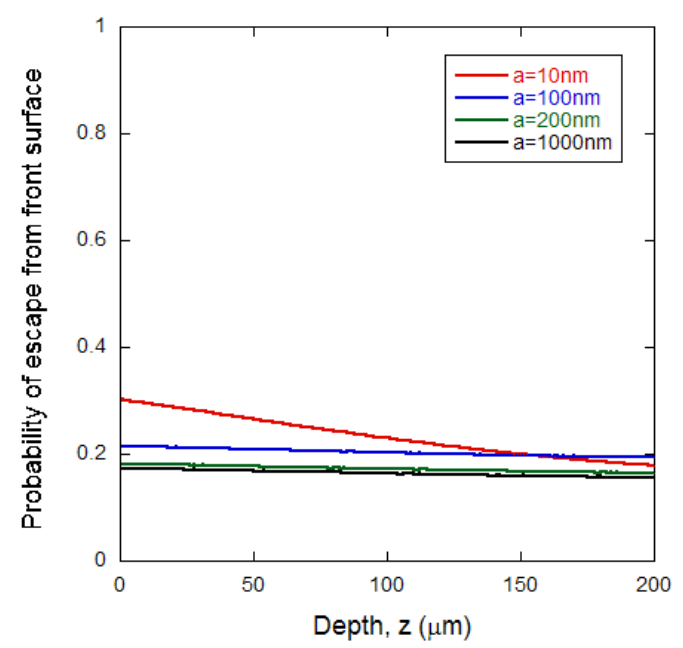

Figure 4.16: Probability of escape from the front surface of PSL photons generated at a depth $z$ in the plate. The various particle sizes are as shown.

Increasing the particle size decreases the lateral spread of the scattered laser photons as they are predominately scattered in the forwards direction and do not travel as far in the plate before escaping or being absorbed as for small particles. In the latter case of near isotropic scattering, the scattered photon density follows a near cylindrical symmetry. Increasing the particle size does not significantly change the spread of the main laser beam.

The front face escape probability of PSL photons for various particle sizes can be seen in figure 4.16. Increasing the particle size leads to a decrease in the

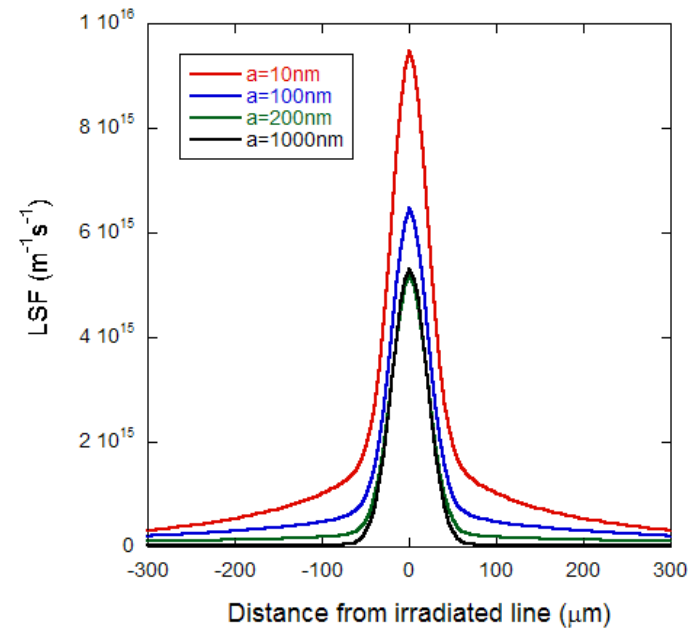

(a)

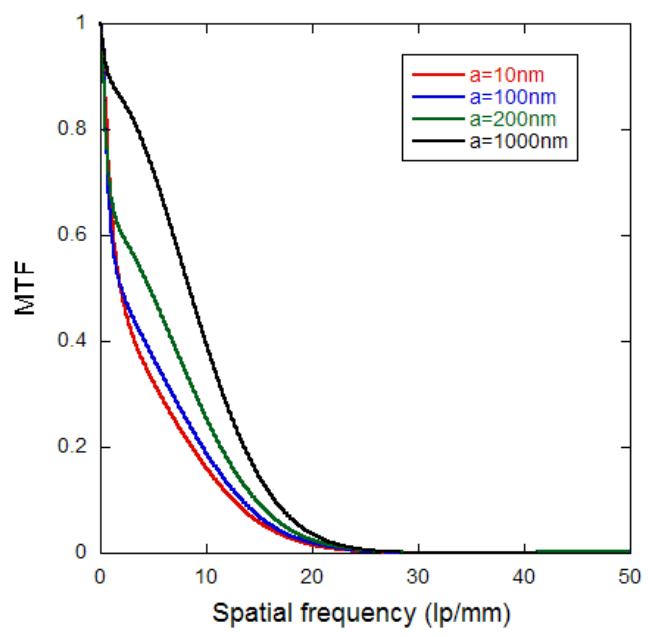

(b)

Figure 4.17: (a) LSF's and (b) MTF's for photons escaping from the front surface of the plate with various particle sizes. 


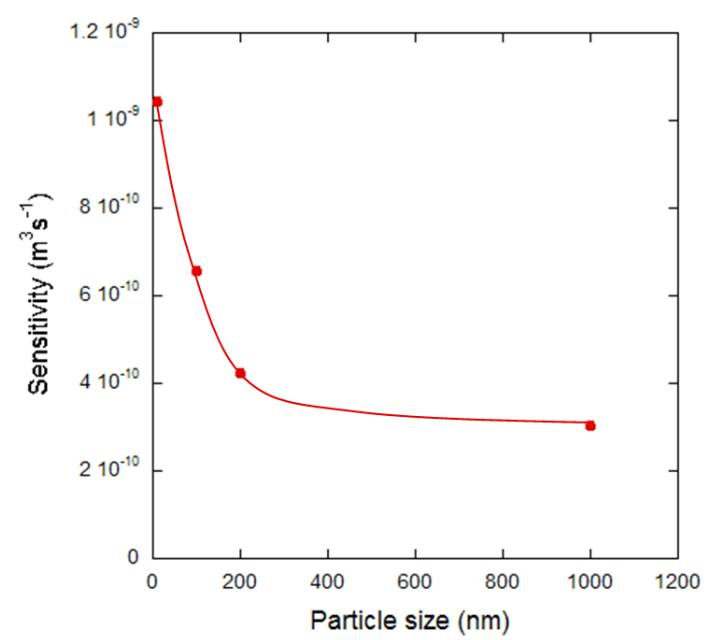

(a)

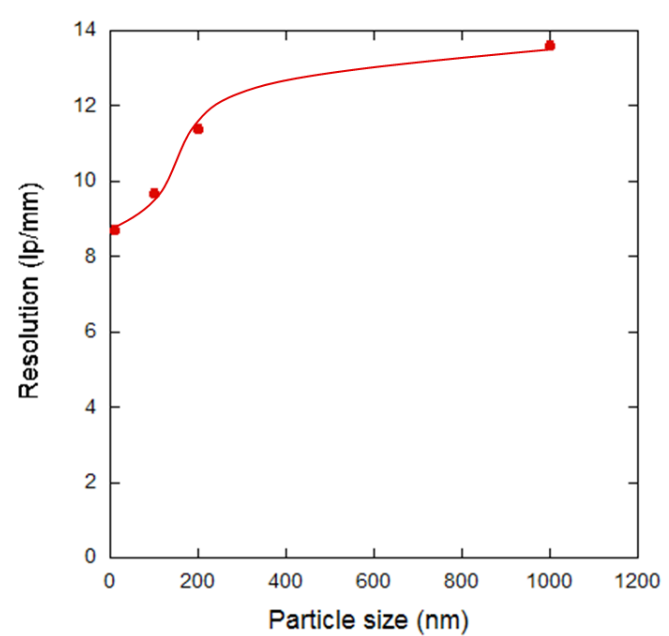

(b)

Figure 4.18: (a) Sensitivity and (b) Resolution plots for photons escaping from the front of the plate with various particles sizes. The lines are guides for the eye.

probability of escape.

Figure 4.17 shows the LSF and MTF curves for various particle sizes. Increasing the particle size increases the degree of forward scatter which results in both a decreasing LSF height, as photons have a shorter, more direct path to exit and an increasingly narrow tail as the lateral spread is reduced. The MTF reflects the LSF dependencies - a greater MTF width is achieved with larger particles due to the increased forwards scattering.

Sensitivity and resolution plots for the various particle sizes are presented in figure 4.18. The sensitivity decreases and resolution increases with increasing particle size.

\subsection{Effect of surface reflection}

In some commercial systems the imaging plate has additional layers which prevent reflection of the laser light back into the plate from the rear face. Simulations have been run for two different scattering lengths to investigate the effect including or not including reflection has on the resolution and sensitivity of the imaging plate. Two different scattering lengths were chosen to reflect 


\begin{tabular}{ccc}
\hline Parameter & Description & Value \\
\hline$\mu$ & Mean scattering length & $20,2000 \mu \mathrm{m}$ \\
$\gamma$ & Absorption coefficient & $0.001 \mu \mathrm{m}^{-1}$ \\
$g_{1}$ & Anisotropy coefficient for laser light & 0.5003 \\
$g_{2}$ & Anisotropy coefficient for PSL light & 0.7602 \\
$P$ & Laser power & $0.1 \mathrm{~mW}$ \\
$w$ & Laser variance & $20 \mu \mathrm{m}$ \\
$\eta$ & X-ray attenuation coefficient & $0.016 \mu \mathrm{m}^{-1}$ \\
\hline
\end{tabular}

Table 4.7: Parameters for simulations for the cases of reflection or no reflection from surfaces.

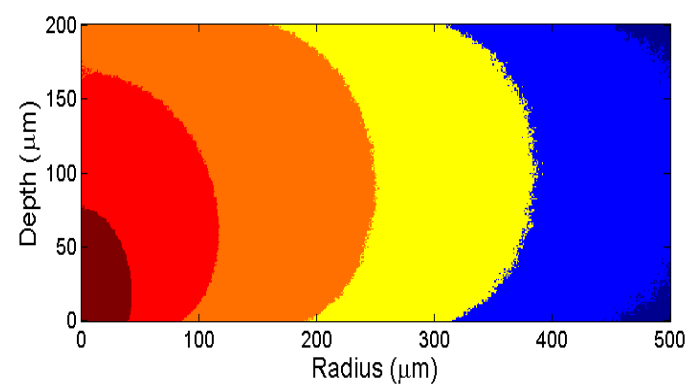

(a) No reflection. $\mu=20 \mu \mathrm{m}$

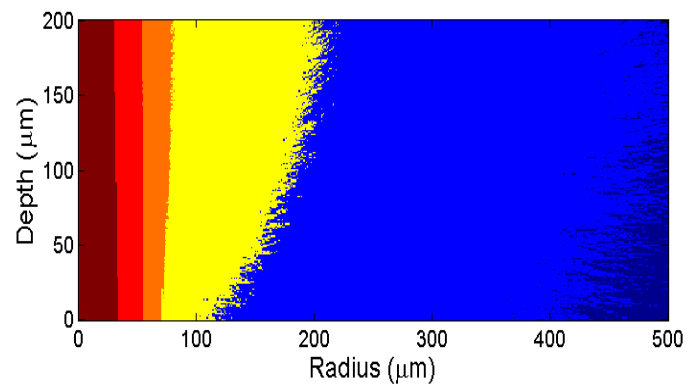

(c) No reflection. $\mu=2000 \mu \mathrm{m}$

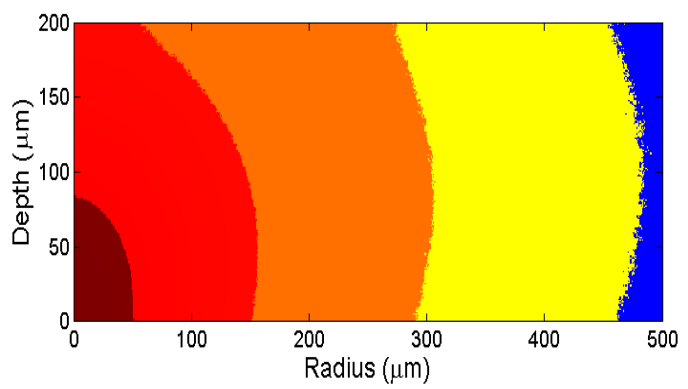

(b) Reflection. $\mu=20 \mu \mathrm{m}$

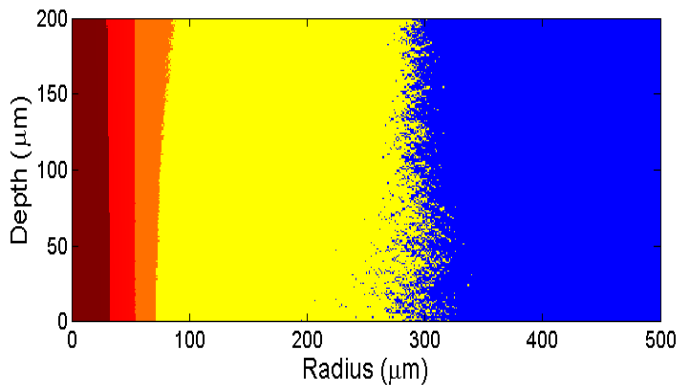

(d) Reflection. $\mu=2000 \mu \mathrm{m}$

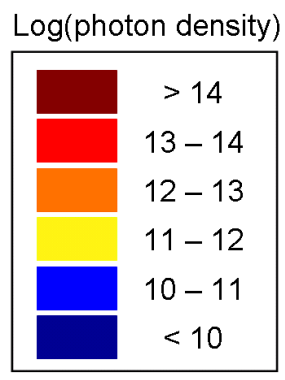

(e) key

Figure 4.19: (a-d) Intensity distribution profiles for $\mu=20 \mu \mathrm{m}$ and $\mu=2000$ $\mu \mathrm{m}$ including and not including reflection from surfaces as shown. (f) Logarithmic scale of the photon densities. 
the different forms the spread of laser photons can take. In these simulations the case of no reflection from any surface (as done by Fasbender et al [18]) is compared with the case of reflection from both surfaces. Parameters used in these simulations are presented in table 4.7 .

Intensity distributions for scattering lengths of $20 \mu \mathrm{m}$ and $2000 \mu \mathrm{m}$ for both reflection cases are shown in figure 4.19. When there is no reflection off any surface (figure 4.19 (a) and (c)) the spread of laser photons is significantly reduced compared to when reflection is included (figure 4.19 (b) and (d)) because the packets only travel once through the plate and their total path length is much shorter.

The probability of escape from the front surface for the cases of reflection and no reflection can be seen in figure 4.20. For a scattering length of $20 \mu \mathrm{m}$ the front face escape probability for photons generated near the front of the plate with no reflection from any face (red curve) increases as compared to reflection from both faces (blue curve) due to the shorter path length photons travel before escaping. The front face probability of escape for photons generated near the back of the plate decreases when reflection is not included, simply because of the greater path length required to exit from the front face. For a scattering length of $2000 \mu \mathrm{m}$ (green and black curves on figure 4.20) the front face escape

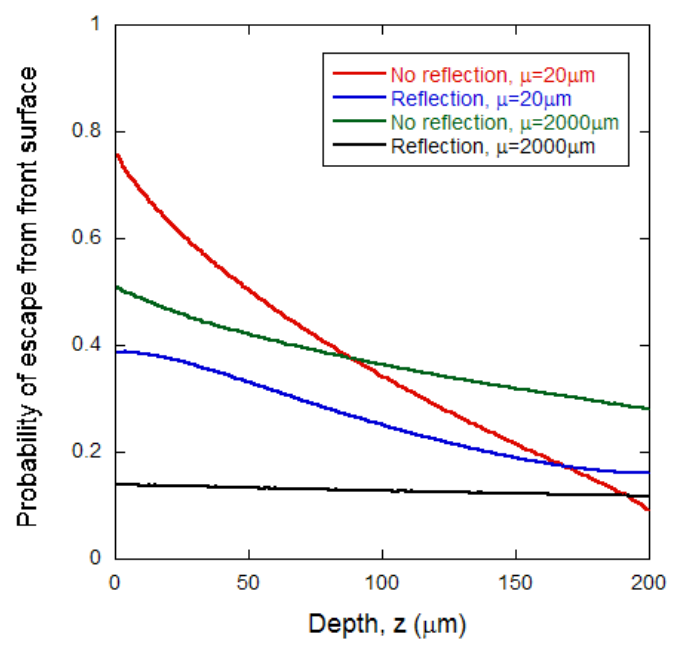

Figure 4.20: Probability of escape from the front surface of PSL photons generated at a depth in the plate for $\mu=20 \mu \mathrm{m}$ and $\mu=2000 \mu \mathrm{m}$ for the case of reflection or no reflection from surfaces. 
probability for the case of no reflection (green curve) increases as compared to the case of reflection from both faces (black curve) and is caused by the packets only travelling once through the plate.

The LSF's and MTF's for scattering lengths of $20 \mu \mathrm{m}$ and $2000 \mu \mathrm{m}$ for both reflection cases are shown in figure 4.21 . When reflection from any surface is not included (red and green curves) the amplitude of the LSF increases and the scattering tails are reduced for both scattering lengths when compared to reflection from both faces (blue and black curves). This is caused by a reduction in the spread of scattered laser photons. For both scattering lengths the width of the MTF increases for the case of no reflection as compared to including reflection, again due to the reduction in spread of the laser photons. The bimodal nature seen for $\mu=2000 \mu$ m with reflection off both faces (black curve on figure 4.21(b)) is reduced when reflection is not included (green curve).

These results show that when reflection from surfaces is not included the resolution and sensitivity are increased.

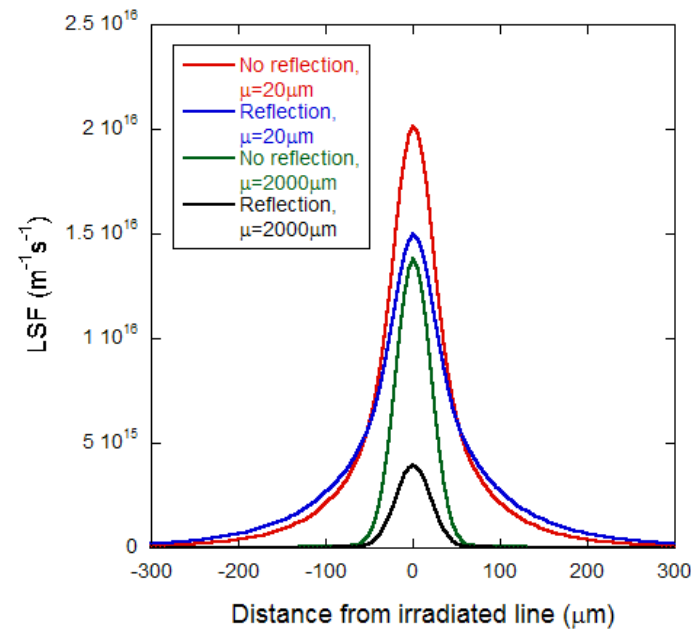

(a)

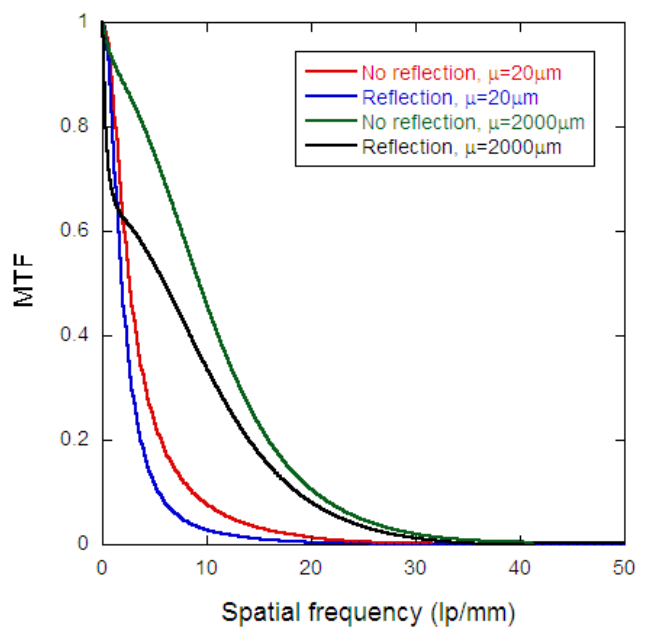

(b)

Figure 4.21: (a) LSF's and (b) MTF's for photons escaping from the front surface for $\mu=20 \mu \mathrm{m}$ and $\mu=2000 \mu \mathrm{m}$ including or not including reflection from surfaces. 


\subsection{Variations in laser power}

Varying the laser power changes the number of photons which are incident on the plate and hence the fraction of centres which are bleached during a scan. Strong bleaching is expected to adversely affect the resolution. In these simulations the laser power was varied from $0.1 \mathrm{~mW}$ to $10 \mathrm{~mW}$. The parameters are presented in table 4.8 .

Intensity distributions for the various laser powers are not shown as they are simply scale models of each other. The initial distribution with a laser power of $0.1 \mathrm{~mW}$ can be found in section 4.2 as figure 4.1 (c). Variations in laser power have no effect on the probability of escape of PSL photons and have not been included.

Figure 4.22 shows the LSF and MTF for the varying laser powers. The LSF curves are obtained by scanning the laser from positive to negative distances with the irradiated line sitting at a distance equal to 0 . Increasing the laser power increases the LSF amplitude as there are more photons in the plate to stimulate the line. At high laser powers the peak of the LSF curve reaches a maximum before the line due to the line of irradiated storage centres being partially bleached before the centre of the laser beam reaches it. This bleaching effect also causes the LSF curves for high laser powers to have an asymmetrical shape and broaden. By decreasing the laser power the LSF peak moves towards zero and the curves become symmetrical as less of the line is bleached

\begin{tabular}{ccc}
\hline Parameter & Description & Value \\
\hline$\mu$ & Mean scattering length & $200 \mu \mathrm{m}$ \\
$\gamma$ & Absorption coefficient & $0.001 \mu \mathrm{m}^{-1}$ \\
$g_{1}$ & Anisotropy coefficient for laser light & 0.5003 \\
$g_{2}$ & Anisotropy coefficient for PSL light & 0.7602 \\
$P$ & Laser power & $0.1-10 \mathrm{~mW}$ \\
$w$ & Laser variance & $20 \mu \mathrm{m}$ \\
$\eta$ & X-ray attenuation coefficient & $0.016 \mu \mathrm{m}^{-1}$ \\
\hline
\end{tabular}

Table 4.8: Parameters for simulations with varying laser powers. 


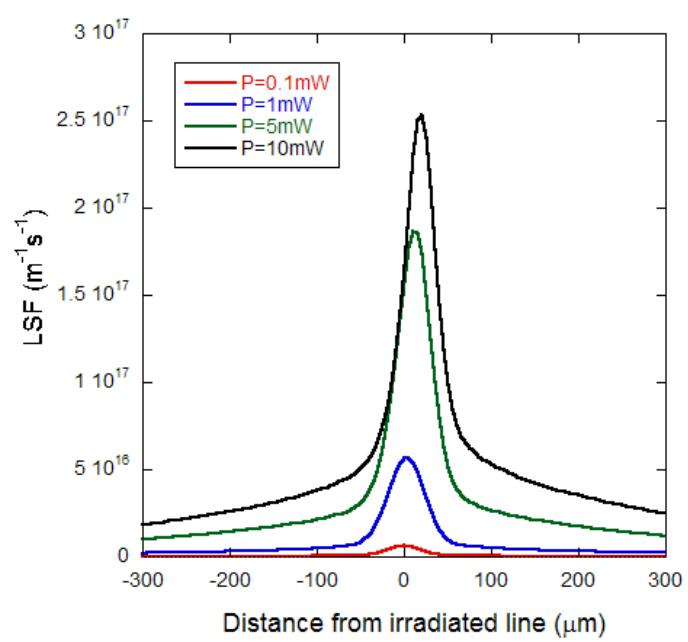

(a)

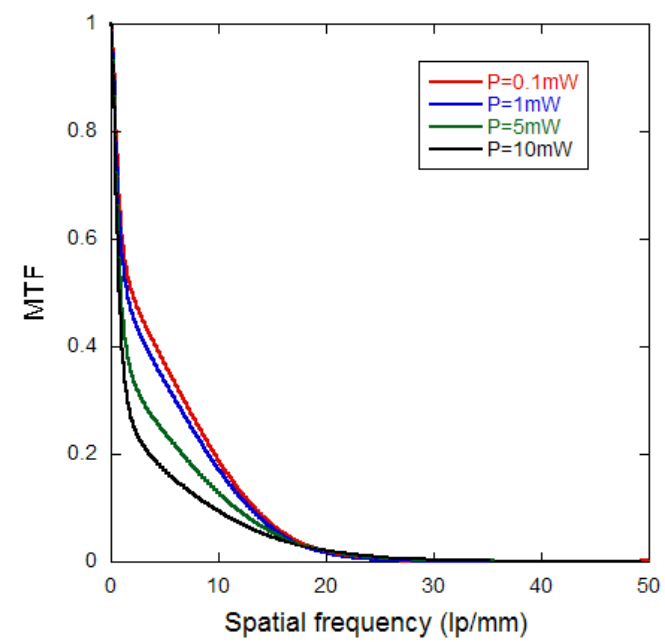

(b)

Figure 4.22: (a) LSF's, (b) MTF's for photons escaping from the front surface with varying laser powers as shown.

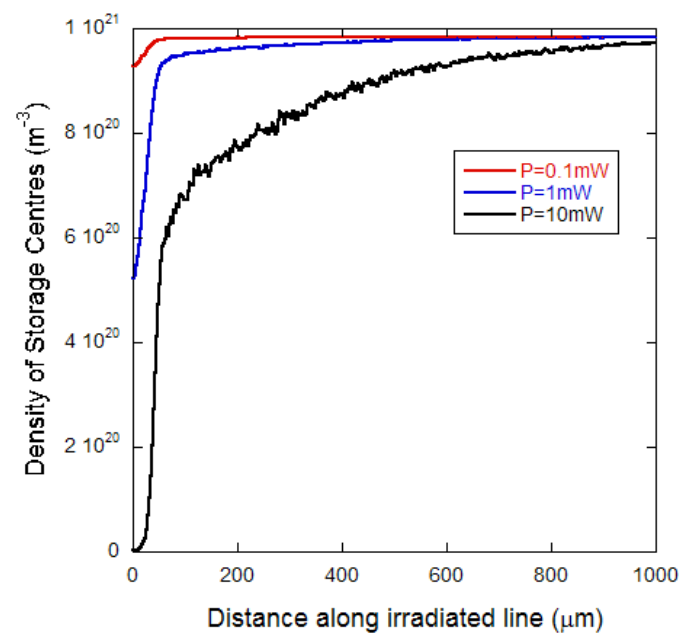

Figure 4.23: Variations in storage centre density with different laser powers.

with each step. At high and very low spatial frequencies the MTF is the same for each laser power. At intermediate spatial frequencies the MTF's diverge with the lowest laser power extending to higher values which is caused by the broadening of the LSF's.

In figure 4.23 the effect laser powers of $0.1,1$ and $10 \mathrm{~mW}$ has on the density of storage centres has been investigated. The graph shows the storage centre density plotted as a function of distance in the $y$ direction after the laser has scanned past the line. At high laser powers more storage centres are read-out and the width of the "hole" created by the laser increases. 


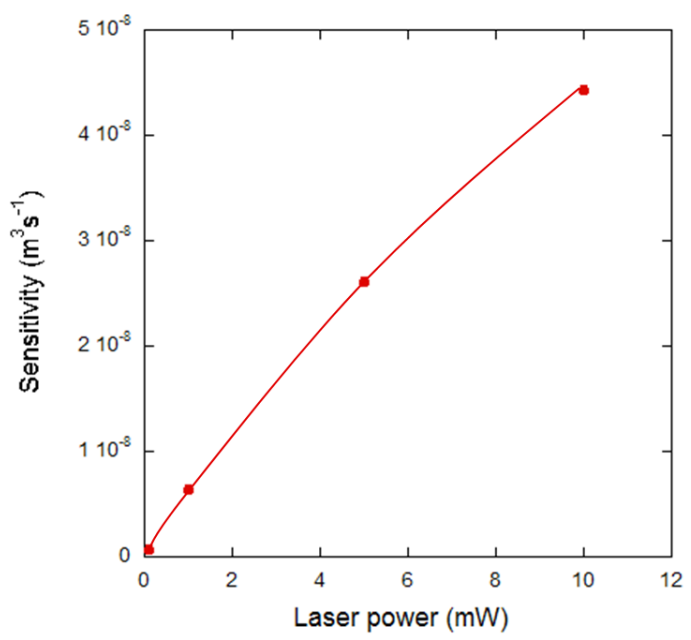

(a)

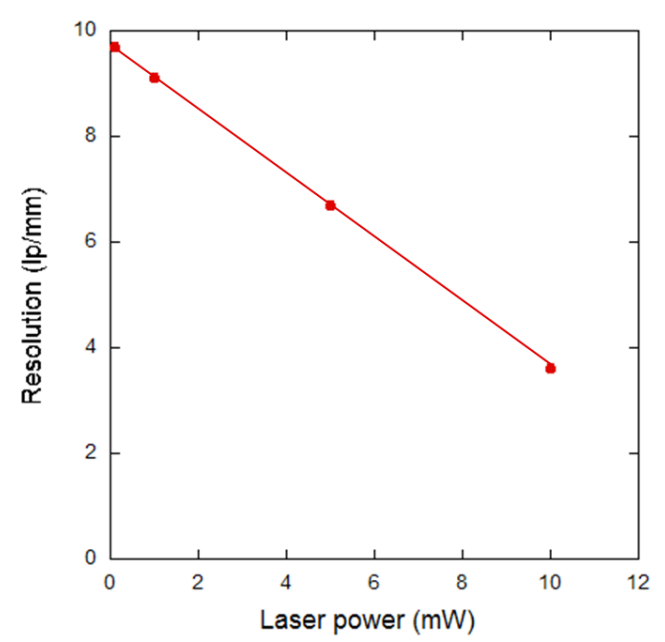

(b)

Figure 4.24: (a) Sensitivity and (b) Resolution plots for photons escaping from the front surface with varying laser powers. The lines are guides for the eye.

The resolution and sensitivity plots for the varying laser powers can be seen in figure 4.24. Increasing the laser power decreases the resolution as the LSF's are broadened and increases the sensitivity as more photons are incident on the plate. Note that all other calculations reported here have been for a power level of $0.1 \mathrm{~mW}$ which avoids power-induced broadening.

\subsection{Effect of laser beam variance}

The width of the focussed laser spot incident on the plate can be changed for example by using an aperture to narrow the laser beam. Usually the diameter of the laser is taken to be equal to $2 w$, where $w$ is the laser variance. Simulations

\begin{tabular}{ccc}
\hline Parameter & Description & Value \\
\hline$\mu$ & Mean scattering length & $200 \mu \mathrm{m}$ \\
$\gamma$ & Absorption coefficient & $0.001 \mu \mathrm{m}^{-1}$ \\
$g_{1}$ & Anisotropy coefficient for laser light & 0.5003 \\
$g_{2}$ & Anisotropy coefficient for PSL light & 0.7602 \\
$P$ & Laser power & $0.1 \mathrm{~mW}$ \\
$w$ & Laser variance & $5-20 \mu \mathrm{m}$ \\
$\eta$ & X-ray attenuation coefficient & $0.016 \mu \mathrm{m}^{-1}$ \\
\hline
\end{tabular}

Table 4.9: Parameters for simulations with varying laser variances. 


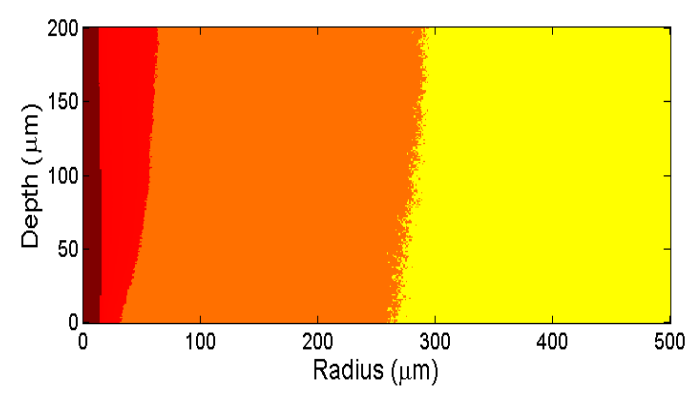

(a) $w=5 \mu \mathrm{m}$

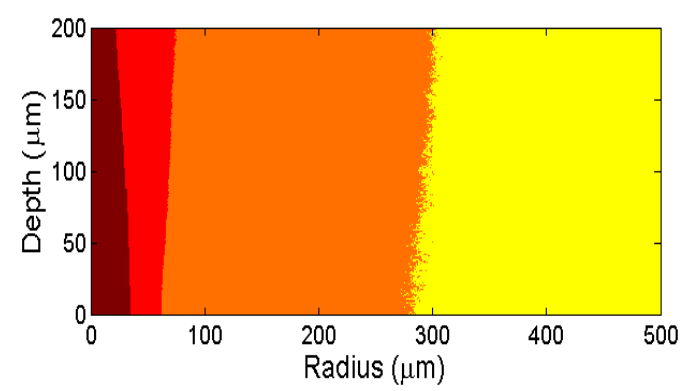

(c) $w=20 \mu \mathrm{m}$

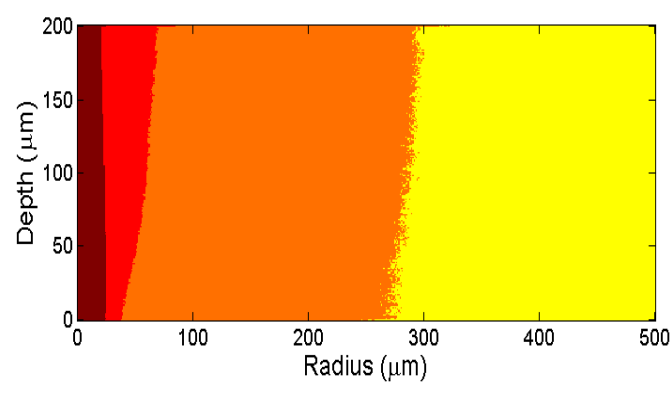

(b) $w=10 \mu \mathrm{m}$

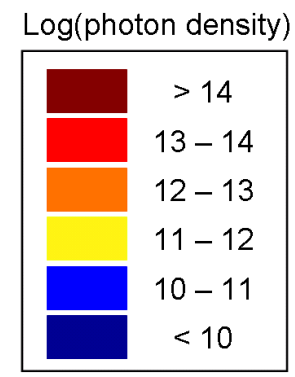

(d) key

Figure 4.25: $(\mathrm{a}-\mathrm{c})$ Intensity distribution profiles for varying the laser variance as shown. (d) Logarithmic scale of the photon densities.

were run with the parameters given in table 4.9.

Decreasing the laser variance decreases the width of the main laser beam as can be seen in the intensity distributions in figure 4.25 , but the spread far away from the main beam due to scattering of laser photons does not change significantly for the different variances.

The probability of escape of PSL photons does not change with varying laser variance and has not been shown.

LSF's and MTF's for the different laser variances can be seen in figure 4.26. Decreasing the variance decreases the width of the LSF, reflecting the narrowing of the laser beam, and increases the height due to the same laser power being compressed into a smaller area. The scattering tails on the LSF are not changed with varying laser variance which reflects the small change seen in the intensity distributions. The resolution at a MTF equal to 0.2 increases with decreasing variance as expected. At low frequencies the MTF curves are the same due to the scattering tails not changing and at high frequencies the different widths of the laser beams are reflected directly in the different MTF's. 


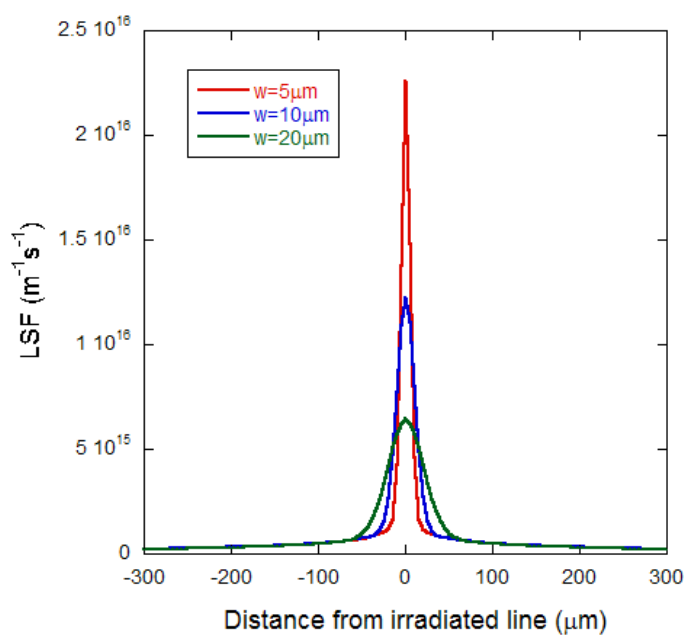

(a)

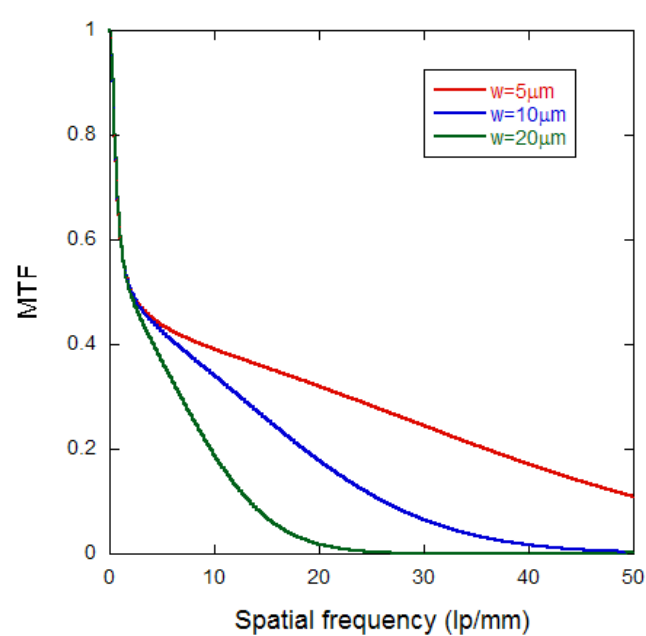

(b)

Figure 4.26: (a) LSF's and (b) MTF's for photons escaping from the front surface with various laser variances.

For the lowest laser variance the MTF extends to much higher spatial frequencies and the plate is able to distinguish finer detail in images.

Figure 4.27 shows the resolution and sensitivity plots for varying the laser variance. Both the resolution and sensitivity increase with decreasing laser variance, with the resolution increasing significantly for small laser variances. The sensitivity was not expected to change with variations in laser variance as the power is held fixed, and only a slight deviation is seen.

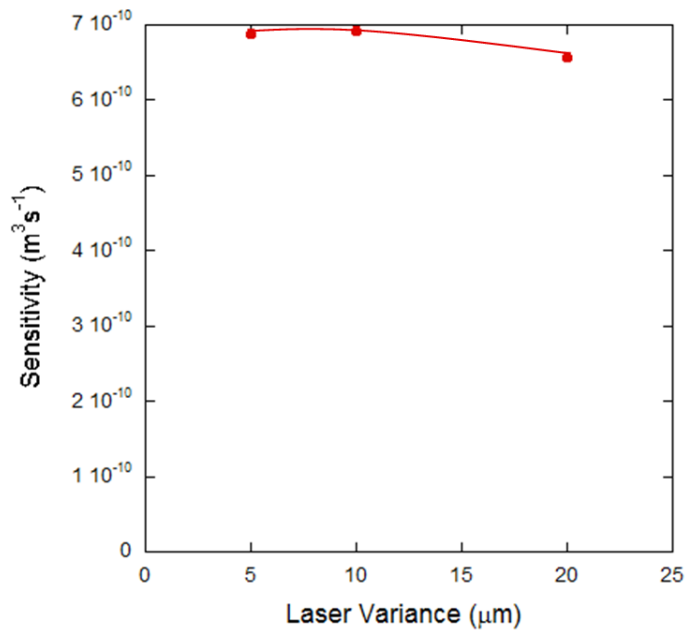

(a)

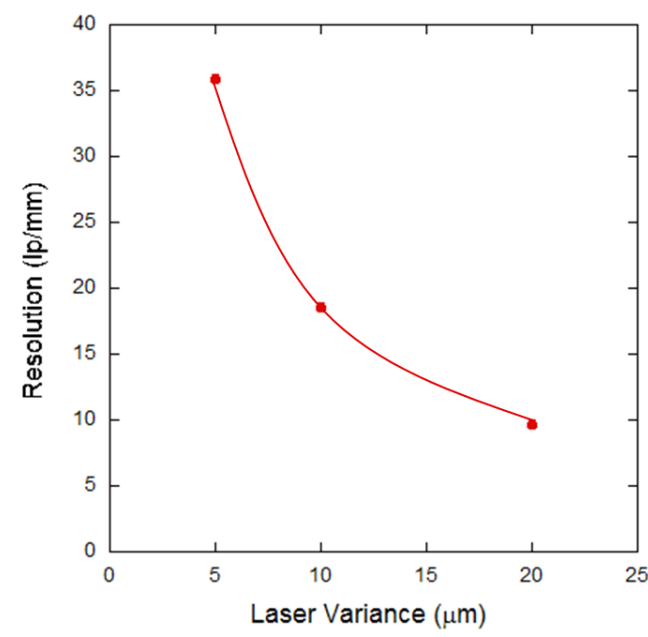

(b)

Figure 4.27: (a) Sensitivity and (b) Resolution plots for photons escaping from the front surface with various laser variances. The lines are guides for the eye. 


\subsection{Dependence on x-ray attenuation coefficient}

The x-ray attenuation coefficient determines the distribution of storage centres through the depth of the plate, with the concentration of centres decreasing exponentially with increasing depth. Equation 3.25 in section 3.10 describes the relationship between $x$-ray attenuation coefficient and the number of storage centres. The attenuation coefficient is strongly dependent on the composition of the plate and the energy of the incident x-rays. Generally, a lower x-ray energy corresponds to a higher attenuation coefficient and the x-rays do not penetrate as far into the plate. Simulations were run to investigate the effect of varying the x-ray attenuation coefficient for both strong and weak scattering. Parameters for these simulations are presented in table 4.10.

The attenuation coefficients chosen are appropriate to a basic fluorozirconate glass ceramic. Table 4.11 shows how these attenuation coefficients vary with

\begin{tabular}{ccc}
\hline Parameter & Description & Value \\
\hline$\mu$ & Mean scattering length & $20,2000 \mu \mathrm{m}$ \\
$\gamma$ & Absorption coefficient & $0.001 \mu \mathrm{m}^{-1}$ \\
$g_{1}$ & Anisotropy coefficient for laser light & 0.5003 \\
$g_{2}$ & Anisotropy coefficient for PSL light & 0.7602 \\
$P$ & Laser power & $0.1 \mathrm{~mW}$ \\
$w$ & Laser variance & $20 \mu \mathrm{m}$ \\
$\eta$ & X-ray attenuation coefficient & $0.00013-0.016 \mu \mathrm{m}^{-1}$ \\
\hline
\end{tabular}

Table 4.10: Parameters for simulations with varying $\mathrm{x}$-ray attenuation coefficients.

\begin{tabular}{ccc}
\hline Energy $(\mathrm{keV})$ & $\eta\left(\mu \mathrm{m}^{-1}\right)$ & Half length $(\mu \mathrm{m})$ \\
\hline 15 & 0.0093 & 75 \\
20 & 0.016 & 43 \\
40 & 0.0038 & 180 \\
60 & 0.0013 & 530 \\
100 & 0.00034 & 2000 \\
150 & 0.00013 & 5300 \\
\hline
\end{tabular}

Table 4.11: Relationship between x-ray energy, attenuation coefficient and half length for a fluorozirconate glass. The half length is the depth at which the $\mathrm{x}$-ray intensity reduces to half its initial value. 


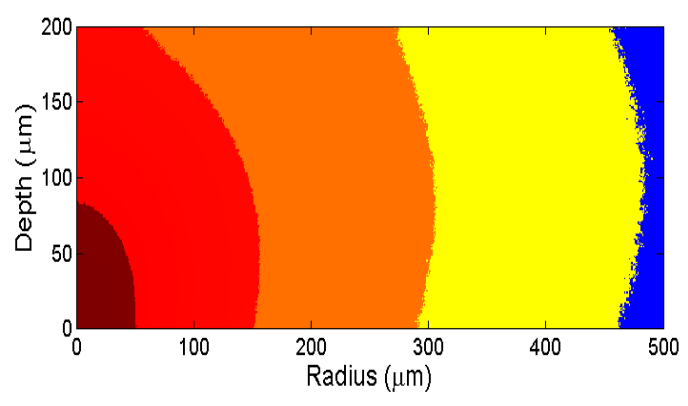

(a)

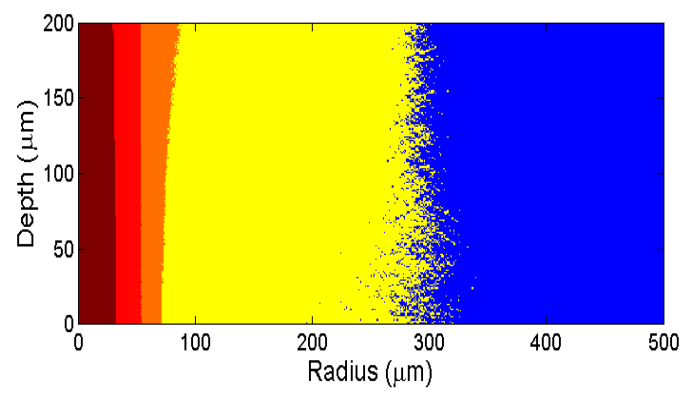

(b)

Figure 4.28: Intensity distribution profiles for scattering lengths of (a) $20 \mu \mathrm{m}$ and (b) $2000 \mu \mathrm{m}$. The colour key is the same as for previous intensity distributions.

x-ray energy. The half length decreases at first due to an edge in the x-ray attenuation characteristic caused by core level transitions in the atoms which make up the fluorozirconate glass. From this point onwards the variation will be stated in terms of x-ray energy and not attenuation coefficient.

For the purposes of discussion, stimulating light intensity distributions from section 4.2 are replicated in figure 4.28 for scattering lengths of $20 \mu \mathrm{m}$ and 2000 $\mu \mathrm{m}$. The intensity distributions and probability of escape of PSL photons do not change with variations in x-ray energy.

The LSF and MTF for simulations for varying x-ray energies with a scattering length of $20 \mu \mathrm{m}$ can be seen in figure 4.29. The LSF's had to be scaled by the attenuation coefficient $\eta$, as the simulations did not take into account the dependence of the initial density of storage centres on the x-ray energy. The corrected dependence of the distribution of storage centres through the plate on x-ray energy is given in equation 4.1.

$$
n_{\mathrm{sc}}^{\prime}=\eta n_{\mathrm{sc}} \exp (-\eta z)
$$

The principal effect of varying the x-ray energy is that for small $(15-20 \mathrm{keV})$ energies a high density of storage centres is concentrated near the surface, whilst for high energies $(100-150 \mathrm{keV})$ less energy is absorbed and so the storage centres have a lower density but which is uniformly distributed through 


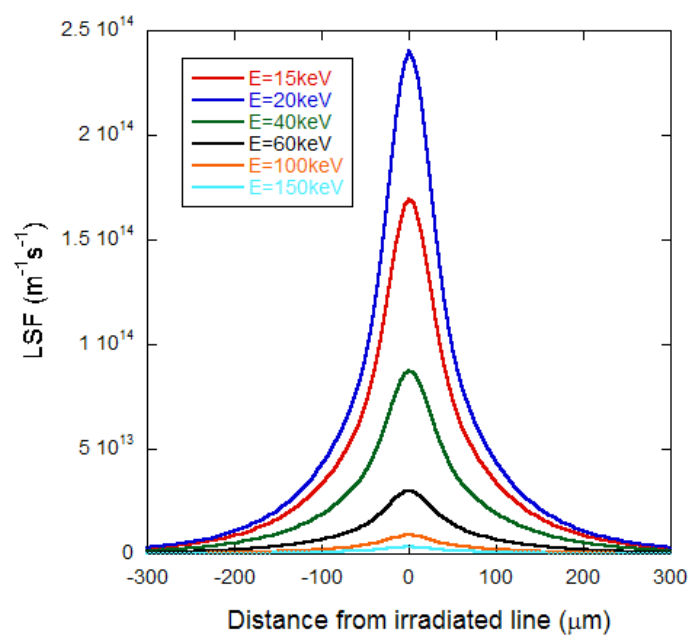

(a)

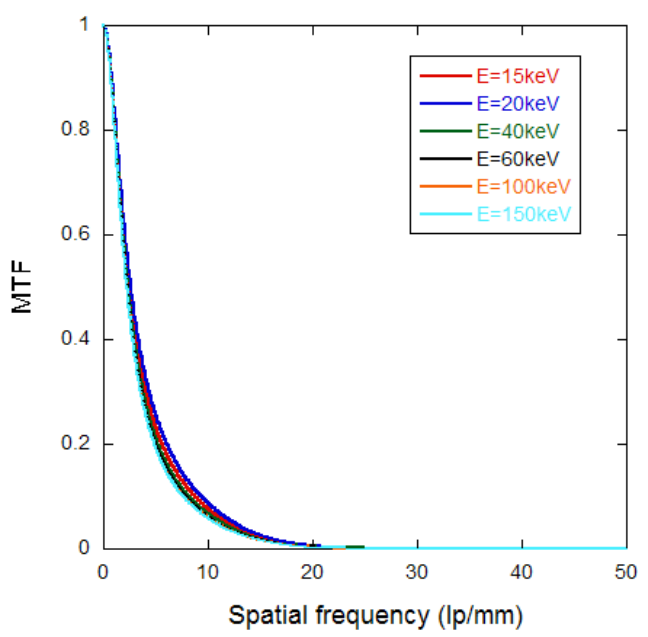

(b)

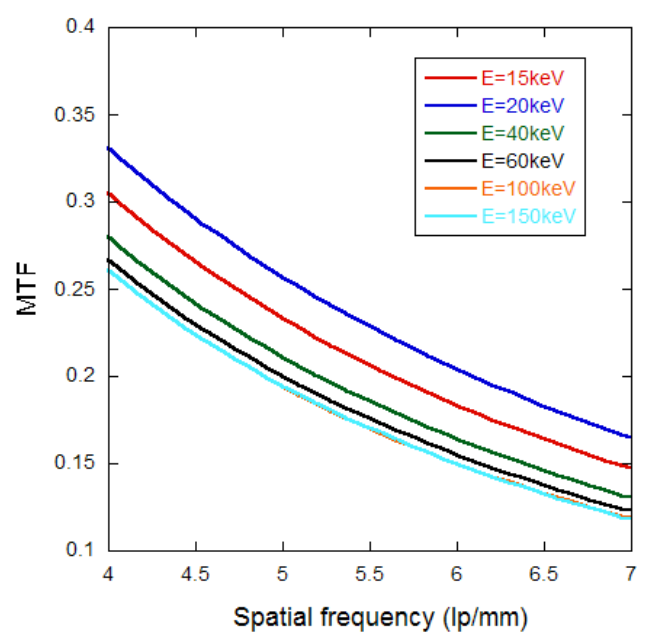

(c)

Figure 4.29: (a)LSF's and (b) MTF's for photons escaping from the front surface with various x-ray energies and a scattering length of $20 \mu \mathrm{m}$. (c) Expanded MTF for spatial frequencies between 4 and $7 \mathrm{lp} / \mathrm{mm}$.

the thickness.

Increasing the x-ray energy from 15 to $20 \mathrm{keV}$ causes the LSF amplitude to increase as more storage centres are generated in the plate because of the higher attenuation coefficient for $20 \mathrm{keV}$. Increasing the x-ray energy further, the height of the LSF decreases as more x-rays pass straight through the plate and fewer storage centres are generated. At high x-ray energies there is a slight broadening of the LSF which is caused by a more uniform distribution of centres through the plate. The combination of a shallow and dense concentration of storage centres for low x-ray energies and the density of stimulating photons 


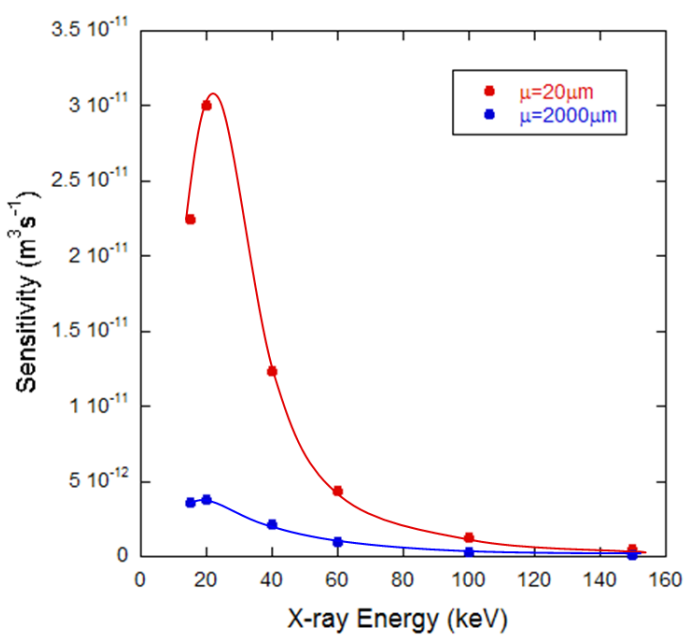

(a)

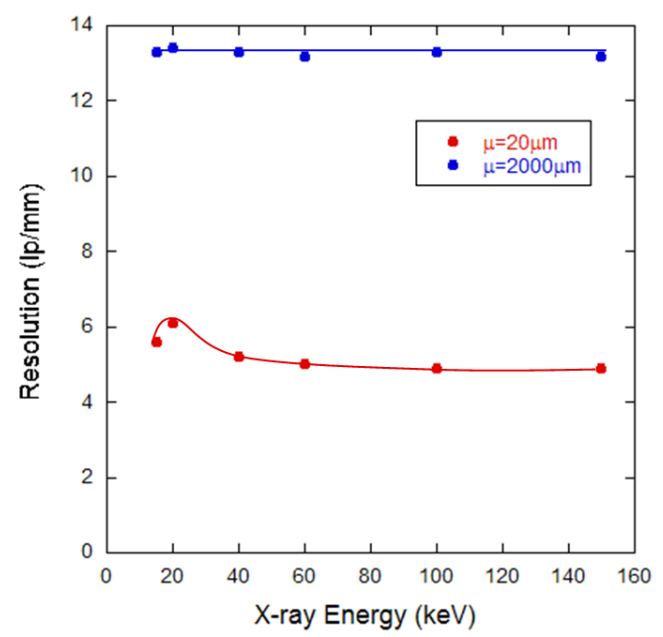

(b)

Figure 4.30: (a) Sensitivity and (b) Resolution plots for photons escaping from the front surface with various $x$-ray energies and scattering lengths of $20 \mu \mathrm{m}$ (red dots) and $2000 \mu \mathrm{m}$ (blue dots). The lines are guides for the eye.

near the surface leads to a sharper LSF than for high energies. The sharpening of the LSF is reflected as a broadening of the MTF. To better examine the trends, figure 4.29(c) shows the MTF expanded for spatial frequencies of 4 to 7 $\mathrm{lp} / \mathrm{mm}$. Increasing the $\mathrm{x}$-ray energy from 15 to $20 \mathrm{keV}$ increases the width of the MTF in this range, and any further increase in energy decreases the width of the MTF.

Resolution and sensitivity plots for the varying $\mathrm{x}$-ray energies with a scattering length of $20 \mu \mathrm{m}$ are shown as the red dots in figure 4.30. The sensitivity increases at low energies due to the largest attenuation coefficient occurring at $20 \mathrm{keV}$, and then decreases at higher energies. For high energies, very little energy is deposited in the plate and so the density of storage centres is small. The resolution also increases for an energy of $20 \mathrm{keV}$ which is caused by the broadening of the LSF's for all other energies.

Simulations of the LSF and MTF for various x-ray energies with a scattering length of $2000 \mu \mathrm{m}$ are shown in figure 4.31. The trends for both the LSF and MTF are similar to those shown for $\mu=20 \mu \mathrm{m}$, but there is no significant broadening of the LSF at high energies as was seen previously, which results in the MTF's being the same for each energy. 


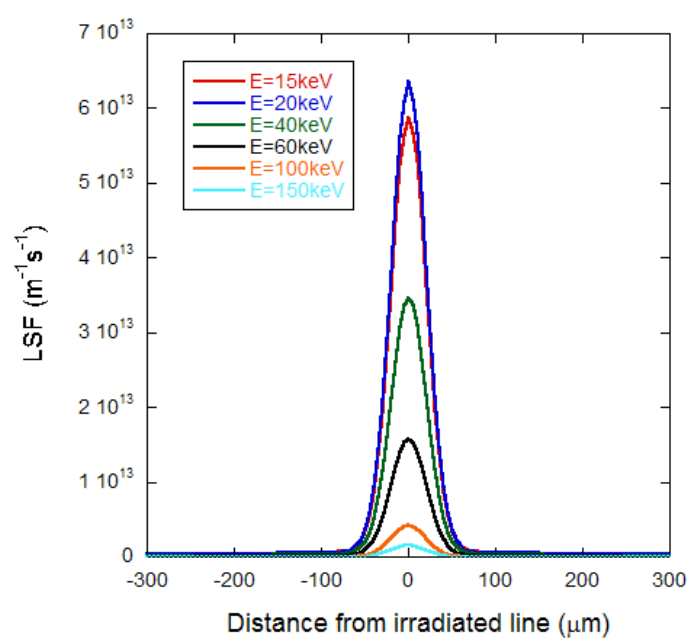

(a)

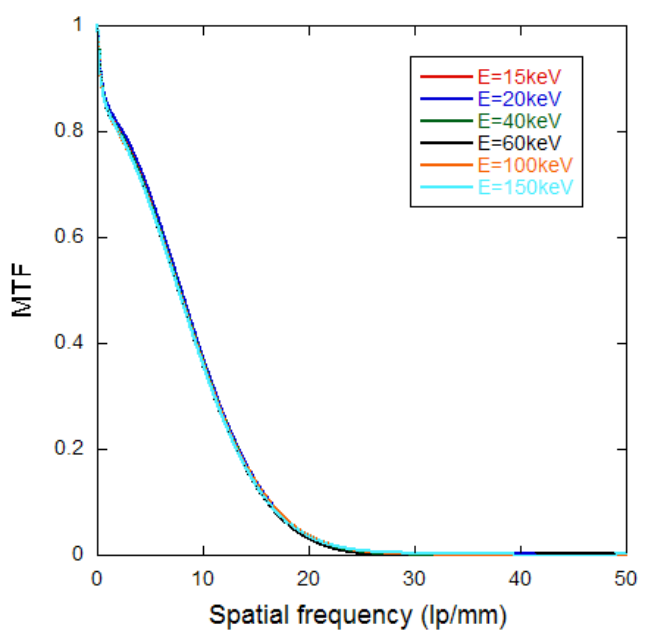

(b)

Figure 4.31: (a) LSF's and (b) MTF's for photons escaping from the front surface with various $x$-ray energies and a scattering length of $2000 \mu \mathrm{m}$.

Again the resolution and sensitivity for a scattering length of $2000 \mu \mathrm{m}$ (blue dots in figure 4.30) show similar trends but the different density distribution for the stimulating light (figure 4.28(b)) leads to a lower sensitivity but higher resolution. 


\section{Chapter 5}

\section{Experimental Techniques}

\subsection{Glass ceramic sample preparation}

A typical glass ceramic sample is composed of $52 \mathrm{ZrF}_{4}, 10.3 \mathrm{BaF}_{2}, 10 \mathrm{BaCl}_{2}, 20$ $\mathrm{NaCl}, 4 \mathrm{LaF}_{3}, 3.5 \mathrm{AlF}_{3}$ and $0.2 \mathrm{EuF}_{2}$ in mole percent.

The dry materials are first weighed and mixed into a pre-cleaned dry Pt-Rh lidded crucible in an inert gas glove box of low water vapour and oxygen content. The crucible is then transferred to a horizontal tube furnace at a temperature of $\sim 750^{\circ} \mathrm{C}$ and then held at this temperature until all components have dissolved into the melt, approximately 90 minutes. When there are no visual signs of undissolved material in the melt, it is poured onto a pair of pre-heated, temperature-controlled, copper quenching plates held at a temperature of 190 ${ }^{\circ} \mathrm{C}$. The resultant glass is sandwiched between the plates and held at $\sim 190{ }^{\circ} \mathrm{C}$ for approximately two hours before being slow cooled to room temperature at $20^{\circ} \mathrm{C} / \mathrm{hr}$. The glass is then lapped and oil polished before being annealed.

The physical appearance of the as-made glass was clear with a small quantity of sub-millimetre sized bubbles. Differential scanning calorimetry (DSC) measurements using a Shimadzu DSC-600 of this particular glass ceramic showed a glass transition onset at $205^{\circ} \mathrm{C}$ and a crystallisation peak at $243{ }^{\circ} \mathrm{C}$ (figure 5.1); this data was used to guide the choice of quench block temperature.

The samples are then annealed in air by ramping up the temperature of the 


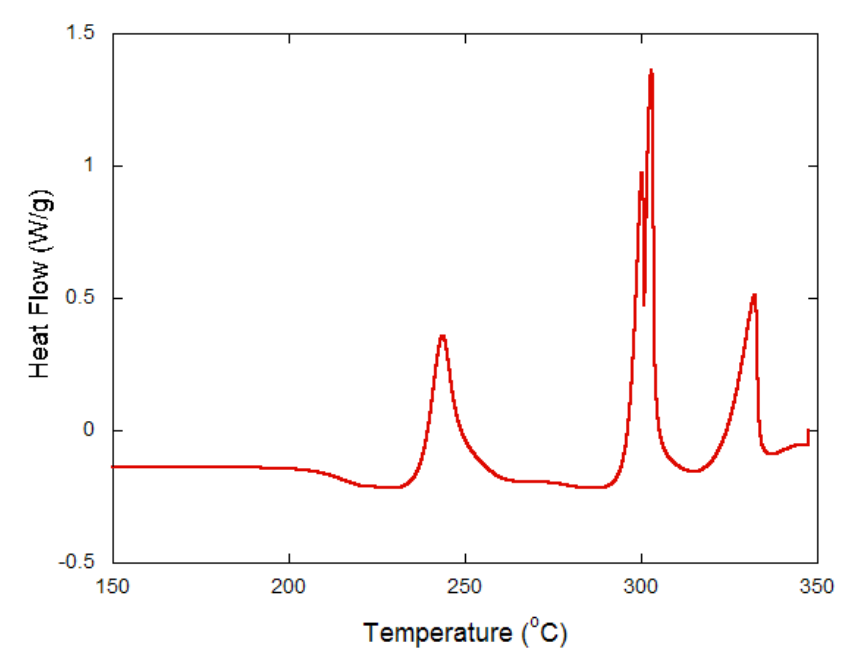

Figure 5.1: DSC measurements of a typical glass ceramic sample.

quenching block to above the crystallisation peak $\left(\sim 270^{\circ} \mathrm{C}\right)$ and holding at this temperature for around 30 minutes. The appearance of the glasses after annealing is typically near-transparent, yellow in transmitted light and blue in scattered light.

\subsection{Absorption and scattering}

A Shimadzu UV-Vis 2100 spectrophotometer was used to determine the extinction coefficient of various materials. Extinction includes attenuation of the beam by both absorption and scattering processes. The spectrophotometer uses a dual beam system where the incident light source is split into a sample and reference beam to measure the differences in light intensity. An optical schematic is shown in figure 5.2.

Light is emitted from either the deuterium or halogen lamp and is reflected by mirrors onto the monochromator. The light source is switched automatically at $\lambda \sim 370 \mathrm{~nm}$ depending on the wavelength of light which is required and the positions of the light sources are adjusted to obtain the maximum intensity. A monochromator is a device which selects a narrow band of wavelengths of light from a much wider band. The design of the monochromator in the UV- 


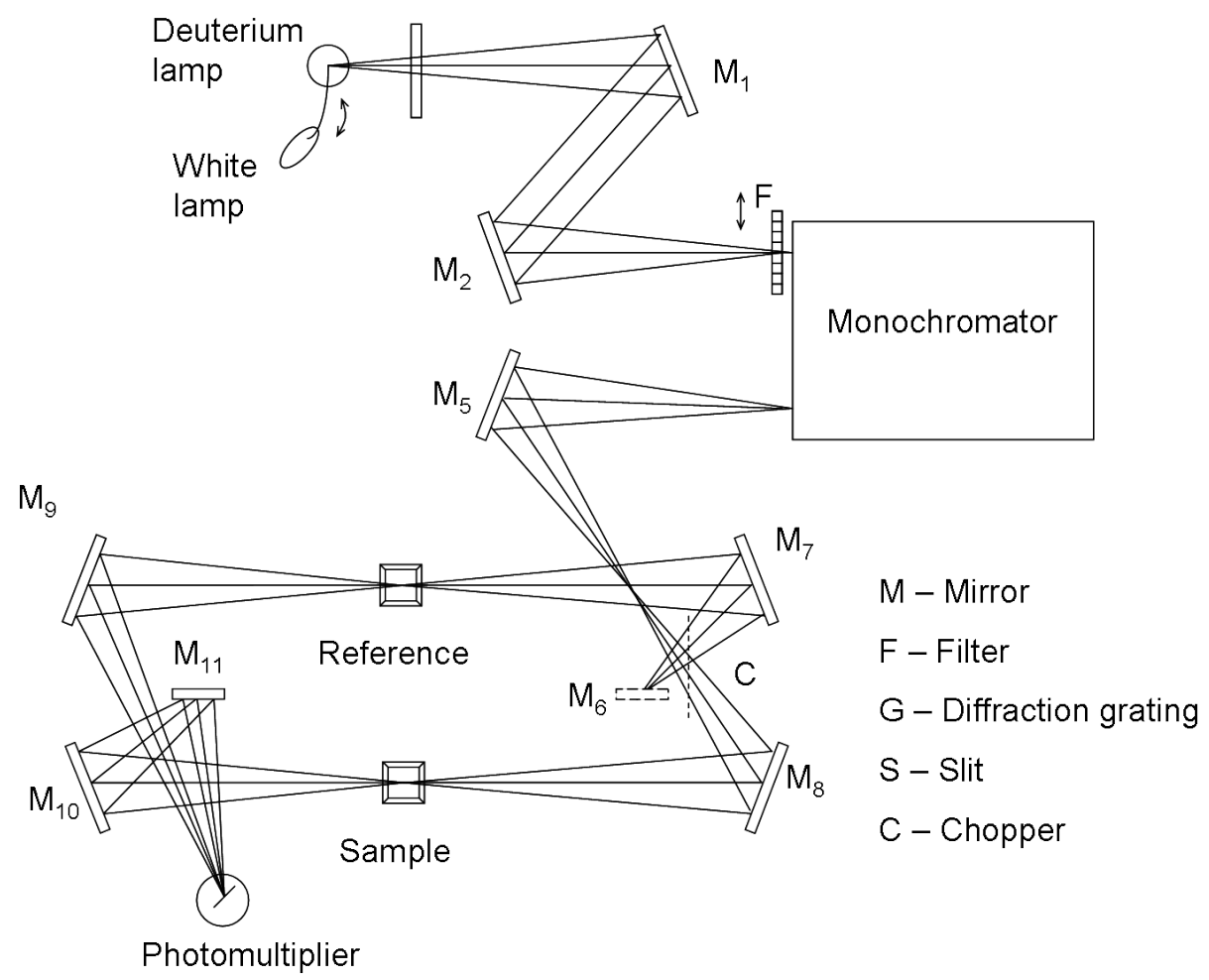

Figure 5.2: Optical schematic of Shimadzu UV-Vis 2100 spectrophotometer.

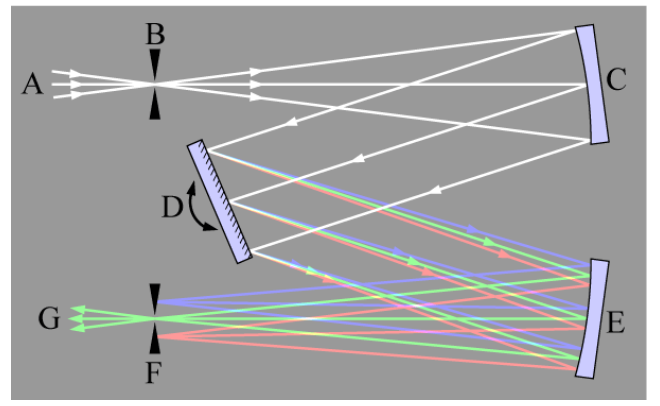

Figure 5.3: Czerny-Turner monochromator design. [27]

2100 is a Czerny-Turner monochromator (figure 5.3) which uses a diffraction grating to separate the wavelengths.

The broad spectrum of light from the source (A) passes through the entrance slit (B) and is incident on a curved mirror (C). The slit is placed at the focus point of the curved mirror so that the light reflected from the mirror is focussed at infinity and the light is collimated. The collimated light is then diffracted by the diffraction grating (D) and collected by another curved mirror (E) which refocusses the dispersed light onto the exit slit. Rotating the grating causes different wavelengths to exit through the slit. The slit width determines both 
the throughput of the spectrophotometer and the resolution. Both the entrance and exit slit are adjusted together to give an instrumental resolution of $0.1 \mathrm{~nm}$ to $5 \mathrm{~nm}$.

To measure extinction coefficients of a material the spectrophotometer is used in absorbance mode where the absorbance of light by the sample is measured as a function of the wavelength of light.

When light is passed through a sample the light intensity decreases due to absorption and scattering within the material, in accordance with equation 5.1 where $I$ is the intensity of the light at wavelength $\lambda$ which has passed through a sample, $I_{0}$ is the incident light intensity, $d$ is the thickness of the sample and $\alpha_{\text {ext }}$ is the extinction coefficient. The extinction coefficient includes both the coefficients for absorbed $\left(\alpha_{\mathrm{a}}\right)$ and scattered light $\left(\alpha_{\mathrm{s}}\right)$.

$$
I=I_{0} \exp \left(-\alpha_{\text {ext }} d\right)
$$

"Absorbance" refers to the mathematical quantity $A b s(\lambda)=-\log _{10}\left(I / I_{0}\right)$. Absorbance only calculates the ratio of transmitted light over incident light and does not distinguish the mechanism by which the light intensity decreases.

The absorption coefficient and scattering length of a glass ceramic sample can be separated as follows.

Before annealing measured "absorbance" is due to true absorption by the material and interfacial reflection. Equation 5.2 shows how the absorption coefficient can be determined from the measured absorbance where $R$ is the reflection coefficient.

$$
\alpha_{\mathrm{a}}=\frac{A b s(\lambda)+\log _{10}(1-R)^{2}}{d \log _{10}(e)}
$$

After the glass has been annealed it is assumed that any additional contribution is due to scattering by the crystallites. The scattering length $(\mu)$ is deter- 
mined by the following process.

Absorbance before annealing : $A b s_{\text {before }}(\lambda)=\alpha_{\mathrm{a}} d \log _{10}(e)+\log _{10}(1-R)^{2}$

Absorbance after annealing : $A b s_{\text {after }}(\lambda)=\left(\alpha_{\mathrm{a}}+\alpha_{\mathrm{s}}\right) d \log _{10}(e)+\log _{10}(1-R)^{2}$

$$
\begin{gathered}
A b s_{\text {after }}(\lambda)-A b s_{\text {before }}(\lambda)=\alpha_{\mathrm{s}} d \log _{10}(e) \\
\mu=\frac{1}{\alpha_{\mathrm{s}}}=\frac{d \log _{10}(e)}{A b s_{\text {after }}(\lambda)-A b s_{\text {before }}(\lambda)}
\end{gathered}
$$

\subsection{X-ray generator}

The x-ray generator used to create images of the objects was a Hewlett-Packard 43855A Faxitron. The Faxitron was designed to give high resolution images of any type of small to medium sized objects and is based on an x-ray tube with a very small focal spot $(50 \mu \mathrm{m})$.

Electrons are accelerated towards a tungsten anode of an angle of $20^{\circ}$ to the normal (figure 5.4). The $\mathrm{x}$-ray tube is capable of a $0.3 \mathrm{~mA}$ maximum continuous current and adjustable $10-55 \mathrm{kV}$ output voltage. To achieve high voltages the Faxitron must first be "warmed up" by running it at $0.3 \mathrm{~mA}$ and $30 \mathrm{kV}$ for around thirty minutes. The generated x-rays pass through a $38.1 \mathrm{~mm}$ diameter, $0.64 \mathrm{~mm}$ thick beryllium window and are incident on the object. The initial spread of the beam is $30^{\circ}$.

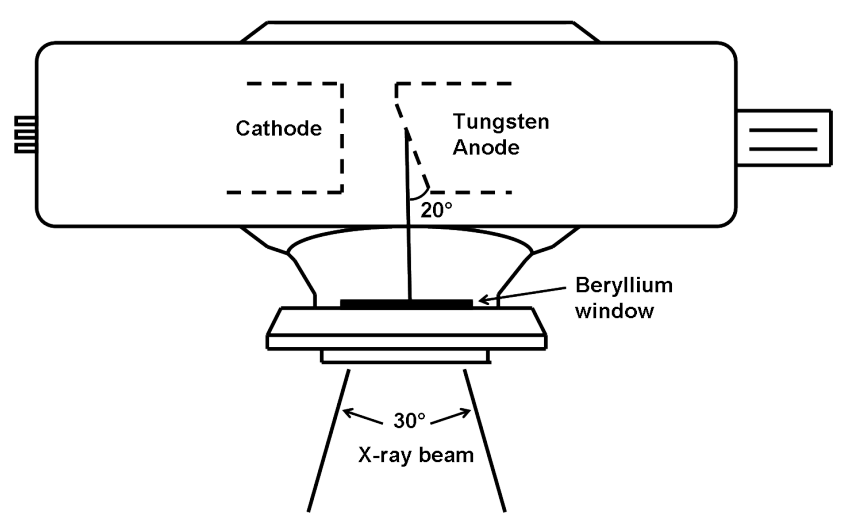

Figure 5.4: Diagram of Faxitron x-ray tube. 
The Faxitron has two shelf positions allowing the source to object distance to be $460 \mathrm{~mm}$ in the top position and $560 \mathrm{~mm}$ in the lowest position. This allows for beam diameters of $246 \mathrm{~mm}$ and $300 \mathrm{~mm}$ respectively. If a higher dose is required the distance between source and object can be decreased with the use of a small scissor lift.

\subsection{MTF measurements}

In commercial x-ray storage phosphor imaging systems, the image is read-out by raster scanning a focussed laser beam across the plate. The modulation transfer function can be determined in a similar way, by recovering the image of a test grid pattern or sharp edge object as a focussed laser beam is scanned across the plate.

Figure 5.5 shows a schematic of the experimental image read-out system and figure 5.6 is a photograph of the set up. In this case a $0.9 \mathrm{~mW} \mathrm{He}-\mathrm{Ne}$ laser is fixed and the sample is moved through the laser by the use of a translation stage. A beam expander is used to increase the width of the Gaussian profile laser beam to permit sharper focussing of the beam onto the imaging plate. Equation 5.4 [28] gives the relationship between the initial beam diameter $\left(w_{0}\right)$ and the beam diameter $(w)$ after focussing with a lens of focal length $f$.

$$
w=\frac{f \lambda}{\pi w_{0}}
$$

An aperture was initially used to limit the spread of the laser to the size of the focussing lens. However, in practice it was found that decreasing the size of the aperture further decreased the focussed laser spot size.

The laser is focussed onto the sample with a $2 \mathrm{~mm}$ focal length lens taken from a Fujitsu CDA48FA CR-ROM drive. The resulting PSL light is deflected by a partially reflecting gold mirror and then focussed onto a Brookhaven BI-DS photomultiplier tube using a $30 \mathrm{~mm}$ focal length lens. The partially reflecting 


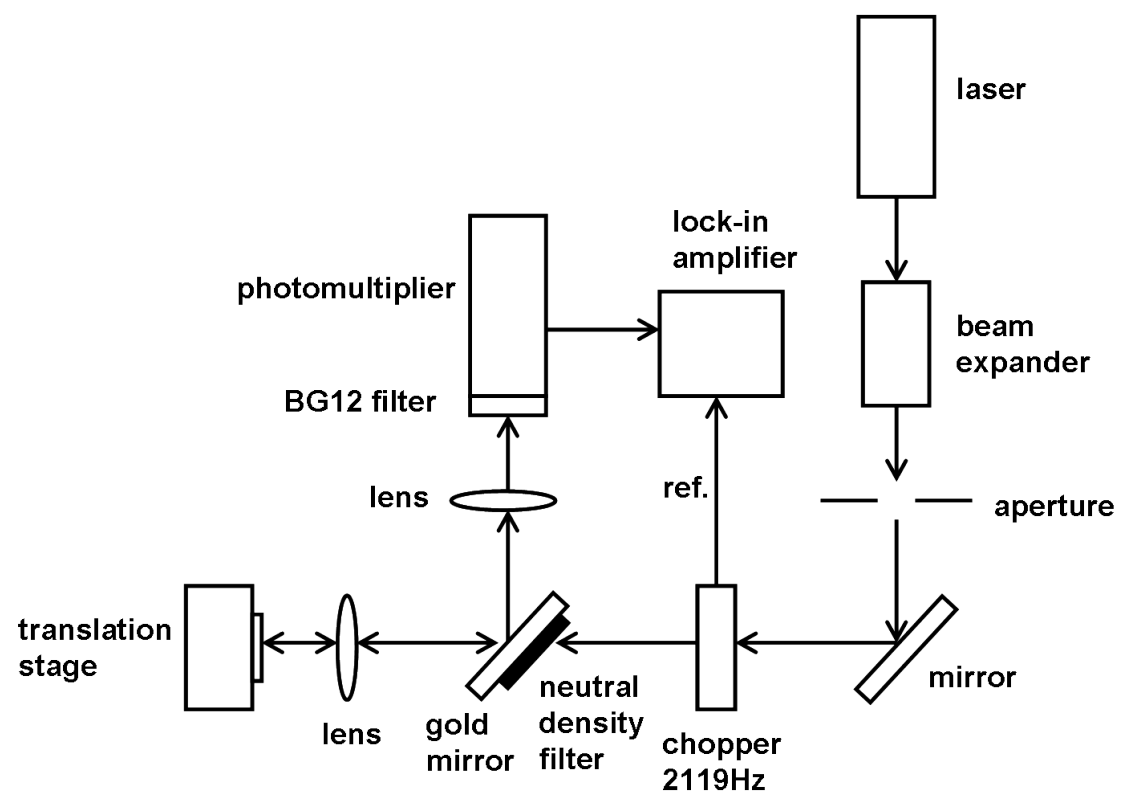

Figure 5.5: Schematic of experimental image read-out system.

gold mirror was made at Victoria University of Wellington (VUW) by depositing a $20 \mathrm{~nm}$ gold layer onto a microscope slide using a gold evaporator. Analysis of the mirror shows the transmittance of the laser light to be approximately $30 \%$. A 0.6 neutral density filter was placed on the rear of the gold mirror to prevent a double spot caused by reflection in the mirror. A BG12 filter is placed between the gold mirror and photomultiplier to ensure only the blue PSL light is detected and scattered red laser light is blocked. All of the PSL light is detected, not just that from the laser spot, making the system comparable to commercial imaging systems.

The signal from the photomultiplier $\left(V_{\text {in }}\right)$ is passed to a Stanford Research Systems SR510 lock-in amplifier, which coherently amplifies the signal based on a reference signal $\left(V_{\text {ref }}\right)$ generated by the chopper. The output signal $\left(V_{\text {out }}\right)$ is maximised by setting the phase difference between the two input signals to zero, so that when

$$
\begin{gathered}
V_{\text {in }}=V_{\text {in }}^{\circ} \cos \omega t \\
\text { and } V_{\text {ref }}=V_{\text {ref }}^{\circ} \cos (\omega t+\phi) \\
\text { then } V_{\text {out }}=A V_{\text {in }}^{\circ} \cos \phi
\end{gathered}
$$




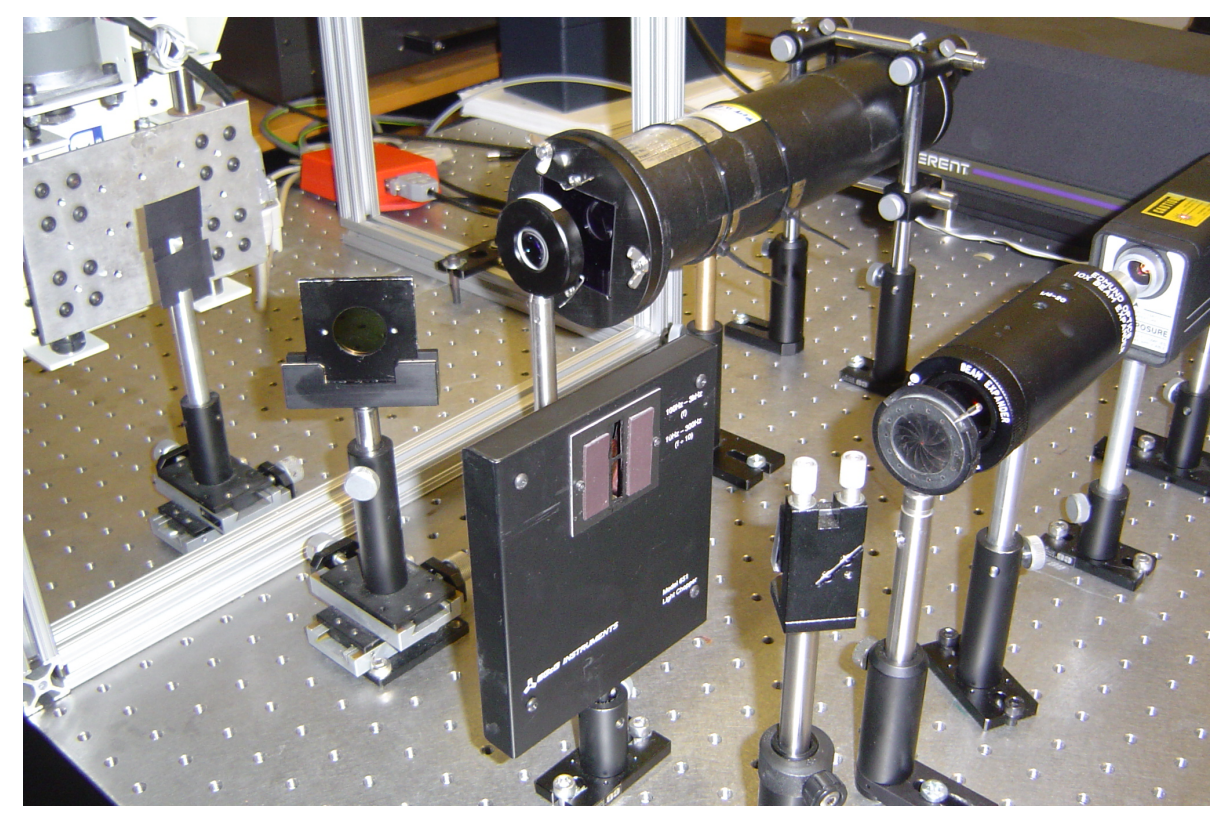

Figure 5.6: Experimental image read-out system.

The image is then stored on a computer by passing the signal from the lock-in amplifier through an analogue to digital (A/D) converter.

\subsubsection{XYZ translation stage}

The translation stage, consisting of $\mathrm{X}, \mathrm{Y}$ and $\mathrm{Z}$ linear positioning tables with MD-2 dual stepper motor systems, was supplied by Arrick Robotics. Movement along all three axes is obtained with the use of stepper motors.

A stepper motor is a device which converts electrical pulses into discrete mechanical movements. Each motor has four coils which create a magnetic field when energised. The rotor is permanently magnetised and reacts with the field so as to cause the motor to rotate. The sequence in which the coils are energised determines the size and direction of the steps; the pattern of coil energisation is referred to as a "phase".

Changing from one phase pattern to another causes the motor to move one step. If the coils are energised but the phase is fixed the motor will stay in the same position. The motors are run in half step mode which corresponds to a step size of $0.9^{\circ}$. The phase pattern for the motors in this mode is given in table 


\begin{tabular}{lllll}
\hline$\#$ & 4 & 3 & 2 & 1 \\
\hline 1 & 1 & 1 & 1 & 0 \\
2 & 1 & 1 & 0 & 0 \\
3 & 1 & 1 & 0 & 1 \\
4 & 1 & 0 & 0 & 1 \\
5 & 1 & 0 & 1 & 1 \\
6 & 0 & 0 & 1 & 1 \\
7 & 0 & 1 & 1 & 1 \\
8 & 0 & 1 & 1 & 0 \\
\hline
\end{tabular}

Table 5.1: Half step phase pattern.

\section{1.}

Rotational motion is converted to translational motion in two different ways (Figure 5.7). For the $X$ and $Y$ direction a pulley and belt drive is used, the resolution (step size) is then a function of the diameter of the pulley. In the $Z$ direction a lead-screw drive is used which allows for a greater resolution than a pulley and belt drive, but the motors have to be run at a slower speed. The resolution of the lead-screw drive depends on the pitch of the lead screw. The translation stage is capable of $127 \mu \mathrm{m}$ steps in the $\mathrm{X}$ and $\mathrm{Y}$ directions and 2.5 $\mu \mathrm{m}$ in the $\mathrm{Z}$ direction in half step mode; the high resolution in the $\mathrm{Z}$ direction was achieved through the use of a custom lead-screw cut in the VUW workshop.

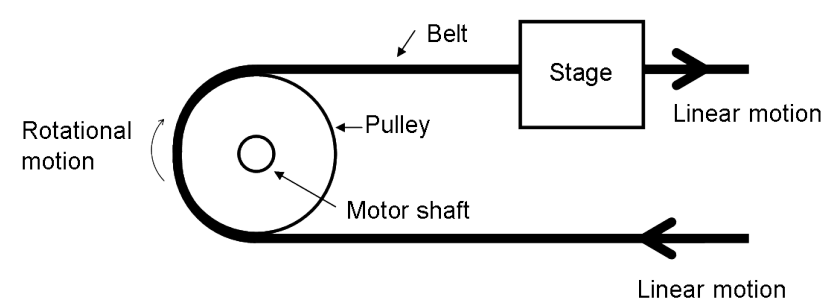

(a) Pulley and Belt drive.

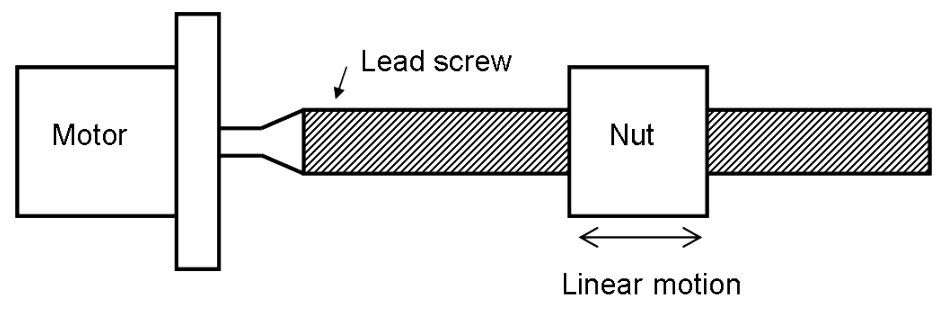

(b) Lead-screw drive.

Figure 5.7: Converting rotational motion into translational motion. 
Each stepping motor has a micro-switch which is used to find the "home" position. Home positioning works by stepping the motor in reverse until the switch is pressed, then moving the motor forwards until the switch is first released. When the switch is pressed the input to the control unit is connected to ground, otherwise it is at $+5 \mathrm{~V}$.

The translation stage is supplied with MD2 control units from Arrick Robotics, each of which can control up to two motors. The MD2 control units supply power to the motors, and the motors and switches can be controlled or monitored with a computer via the parallel printer port on the rear of the box.

\subsubsection{Computer interface}

A SuperLogics ADC-1 analogue to digital converter is used to control the experiment by a computer via an RS-232 port. The ADC-1 contains eight 12 bit analogue acquisition lines and sixteen 16 bit digital lines, each of which can be defined by the user. To send and receive data from the ADC-1 simple ASCII commands are used, with numerical data being represented by hexadecimal integers. Command examples are given in table 5.2.

Twelve of the digital lines are used as outputs from the ADC- 1 to drive the stepper motors and three are used as inputs from the translation stage to monitor switches. The command for the digital lines has five parts; the first tells the box what it is to do, the second monitors the switches and the last three control motors 3 to 1 respectively. One analogue line is used to receive the signal from the lock-in amplifier. The signal received from the analogue line is converted

\begin{tabular}{cc}
\hline Command & Description \\
\hline Txxxx & Set digital direction \\
Gxxxx & Get digital direction \\
Ixxxx & Input digital direction \\
Oxxxx & Output digital direction \\
Ux & Unipolar analogue input \\
\hline
\end{tabular}

Table 5.2: Commands for SuperLogics ADC-1, $\mathrm{x}$ is a hexadecimal integer. 


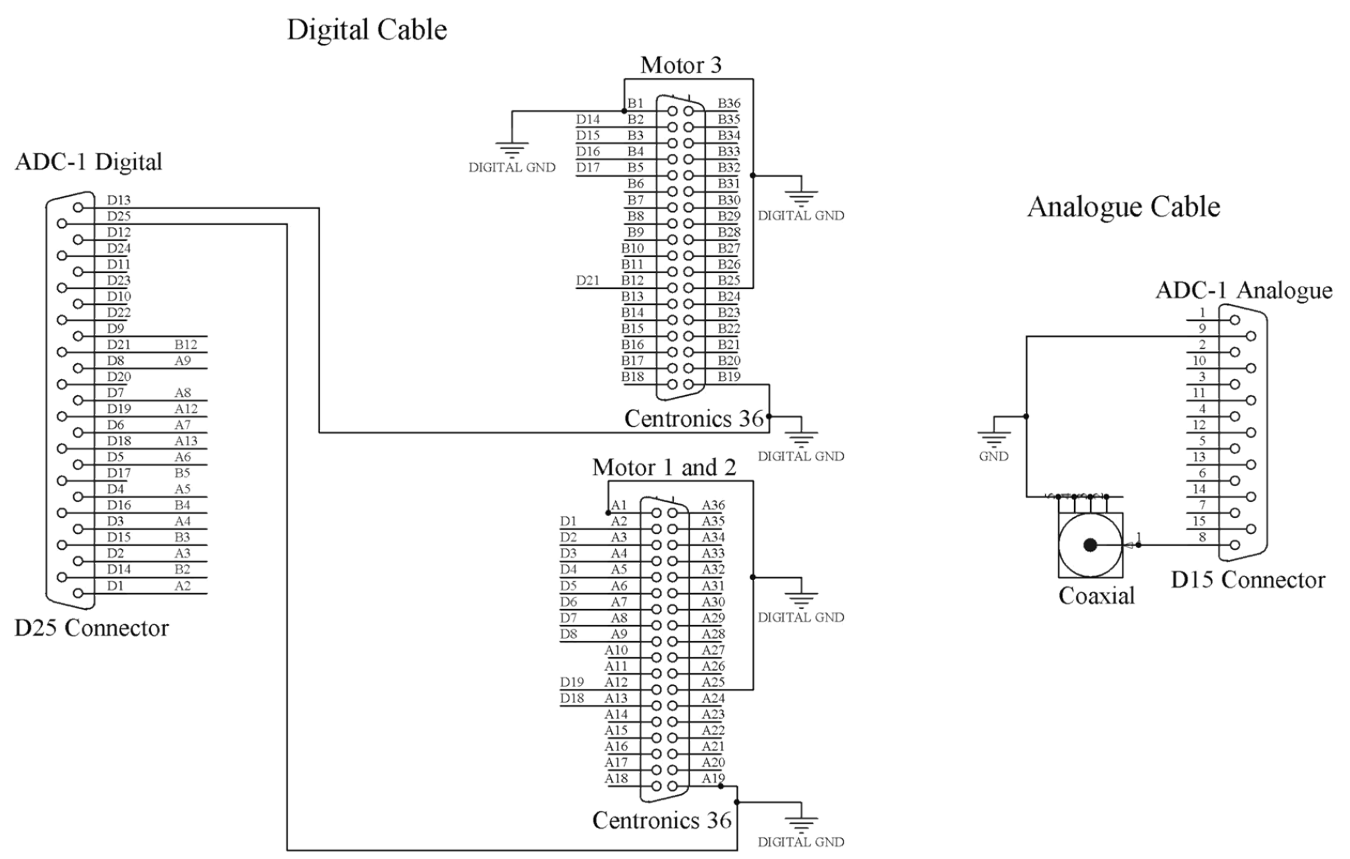

Figure 5.8: Circuit diagram of cables for SuperLogics ADC-1 box. For the digital cables the right D25 connector is connected to the digital port of the ADC-1 and the middle centronics are connected to the motors. The two sets of numbers represent the wiring of the cables, for example D1 A2 refers to line 1 of the D25 being connected to line 2 of Motors 1 and 2 centronics pin. One analogue line is connected to a coaxial cable.

to a voltage an the basis of equation 5.6 where $V_{\text {ref }}$ is the maximum voltage for the ADC-1. Figure 5.8 shows the circuit diagram of the cables connecting the computer interface to the translation stage and lock-in amplifier.

$$
\text { Volts }=\text { Sample } * \frac{V_{\text {ref }}}{4096}
$$

The ADC-1 is controlled by a LabVIEW program which drives the stepper motors and records the signal from the lock-in amplifier as a function of distance across the sample, as described below.

\subsubsection{LabVIEW program}

LabVIEW is a development environment and platform for a visual programming language known as " $G$ " developed by National Instruments. LabVIEW can be used for applications such as data acquisition and instrument control. 
" $G$ " is a dataflow programming language where the execution of the program is determined by the structure of a graphical diagram on which the different functions are connected together with wires. LabVIEW programs and subprograms are known as VI's ("virtual instruments").

Figure 5.9 and 5.10 show the block diagram of the LabVIEW program used to control the experiment. At the top of figure 5.9 is code to control stepping of the motors. Each motor can be moved a given number of steps in either forwards or reverse direction with a set delay time between each step. This works by reading the phase position the motor is at through the ADC-1, then determining the next phase pattern and sending it to the motor via the ADC-1. This is repeated until the given number of steps have been reached. The bottom of this figure shows the code to control the home positioning of each direction of the translation stage. First the motor is stepped backwards until the switch is pushed using the same code described before. The program decides if the switch is pushed by monitoring the string and stopping when the string read matches the string corresponding to a switch being pressed. Once the switch has been pushed the motor then steps forward until the switch is no longer pressed.

Figure 5.10 is the code to control the scanning program, where the motor is stepped in a given direction and the signal is read from the lock-in amplifier. First the timer is initialised, otherwise the first steps are at a faster rate than that desired. The motor is stepped through a number of steps before a reading from the lock-in is taken. The number of steps per measurement is normally set at one. To read the signal from the lock-in, a command is sent to the ADC-1 which responds with the given voltage in hexadecimal form. The hexadecimal integer is converted to the equivalent voltage using equation 5.6. This is repeated until the total number of steps have been reached. For each measurement the position of the motor and the intensity from the lock-in is plotted on a graph and saved to a file. 


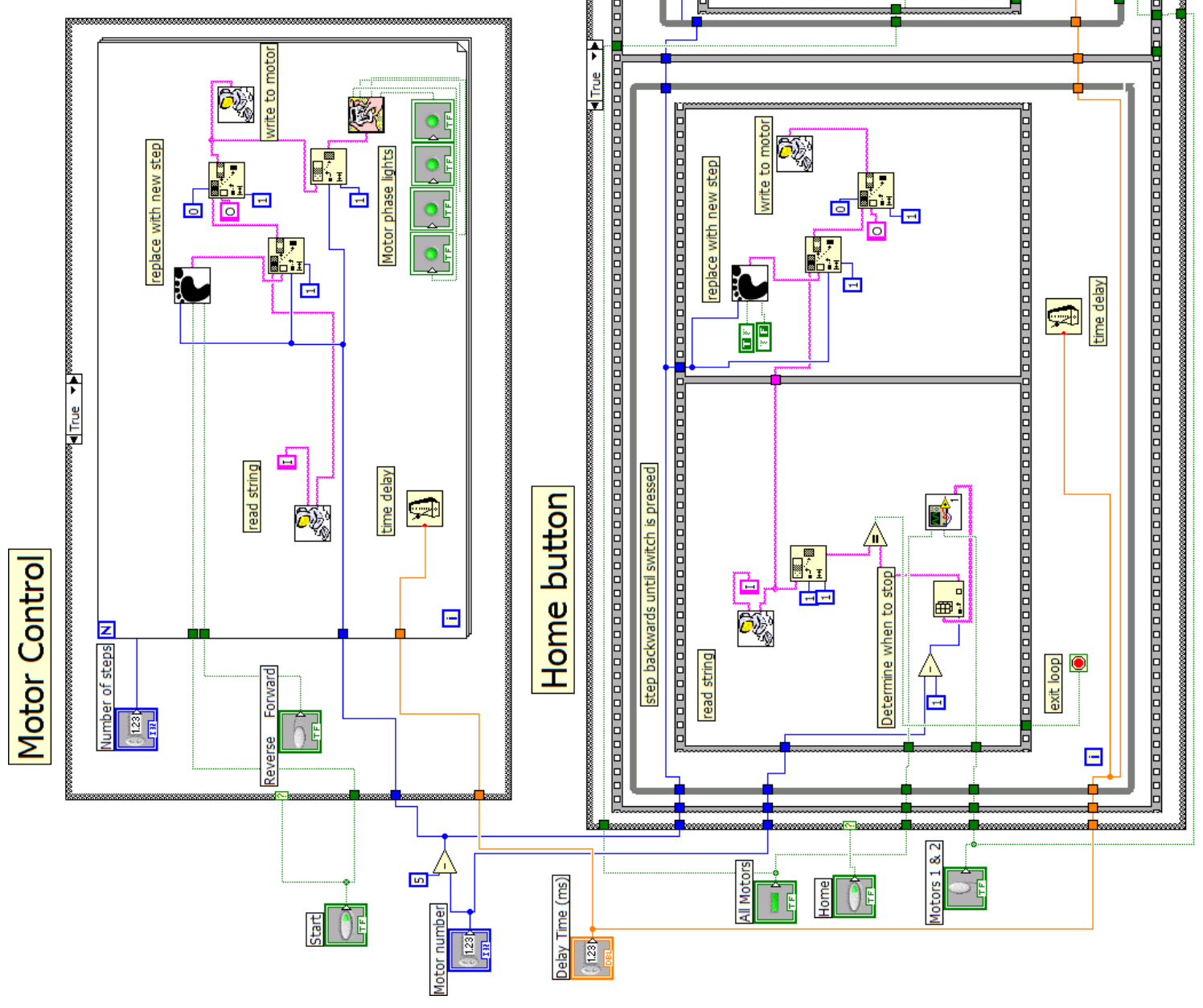

Figure 5.9: LabVIEW block diagram for controlling stepping of the motors and the home switch. 


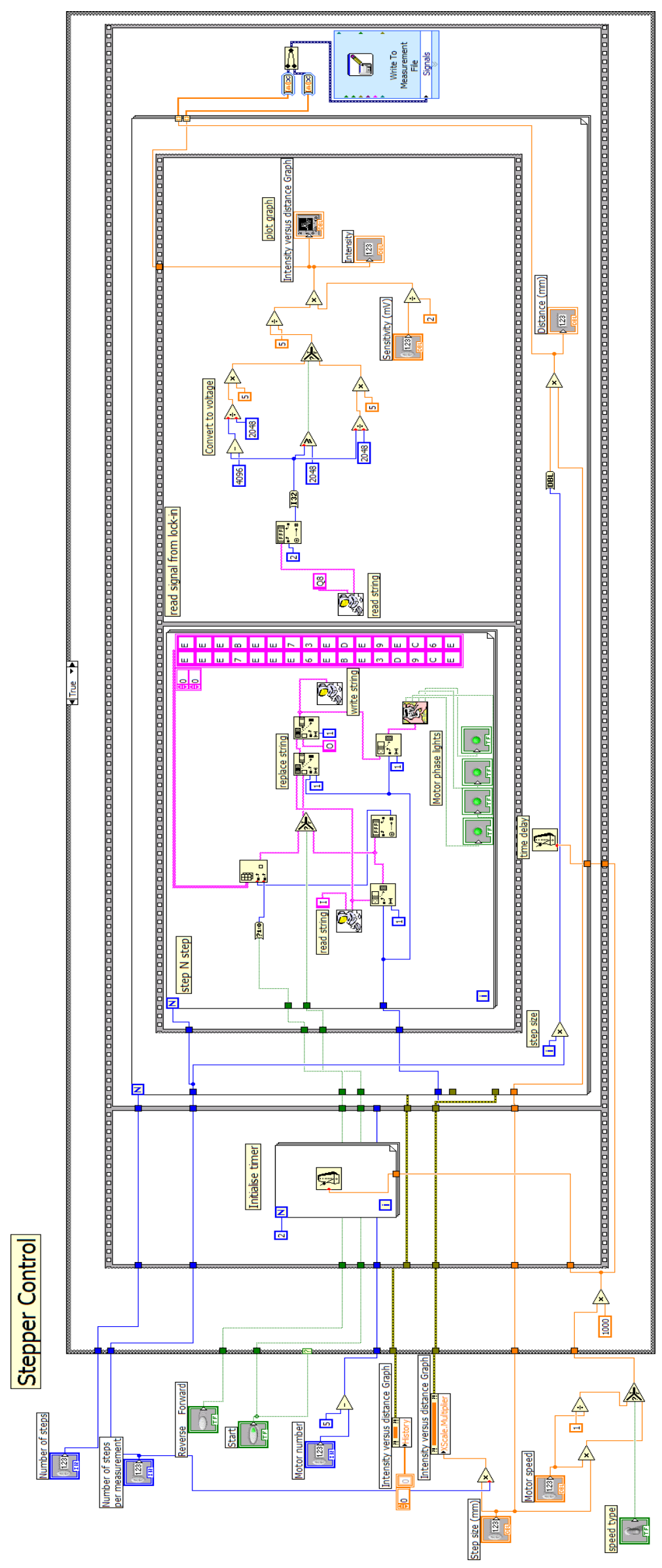

Figure 5.10: LabVIEW block diagram for controlling the scanning program. 


\section{Chapter 6}

\section{Experimental Results}

\subsection{Read-out laser}

The beam diameter of the He-Ne laser incident on the plate was determined by scanning the laser across a Gammex MA0647 gold test pattern and recording the reflected red light. The gold grid consists of groups of gold-nickel alloy lines mounted on an acrylic wafer where the spatial resolution of the groups range from $5 \mathrm{lp} / \mathrm{mm}$ to $20 \mathrm{lp} / \mathrm{mm}$. An example of a scan across part of the gold grid can be seen in figure 6.1. The first step on the far left is caused by a $2 \mathrm{~mm}$ wide band of gold and the subsequent blocks of 5 lines correspond to spatial frequencies starting at $5 \mathrm{lp} / \mathrm{mm}$ and ranging to $20 \mathrm{lp} / \mathrm{mm}$.

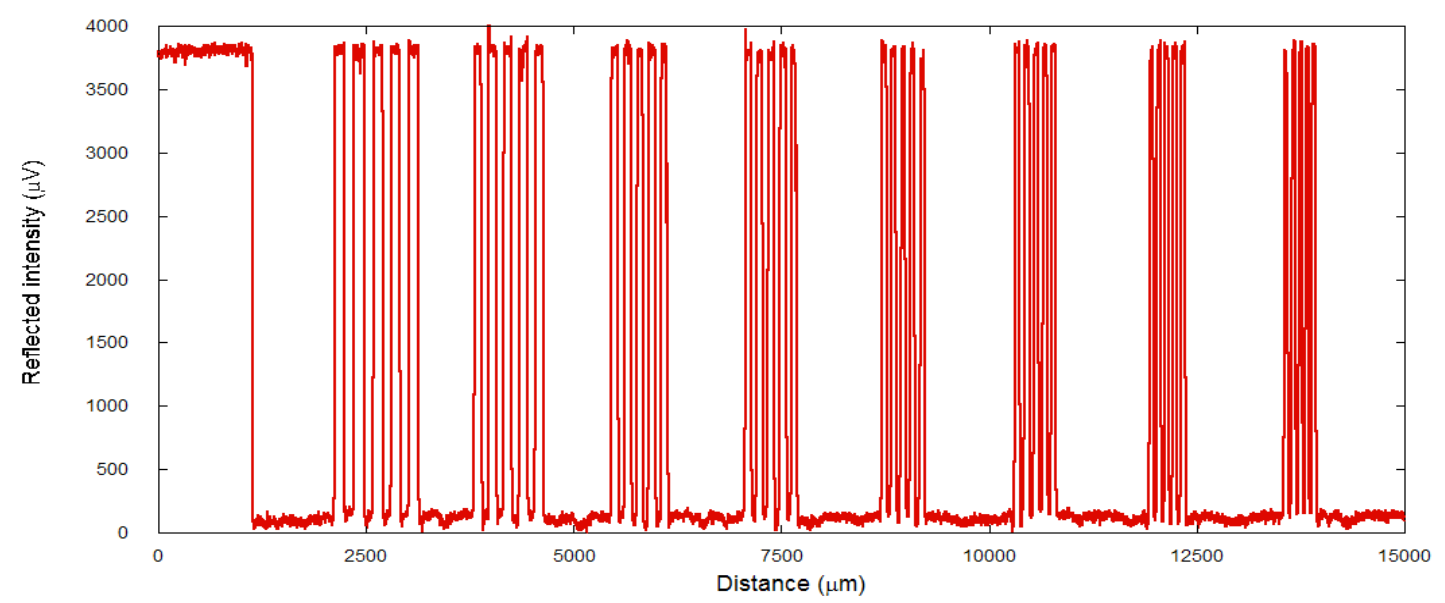

Figure 6.1: Image of gold grid obtained by scanning laser across the grid and recording the reflected red light. 


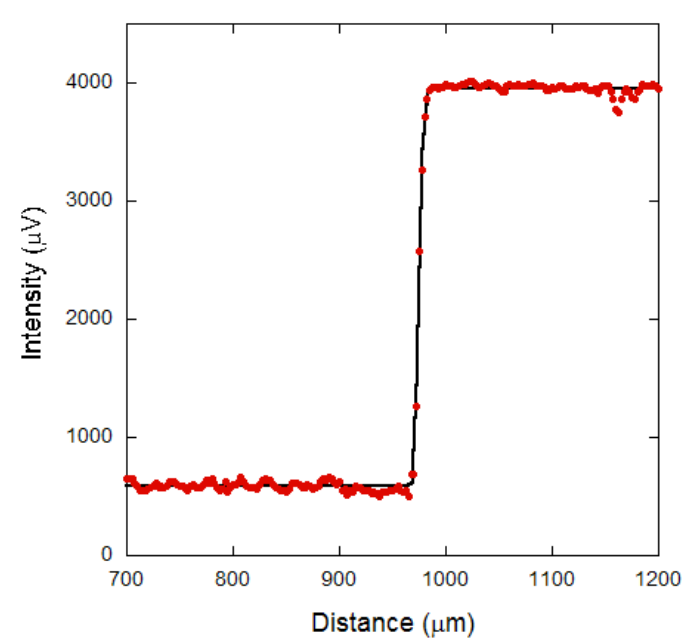

(a)

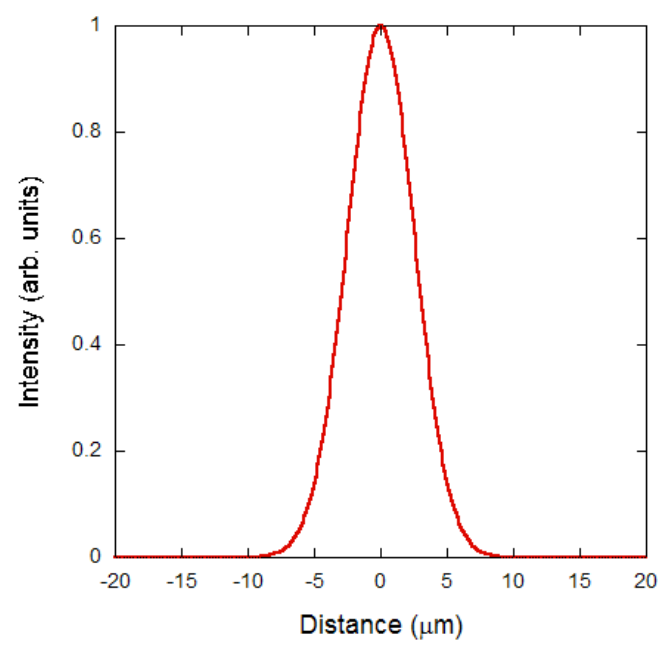

(b)

Figure 6.2: (a) Image of sharpest edge of gold grid fitted with an error function. (b) Profile of laser spot in one dimension.

To find the variance of the laser beam the sharpest edge was found and assuming the laser beam has a Gaussian form an error function (equation 6.1) was fitted to the edge by least square fitting methods using Kaleidagraph.

$$
\operatorname{erf}\left(\frac{x}{\sqrt{2} a}\right)=\int_{0}^{x} \exp \left(\frac{-t^{2}}{2 a^{2}}\right) d t
$$

An error function is used to represent the edge to ensure that after differentiating and/or Fourier transforming, a Gaussian form (required for the LSF and MTF) is obtained. It is an empirical approximation which is justified by the good fit.

The sharpest edge is chosen to determine the spot size because there are many reasons which will cause a broadening of the edge (for example scattering) but none except the profile of the laser which will lead to a sharpening of the edge. The sharpest edge can be seen in figure 6.2(a), where curve fitting determined the variance of the laser (parameter $a$ ) to be $3.0 \mu \mathrm{m}$. The profile of the laser in one dimension is found by differentiating the error function with respect to $x$ (equation 6.2), and is shown in figure 6.2(b).

$$
I(x)=\exp \left(\frac{-x^{2}}{2 a^{2}}\right)
$$




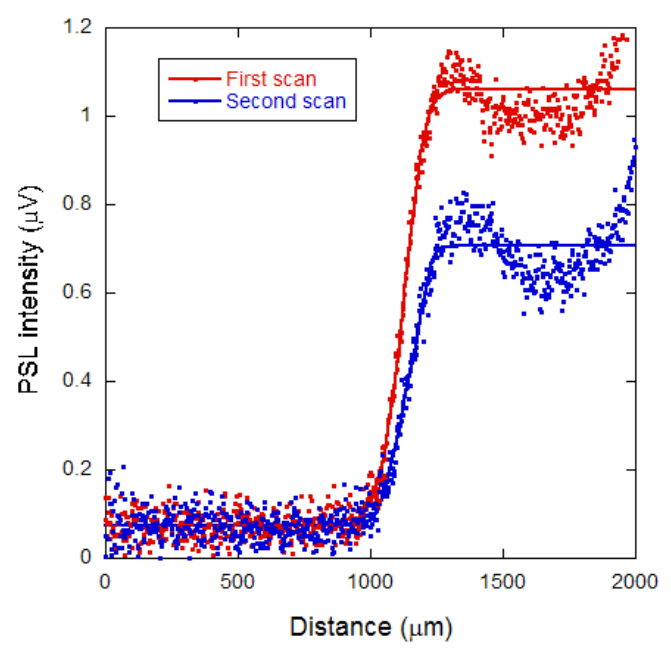

Figure 6.3: Two consecutive scans of an edge stored on an Agfa MD30 imaging plate.

To determine the power of the laser incident on the plate, the initial power and intensity were measured using a power meter and photomultiplier respectively, and these were compared to the intensity after travelling through all the optical components. Assuming the relationship between laser power and intensity is linear, the power incident on the plate can be determined with the use of equation 6.3.

$$
\frac{P_{\mathrm{i}}}{I_{\mathrm{i}}}=\frac{P_{\mathrm{f}}}{I_{\mathrm{f}}}
$$

The power of the laser spot incident on the plate was determined to be $2 \mu \mathrm{W}$. This laser power causes $30 \%$ of the image to be read-out in each scan, as can be seen in figure 6.3 which shows two consecutive scans on an Agfa MD30 imaging plate irradiated through a lead foil to give an edge.

\subsection{Agfa MD30 imaging plate}

The Agfa MD30 imaging plate is an older generation commercial imaging plate which consists of $\mathrm{Ba}_{1-x} \mathrm{Sr}_{x} \mathrm{FBr}_{1-w} \mathrm{I}_{w}$ :Eu crystals embedded in an organic binder, with typical luminescence at $400 \mathrm{~nm}$. These have been replaced by new MD4.0 plates which have an additional adhesion layer, improved phosphor layer and a slightly higher resolution. An illustration of the MD4.0 plate 


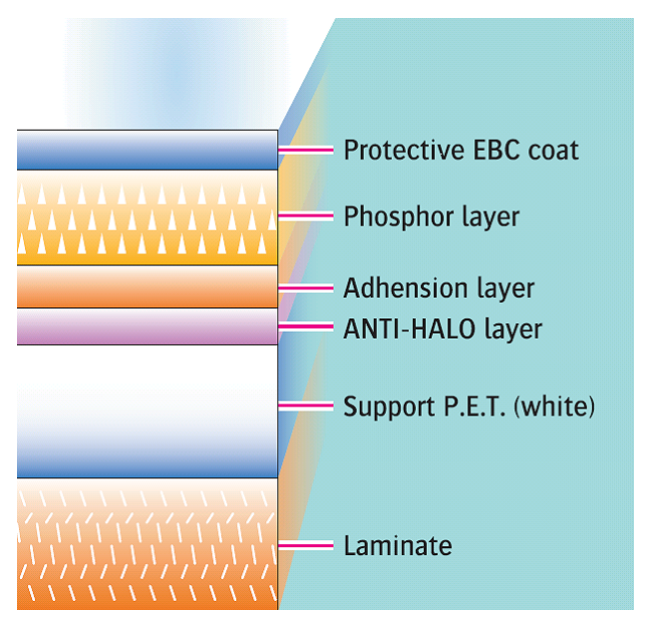

Figure 6.4: Structure of the Agfa MD4.0 general plate [30].

structure can be seen in figure 6.4 with the only difference in the MD30 plate is the absence of the adhesion layer. The anti-halo layer is a specially designed Agfa-patented blue layer which absorbs the laser light but allows through the stimulated light.

The scattering length of the imaging plate was found by the process explained in section 5.2. Firstly the sample was prepared by removing the backing and anti-halo layers, and then polishing both sides until the sample was at a thickness of approximately $20 \mu \mathrm{m}$. Thinning the sample down allows some light to be transmitted through the sample. It was assumed that no absorption occurs in the sample and $6 \%$ of light was found to be reflected off the surfaces using a refractive index of 1.65 [29] for $\mathrm{BaFBr}$.

The graph produced by the spectrophotometer of absorbance versus wavelength is transformed into one of scattering length versus wavelength with the use of equation 6.4, which is a version of equation 5.3 assuming no absorption.

$$
\mu=\frac{z \log _{10}(e)}{A b s(\lambda)+\log _{10}(1-R)^{2}}
$$

Figure 6.5 shows the graph of scattering length versus wavelength. At low wavelengths the large fluctuations in scattering length are caused by fluctuations in the intensity of the deuterium lamp. The scattering length is approximately $3 \mu \mathrm{m}$ for both red laser light at $633 \mathrm{~nm}$ and PSL light at $390 \mathrm{~nm}$. 


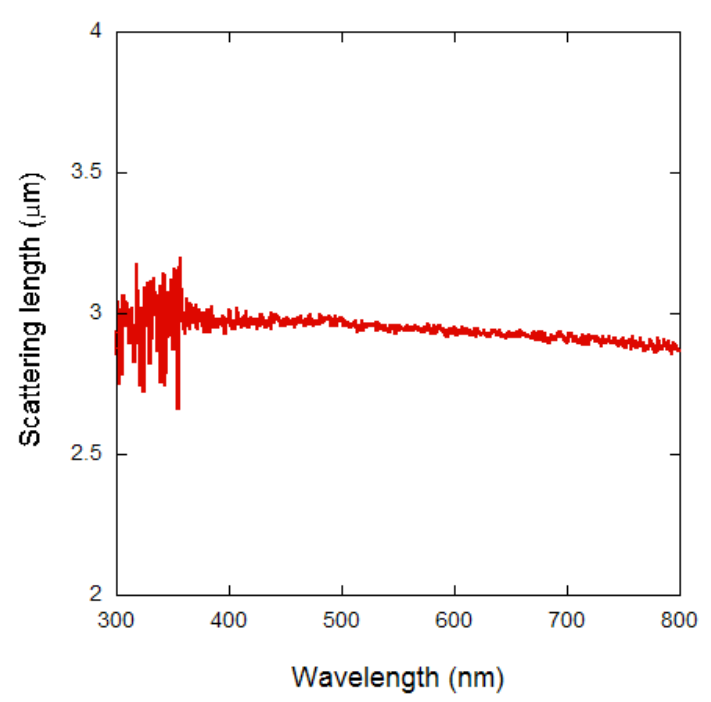

Figure 6.5: Scattering length versus wavelength for Agfa MD30.

Absorption by the material was assumed to be zero but some of the red laser light is absorbed by storage centres in order for the electrons or holes to overcome their traps. The optical cross-section for photostimulation $(\sigma)$ and the density of storage centres $\left(n_{\mathrm{sc}}\right)$ are used to find the absorption coefficient for red light with the use of equation 6.5. The values were taken to be $\sigma=8.85 \times$ $10^{-20} \mathrm{~m}^{2}$ and $n_{\mathrm{sc}}=1.0 \times 10^{21} \mathrm{~m}^{-3}$ from the literature [17] which leads to an absorption coefficient of $\gamma=8.85 \times 10^{-5} \mu \mathrm{m}^{-1}$.

$$
\gamma=n_{\mathrm{sc}} \sigma
$$

A scanning electron microscope (SEM) was used to determine the size of the $\mathrm{BaCl}_{2}$ particles in the Agfa MD30 imaging plate. The SEM creates an image of the surface of the sample by scanning across the sample with a beam of highenergy electrons. A cross-sectional area of the plate was imaged and can be seen in figure 6.6. There is a wide dispersion of particle sizes; the average particle size was taken to be $8 \mu \mathrm{m}$ by averaging over 20 particles.

The MTF was found using both the edge method and test pattern method. Both objects were irradiated at $40 \mathrm{kV}$ and $0.3 \mathrm{~mA}$ for 30 minutes at a source to object distance of $460 \mathrm{~mm}$ in the Faxitron. After read-out of the image the 


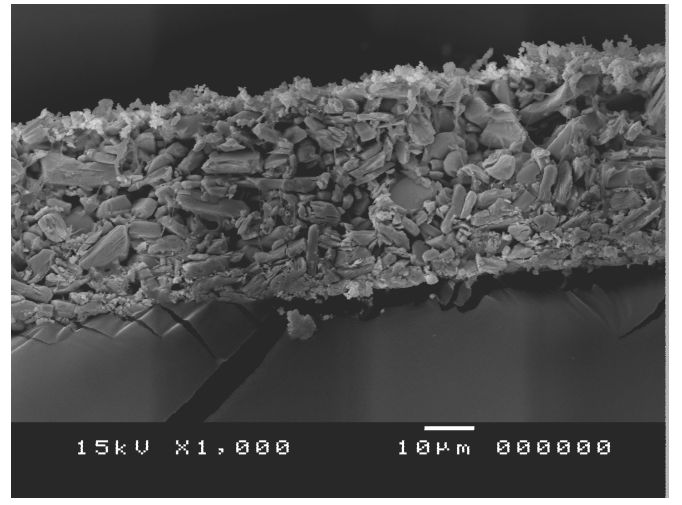

(a)

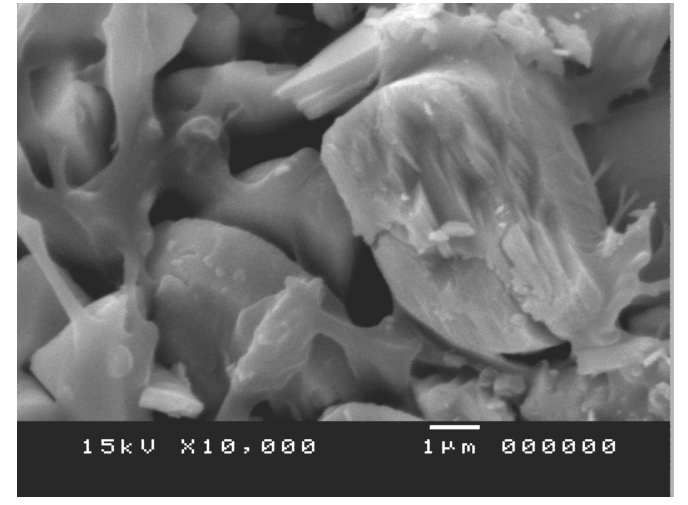

(b)

Figure 6.6: SEM images of cross-section of Agfa MD30 imaging plate. The dark bands in figure (a) are caused by electronic noise.

sample was bleached for 10 minutes.

A Cardinal Health 07-553 lead grid test pattern was used which ranges in spatial frequencies from $0.25 \mathrm{lp} / \mathrm{mm}$ to $10 \mathrm{lp} / \mathrm{mm}$ with a lead thickness of 0.05 $\mathrm{mm}$. The object had to be irradiated in two stages as its length was greater than the travel distance of the positioning table.

The image of the lead grid with the varying spatial frequencies can be seen in figure 6.7. To find the MTF, the image is split up into each spatial frequency and least squares fitted with a sine wave in the form of equation 6.6, where $x$ is the distance and $\nu$ is the spatial frequency.

$$
f(x)=A \sin (2 \pi \nu x)
$$

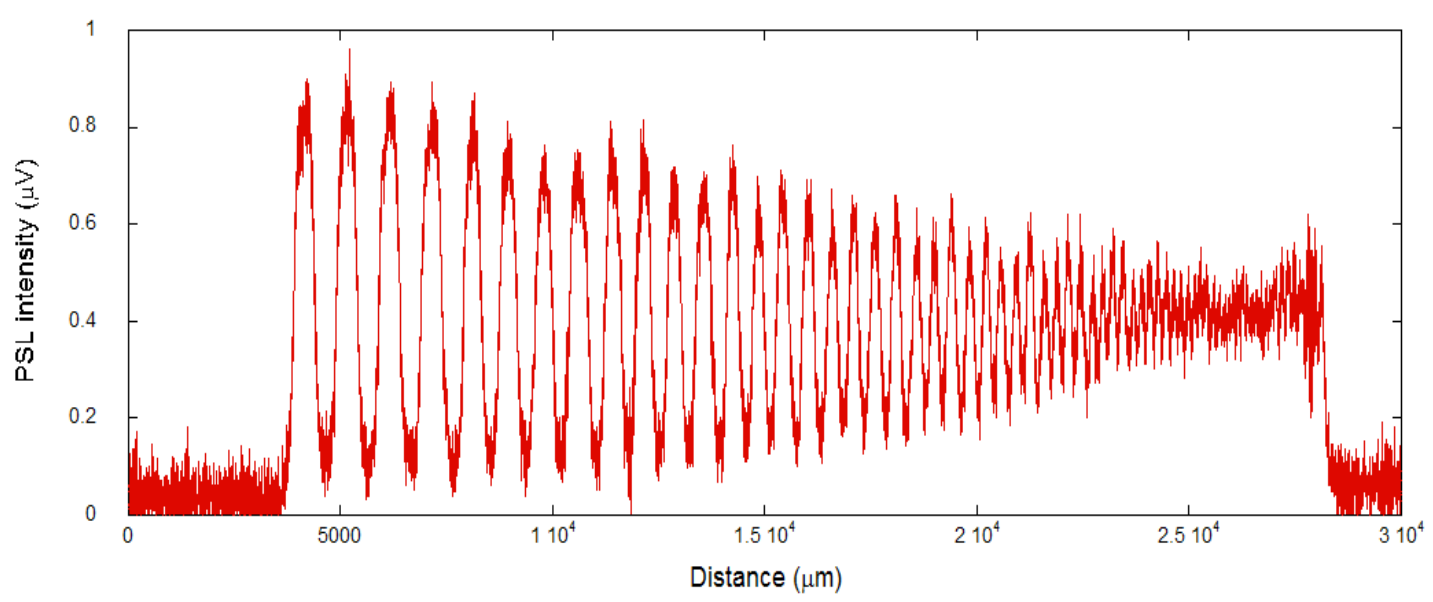

Figure 6.7: Image of lead grid read-out from Agfa MD30. 
The amplitude $A$, of the sine wave is then equal to the unnormalised MTF value at that particular spatial frequency. A graph of spatial frequency versus MTF is then normalised to 1 for a spatial frequency of zero. To image the full range of spatial frequencies the lead grid object had to be irradiated twice as the object was larger than the travel distance of the experimental equipment. Irradiating the object twice meant the overlapping spatial frequencies had different amplitudes and the first image had to be normalised to the second with respect to the overlapping spatial frequencies. The MTF found by using the grid method can be seen as the red circles in figure 6.8(b). The resolution of the plate using this method is $4.5 \mathrm{lp} / \mathrm{mm}$ at an MTF equal to 0.2 .

The second method involves an image of a sharp edge which was created by cutting a $150 \mu \mathrm{m}$ thick lead foil with a guillotine. The image of this edge can be seen in figure 6.8(a). Two error functions are least square fitted to the edge in the form of equation 6.7, where the parameters $a, b, A$ and $B$ are found to be $45.1 \mu \mathrm{m}, 88.9 \mu \mathrm{m}, 0.224 \mu \mathrm{m}$ and $0.463 \mu \mathrm{m}$ respectively.

$$
\operatorname{ESF}(x)=A \operatorname{erf}\left(\frac{x}{\sqrt{2} a}\right)+B \operatorname{erf}\left(\frac{x}{\sqrt{2} b}\right)
$$

The ESF was fitted with two error functions to achieve the best fit and to allow

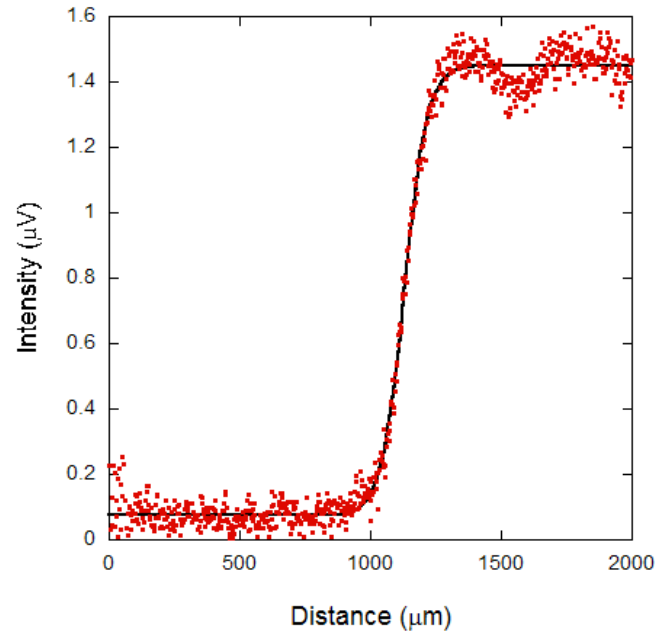

(a)

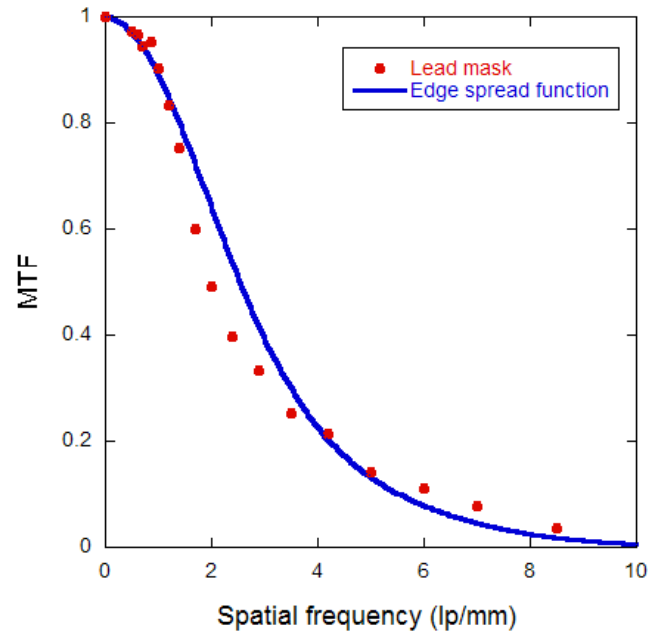

(b)

Figure 6.8: (a) ESF and (b) MTF for Agfa MD30 imaging plate. 
for the possibility of a bimodal MTF.

The MTF curve for the edge method can be seen as the blue line in figure 6.8(b) and is found by inserting $a, b, A$ and $B$ into equation 6.8 , which is the result of differentiating equation 6.7 and then taking the Fourier transform.

$$
M T F(x)=A \exp \left(-2 \pi^{2} a^{2} x^{2}\right)+B \exp \left(-2 \pi^{2} b^{2} x^{2}\right)
$$

Again the resolution at a MTF equal to 0.2 is $4.5 \mathrm{lp} / \mathrm{mm}$. There is quite reasonable agreement between the two methods as can be seen from figure 6.8(b) with the two methods showing slightly different MTF's but both have an overall Gaussian nature which corresponds to a short scattering length as seen in section 4.2. At very low frequencies the two curves are in very close agreement and for spatial frequencies around $1.5 \mathrm{lp} / \mathrm{mm}$ to $4.5 \mathrm{lp} / \mathrm{mm}$ the MTF curve for the grid method is lower than for the edge method. At high spatial frequencies the grid method MTF is larger than for the edge method which reflects the inability of the edge method to distinguish high spatial frequencies.

\section{$6.3 \quad \mathrm{~N} 05-02-50$ glass ceramic}

The N05-02-50 glass ceramic sample was provided by Dr P.J. Newman from the Chemistry department at Monash University, Melbourne. The composition of the glass is $53 \mathrm{ZrF}_{4}, 20 \mathrm{BaCl}_{2}, 20 \mathrm{NaF}, 3 \mathrm{LaF}_{3}, 3 \mathrm{AlF}_{3}, 2 \mathrm{InF}_{3}$ and $1 \mathrm{EuCl}_{2}$ in mole percent. The glass was prepared by melting the fluorides at $800{ }^{\circ} \mathrm{C}$ for 1 hour, cooling to room temperature and then the chlorides are added and melted at $750{ }^{\circ} \mathrm{C}$ for 1 hour. The glass is then poured and annealed for 2 hours at $200{ }^{\circ} \mathrm{C}$ before being cooled to room temperature over 16 hours. The crystallisation of $\mathrm{BaCl}_{2}$ varied over the sample, as did the thickness of the sample.

The scattering length was again determined by the procedure explained in section 5.2 and using equation 6.4. Absorption of the light by the sample was assumed to be zero and the reflection off surfaces was calculated to be $4 \%$ using 


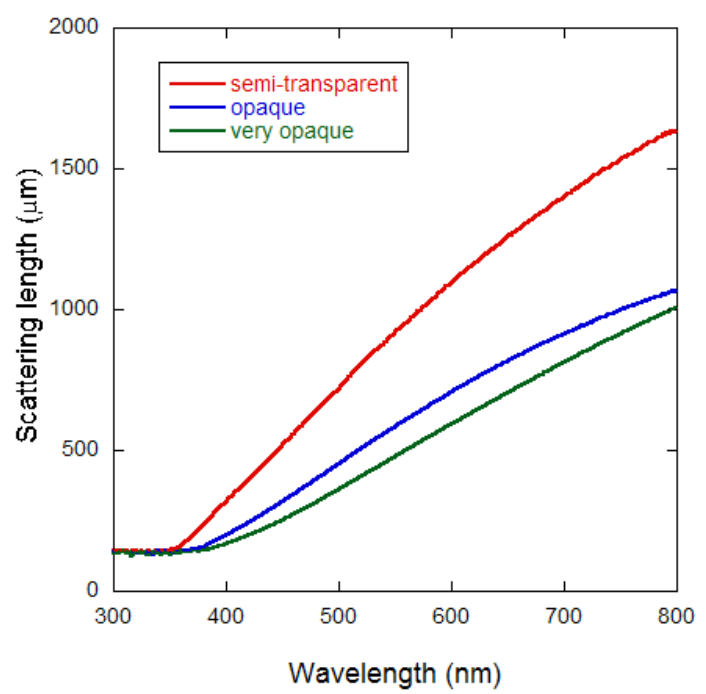

Figure 6.9: Scattering length versus wavelength for the various crystallisation regions of N05-02-50 glass ceramic sample as shown.

a refractive index of 1.498 [31] for ZBLAN.

Due to variations in the crystallisation the scattering length was determined in three separate locations, from near-transparent to very opaque and can be seen in figure 6.9. The thickness of the sample also varied with a difference of $200 \mu \mathrm{m}$ from the thinnest to thickest part. The average thickness of the sample was taken to be $1230 \mu \mathrm{m}$.

The scattering length was found to range from $666 \mu \mathrm{m}$ to $1205 \mu \mathrm{m}$ for red laser light and from $153 \mu \mathrm{m}$ to $278 \mu \mathrm{m}$ for PSL light, with the lower values coming from the very opaque region.

Absorption of the laser light by storage centres is assumed to be the same as for the Agfa plate $\left(\gamma=8.85 \times 10^{-5} \mu \mathrm{m}^{-1}\right)$, as the optical cross-section of photostimulation and the density of centres are not readily available.

X-ray diffraction (XRD) patterns (figure 6.10) of the glass recorded on a Phillips diffractometer using a $\mathrm{Cu}$ tube, show a predominantly hexagonal $\mathrm{BaCl}_{2}$ phase with particle diameters of $16 \mathrm{~nm}$. This estimate was based on the Scherrer relationship [32] (equation 6.9 where $\beta$ is the width of the line) and choosing the 


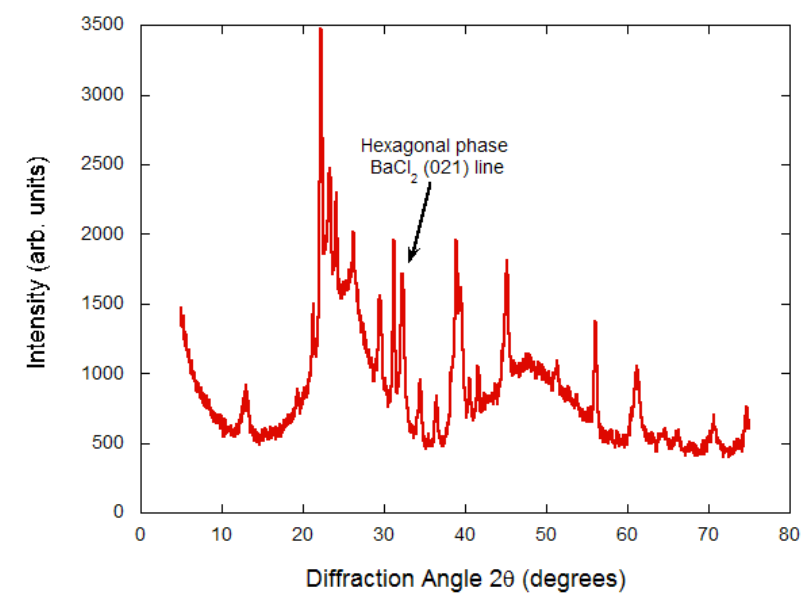

Figure 6.10: XRD pattern of the N05-02-50 glass ceramic sample.

(021) reflection for minimal overlap with other lines.

$$
d=\frac{0.9 \lambda}{\beta \cos \theta}
$$

Additional XRD lines to those expected for the hexagonal $\mathrm{BaCl}_{2}$ phase are attributed to a small fraction of the orthorhombic phase and some unidentified surface phase.

The MTF was determined using the edge and grid method as explained in section 5.4, with the same analysis as given in the previous section. The objects were irradiated at $50 \mathrm{kV}$ and $0.3 \mathrm{~mA}$ for 1 hour in the Faxitron with the distance between object and source being between 50 and $100 \mathrm{~mm}$. After the image was read-out the samples were bleached for 10 minutes.

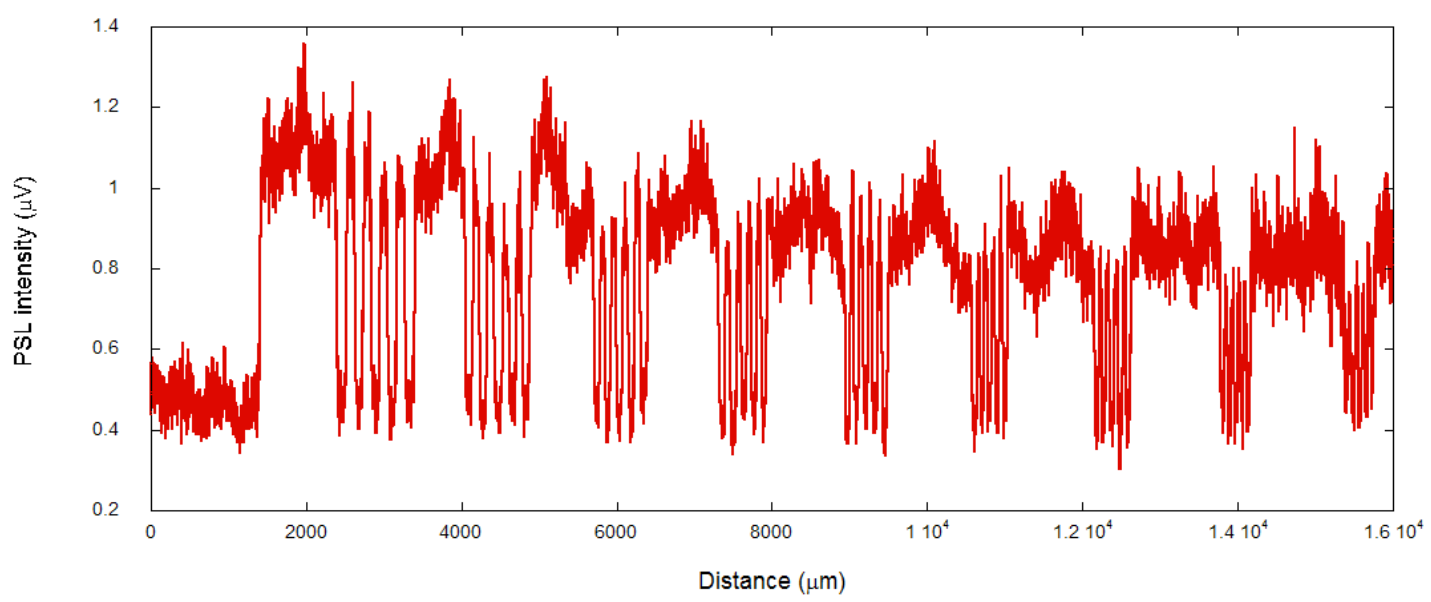

Figure 6.11: Image of gold grid produced by N05-02-50 glass ceramic. 


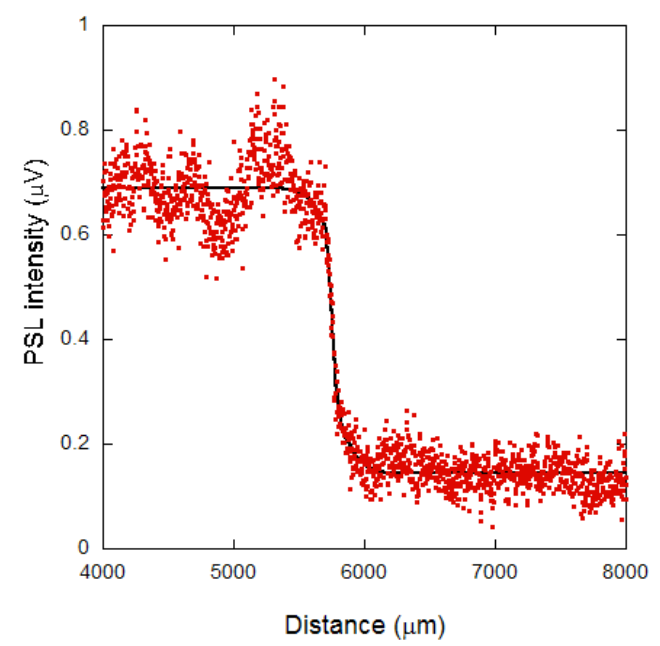

(a)

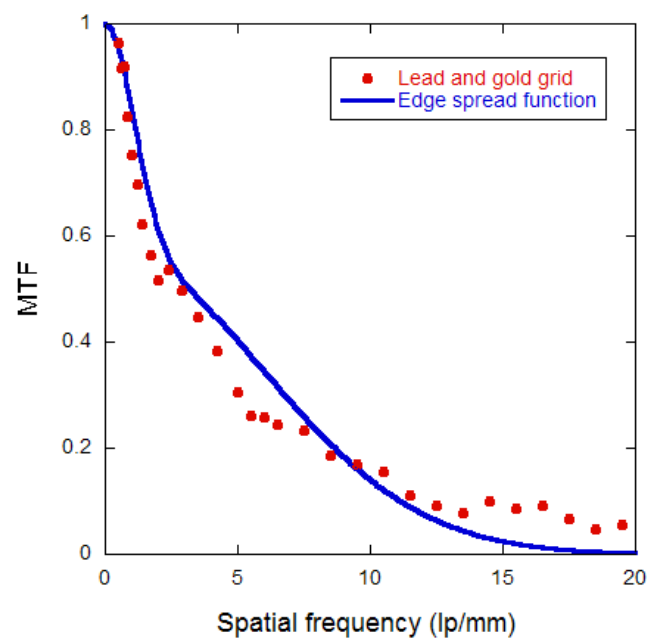

(b)

Figure 6.12: (a) ESF and (b) MTF for N05-02-50 glass ceramic.

In addition to the lead grid, the gold grid was also used to extend the measurements up to higher spatial frequencies. The pattern produced by the gold grid is slightly different, seen in figure 6.11, as the lines have a lower intensity than the background. From the manufacturers data sheet the gold grid ranges in spatial frequencies from $5 \mathrm{lp} / \mathrm{mm}$ to $20 \mathrm{lp} / \mathrm{mm}$ but analysis showed that the actual values are $4.5 \mathrm{lp} / \mathrm{mm}$ to $19.5 \mathrm{lp} / \mathrm{mm}$.

Again, to determine the MTF using the grid method the sample and object had to be irradiated several times (four for the lead grid and two for the gold grid) because of the small size of the sample. Due to the small size it was not al-

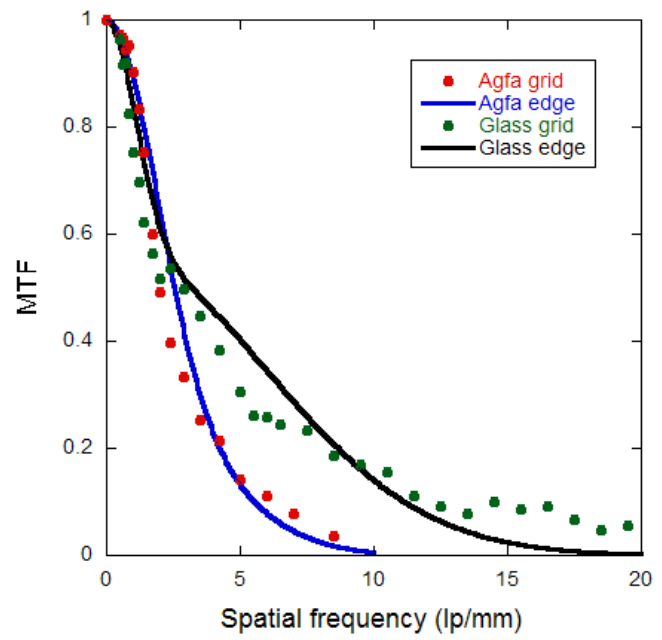

Figure 6.13: MTF's for both the glass ceramic sample and Agfa MD30 imaging plate. 
ways possible to overlap the spatial frequencies; the joining of the images was guided by the MTF found for the edge method.

The MTF for both methods and the edge are shown in figure 6.12. Fitting two error functions to the edge give values of $26.6 \mu \mathrm{m}, 152.2 \mu \mathrm{m}, 0.156 \mu \mathrm{m}$ and $0.117 \mu \mathrm{m}$ for the parameters $a, b, A$ and $B$ respectively. As with the Agfa

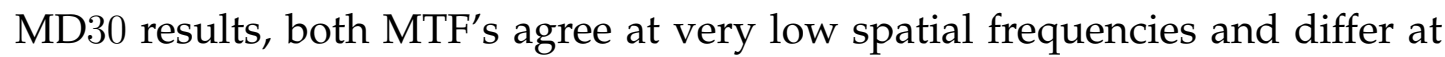
high spatial frequencies with the grid method extending to much higher spatial frequencies. The resolution at a MTF equal to 0.2 is $6.5 \mathrm{lp} / \mathrm{mm}$ for the grid method and $8.5 \mathrm{lp} / \mathrm{mm}$ for the edge method. These curves show a bimodal nature, which as explained in section 4.2 is caused by the narrow laser beam and long scattering length for a semi-transparent (weakly scattering) material. To compare the glass ceramic sample and Agfa imaging plate the MTF's are shown in figure 6.13. The glass ceramic sample shows a higher spatial resolution than the commercially available plates and is able to distinguish images at even greater spatial resolutions due to the bimodal nature of the MTF. 


\section{Chapter 7}

\section{Discussion and Conclusions}

This thesis has investigated the effect varying plate and experimental parameters has on the resolution and sensitivity of imaging plates with the use of simulations. Experiments were performed to determine the resolution of a glass ceramic and was compared to a current commercial imaging plate.

Simulations show that there are two scattering regimes where the resolution is highest, occurring at very short and very long scattering lengths, with long scattering lengths having the higher resolution. The reason behind two different regimes is due to different mechanisms limiting the spread of laser photons. For very short scattering lengths the spread of photons is limited to a small volume near the surface by strong scattering. At very long scattering lengths most of the laser photons pass straight through the plate with only a small amount being scattered and so the spatial resolution is determined by the laser beam profile. The modulation transfer functions show different forms for the two regimes which reflect the different scattering mechanisms. Large scattering lengths show a bimodal nature which at low spatial frequencies is caused by the weak scattering travelling long distances and at high spatial frequencies is due to the laser beam profile. For short scattering lengths the curve has a Gaussian-like shape which is caused by a uniform spreading of the laser photons; essentially this is a diffusion problem.

Varying the absorption is useful to limit the spread of the laser photons caused 
by scattering. This can be achieved in two ways - increasing the absorption at all wavelengths of light or by predominantly increasing the absorption of red laser light. Increasing the absorption increases the resolution as the spread of laser photons is decreased but comes at a cost of lower sensitivity. By increasing the absorption coefficient for the laser photons and keeping the absorption for PSL photons fixed, the sensitivity is greatly increased as compared to increasing the absorption for both, with similar resolution. Increasing the absorption above $\gamma=0.05 \mu \mathrm{m}^{-1}$ does not significantly change the resolution but decreases the sensitivity, as the spread of the laser photons reaches the limit set by the profile of the laser beam.

Large particle sizes show a higher resolution and lower sensitivity than small particles due to the increase in forwards scattering of the laser photons. Actual sensitivities will be greater for large particles as they have been found to show an increased PSL effect [33]. The simulation uses the Henyey-Greenstein function to model the effect particle size has on the scattering of photons which is a good approximation for particles with radius $\sim 100 \mathrm{~nm}$. For particle sizes above this, the function overestimates the contribution from forward scattering which could have an effect on the results found for a particle radius of 1 $\mu \mathrm{m}$.

Reflection from surfaces was found to be a substantive effect in the simulations. Including reflection results in laser photons travelling greater distances in the plate, decreasing the resolution. The sensitivity is increased because photons travel more than once through the plate. To reduce the effect of reflection additional index-matching layers can be added to the surfaces of the plate which will absorb the laser light and allow through the PSL light.

Glass ceramic materials typically have scattering lengths of around $1000 \mu \mathrm{m}$ for red laser light and $200 \mu \mathrm{m}$ for PSL light, but these can be changed by different thermal treatments. This puts them into the weakly scattering regime where most laser photons pass straight through and few are scattered. To 
design a high resolution glass ceramic imaging plate the material should be highly transparent (long scattering lengths) and absorption should be added to limit the range of the weak scattering tails in the LSF.

In addition to varying plate parameters, the resolution and sensitivity can be changed by experimental means. These include changing the area and intensity of the laser spot incident on the plate during read-out and changing the energy of $\mathrm{x}$-rays used to create the image.

A small laser diameter increases the resolution as it allows for the read-out of finer details. Lowering the laser power decreases the bleaching effect, leading to a higher resolution at the cost of sensitivity. Varying the $\mathrm{x}$-ray energy has very little effect on the resolution, but greatly affects the sensitivity - an increase in $\mathrm{x}$-ray energy decreases the sensitivity. Low $\mathrm{x}$-ray energies (high attenuation coefficients) are best used as more storage centres are generated in the and a higher PSL signal is achieved. However, this may be incompatible with the imaging target; usually thin aluminium ( $\sim 1 \mathrm{~mm}$ thick) is used in medical imaging to eliminate soft $x$-rays which are completely absorbed by body tissue and so do not contribute to the image but are harmful to the patient. The simulation results for x-ray energy do not include the effects of Compton scattering which will decrease the resolution especially at high energies.

The best resolution obtainable by varying experimental parameters is achieved with a small laser diameter, low laser power and a low x-ray energy.

Experimental results show that the glass ceramic materials have a higher spatial resolution than the commercial imaging plate. Two methods of obtaining the MTF for the various materials were used and the curves show slightly different results, particularly at high spatial frequencies, where the MTF curve of the grid method is higher than that for the edge method. This implies that the edge method is unable to distinguish high spatial frequencies, possibly due to the straight edge dominating over the width of the laser beam. The MTF curves agree with predictions made by the simulations; for short scattering 
lengths (Agfa plate) the curve is Gaussian-like and at large scattering lengths (glass ceramics) the curve has a bimodal nature.

The Agfa MD30 imaging plate was found to have a spatial resolution of 4.5 $\mathrm{lp} / \mathrm{mm}$ at an MTF equal to 0.2 , which is higher than the commonly accepted value of $2.5 \mathrm{lp} / \mathrm{mm}$ and nearly as large as for x-ray film $(5 \mathrm{lp} / \mathrm{mm})$. No aluminium filter was used to eliminate soft x-rays, hence, only a surface image is created on the plate which could cause the high resolution found.

The N05-02-50 glass ceramic sample was found to have a spatial resolution of $6.5 \mathrm{lp} / \mathrm{mm}$ for the edge method and $8 \mathrm{lp} / \mathrm{mm}$ for the grid method. These results are higher than for the Agfa MD30 sample but not as high as was expected, which is most likely due to the low transparency and variation in thickness of the sample. The MTF shows a bimodal nature which suggests that the sample is able to distinguish much higher resolution images than is indicated by the spatial resolution.

The model used to simulate the scattering could be expanded to include other features such as adding absorbing back layers to eliminate reflection, as is done in the commercial BaFBr plates. A more realistic phase function, or even complete Mie theory could be incorporated into the model to more accurately describe the angular dependece on scattering - especially for large particle sizes. Additional simulations could be run to investigate the effect of other variables not changed in this thesis and also to test different combinations of variables to determine the most optimal plate. Currently work is being undertaken to improve the quality of glass ceramic plates, in particular the removal of bubbles and defects. Using these techniques, glass ceramic plates could be developed with varying absorption and scattering properties to experimentally determine the highest resolution glass ceramic imaging plate.

In conclusion, glass ceramic materials have the potential to become high resolution x-ray imaging plates. Experimental and simulation results have shown that the spatial resolution of a transparent material has the ability to be greater 
than that of the current commercial crystalline imaging plates.

Simulation was found to be a valuable guide to the optimisation of glass ceramic $\mathrm{x}$-ray storage phosphor imaging plates. Using the results found by simulations the "recipe" for an ideal glass ceramic imaging plate is:

- Large scattering length (highly transparent material),

- Increased absorption of laser photons in the form of Cobalt fluoride or similar,

- Large particle sizes,

- Layers to reduce reflection from surfaces,

with optimum read-out conditions:

- Low laser power,

- Small laser diameter,

- High x-ray attenuation coefficient. 



\section{Appendix A}

This compact disc (CD) contains scattering simulation code, Mie theory code and the LabVIEW driver to run the experiment. To run the scattering simulations a text editor and a Fortran 90 compiler is required. Mie theory code can be run in MATLAB from the CD by placing the folder extension into the current directory. The experimental system is run using the Main Control VI and requires LabVIEW 8.2 as well as the included sub VI's. 



\section{References}

[1] Sonoda, M., Takano, M., Miyahara, J. \& Kato, H. Computed radiography utilizing scanning laser stimulated luminescence. Radiology 148, 833 (1983).

[2] Hayes, W. Crystals with the fluorite structure (Clarendon Press, Oxford, 1974).

[3] Edgar, A., Spaeth, J.-M., Schweizer, S., Assmann, S., Newman, P. \& MacFarlane, D. Photostimulated luminescence in a rare earth-doped fluorobromozirconate glass ceramic. Applied Physics Letters 75, 2386-2388 (1999).

[4] Qiu, J., Shimizugawa, Y., Iwabuchi, Y. \& Hirao, K. Photostimulated luminescence of $\mathrm{Ce}^{3+}$-doped alkali borate glasses. Applied Physics Letters 71, 43 (1997).

[5] Qiu, J., Shimizugawa, Y., Y, I. \& Hirao, K. Photostimulated luminescence in $\mathrm{Eu}^{2+}$-doped fluoroaluminate glasses. Applied Physics Letters 71, 759 (1997).

[6] Qiu, J., Shimizugawa, Y., Sugimoto, N. \& Hirao, K. Photostimulated luminescence in borate glasses doped with $\mathrm{Eu}^{2}$ and $\mathrm{Sm}^{3}+$ ions. Journal of Non-Crystalline Solids 222, 290 (1997).

[7] Edgar, A. (2008). Private Communication.

[8] Schweizer, S., Hobbs, L., Secu, M., Spaeth, J. M., Edgar, A. \& Williams, G. 
V. M. Photostimulated luminescence in Eu-doped fluorochlorozirconate glass ceramics. Applied Physics Letters 83, 449 (2003).

[9] Edgar, A., Williams, G. V. M., Secu, M., Schweizer, S. \& Spaeth, J. M. Optical properties of a high-efficiency glass ceramic X-ray storage phosphor. Radiation Measurements 38, 413 (2004).

[10] Halliday, D., Resnick, R. \& Walker, J. Fundamentals of Physics 6th Edition (John Wiley \& Sons, Inc., New York, 2001).

[11] Kerker, M. The scattering of light, and other electromagnetic radiation (Academic Press, New York, 1969).

[12] Bohren, C. F. \& Huffman, D. R. Absorption and scattering of light by small particles (Wiley, New York, 1983).

[13] http://www.iap.unibe.ch/publications/download/201/en/.

[14] Henyey, L. \& Greenstein, J. Diffuse Radiation in the Galaxy. Astrophysical Journal 93 (1941).

[15] Griffiths, D. J. Introduction to Electrodynamics (Prentice Hall, Upper Saddler River, NJ, 1999).

[16] Schweizer, S. Physics and Current Understanding of X-Ray Storage Phosphors. Physica Status Solidi (a) 187, 335 (2001).

[17] Thoms, M. Image properties of polycrystalline storage films. Applied Optics 35, 3702 (1996).

[18] Fasbender, R., Li, H. \& Winnacker, A. Monte Carlo modeling of storage phosphor plate readouts. Nuclear Instruments and Methods in Physics Research Section A: Accelerators, Spectrometers, Detectors and Associated Equipment 512, 610 (2003). 
[19] Edgar, A., Williams, G. V. M., Schweizer, S. \& Spaeth, J. M. Spatial resolution of a glass-ceramic X-ray storage phosphor. Current Applied Physics 6, 399 (2006).

[20] Chen, G., Johnson, J., Weber, R., Nishikawa, R., Schweizer, S., Newman, P. \& MacFarlance, D. Fluorozirconate-based nanophase glass ceramics for high-resolution medical x-ray imaging. Journal of Non-Crystalline Solids 352,610 (2006).

[21] Schweizer, S. \& Johnson, J. A. Fluorozirconate-based glass ceramic X-ray detectors for digital radiography. Radiation Measurements 42, 632 (2007).

[22] Press, W. H., Flannery, B. P., Teukolsky, S. A. \& Vetterling, W. T. Numerical Recipes in Fortran 90: The Art of Parallel Scientific Computing, vol. 2 (Cambridge University Press, Cambridge, 1996).

[23] Hendy, S. \& Schebarchov, D. (2007). Private Communication.

[24] http://gcc.gnu.org/wiki/gfortran.

[25] http:/ / en.wikipedia.org/wiki/euler_angles.

[26] Suzuki, Y., Sibley, W. A., El Bayoumi, O. H., Roberts, T. M. \& Bendow, B. Optical properties of transition-metal ions in zirconium-based metal fluoride glasses and $\mathrm{MgF}_{2}$ crystals. Physical Review B 35, 4472 (1987).

[27] http:/ / en.wikipedia.org/wiki/monochromator.

[28] Pedrotti, F. L. \& Pedrotti, L. S. Introduction to Optics (Prentice-Hall, Inc., New Jersey, 1993), second edn.

[29] Ohnishi, A., Mori, N. \& Kan'no, K. Thermal Stability of Photostimulated Luminescence Centers in $\mathrm{BaFBr}$ and $\mathrm{BaFBr}: \mathrm{Eu}^{2+}$ Single Crystals. Japanese Journal of Applied Physics 38, 6782 (1999). 
[30] http://www.agfa.com/en/he/products_services/all_products/cr md40_general_plate.jsp.

[31] Aggarwal, I. D. \& Lu, G. Fluoride Glass Fiber Optics (Academic Press, Inc., San Diego, 1991).

[32] Klug, H. P. \& Alexander, L. E. X-ray Diffraction Procedures for Polycrystalline and Amorphous Materials (John Wiley \& Sons, New York, 1974), second edn.

[33] Schweizer, S., Hobbs, L. W., Secu, M., Spaeth, J.-M., Edgar, A., Williams, G. V. M. \& Hamlin, J. Photostimulated luminescence from fluorochlorozirconate glass ceramics and the effect of crystallite size. Journal of Applied Physics 97, 083522 (2005). 